\title{
A Newtonian Approach to Predict the Parameters Required to Machine High Aspect Ratio Channels of Prescribed Topography
}

by

\begin{abstract}
Aria Ghazavi
Bachelor of Mechanical Engineering, Ryerson University, Toronto, Canada 2016
\end{abstract}
A thesis presented to Ryerson University
in partial fulfillment of the requirements for the degree of
Master of Applied Science in the program of
Mechanical and Industrial Engineering

Toronto, Ontario, Canada, 2018

(C) Aria Ghazavi, 2018 


\section{Author's Declaration for Electronic Submission of Thesis}

I hereby declare that I am the sole author of this dissertation. This is a true copy of the dissertation, including any required final revisions, as accepted by my examiners.

I authorize Ryerson University to lend this dissertation to other institutions or individuals for the purpose of scholarly research.

I further authorize Ryerson University to reproduce this dissertation by photocopying or by other means, in total or in part, at the request of other institutions or individuals for the purpose of scholarly research.

I understand that my dissertation may be made electronically available to the public. 


\title{
Abstract
}

\section{A Newtonian Approach to Predict the Parameters Required to Machine High Aspect Ratio Channels of Prescribed Topography}

\author{
Aria Ghazavi \\ Master of Applied Science, 2018 \\ Mechanical and Industrial Engineering \\ Ryerson University
}

Control of the microchannels' cross-sectional shape may be of interest in micro-heat sinks, microfluidic particle sorting, and micro-machine lubrication applications. Previously, inverse methods have been used to determine the abrasive jet micromachining (AJM) traverse speed and path required to sculpt the desired cross-section for low Aspect Ratio (AR, the ratio of depth to width, see page xiv) topographies $(<0.06)$. This thesis introduces an iterative inverse method which allows prediction of the machining procedure required to sculpt high AR (>0.06-1) microchannels of prescribed cross-sectional shape using mask-less AJM. The predictions were experimentally verified for trapezoidal and semi-circular micro-channels and protruded features in borosilicate glass, and symmetric and non-symmetric wedges in poly-methyl-methacrylate (PMMA). Overall, the average accuracy of the machined profiles was $93.6 \%$ in borosilicate glass and $91 \%$ in PMMA. The methodology opens up new possibilities for the micro-fabrication of high-aspectratio micro-features of virtually any desired shape. 


\section{Acknowledgments}

I would like to thank:

* My supervisor and mentor, Dr. Marcello Papini, for his untiring guidance and tolerance helping me navigate my way in choosing an impactful project and presenting it.

* The Natural Sciences and Engineering Research Council (NSERC) for supporting and funding this project.

* Dr. Reza Sookhak Lari for his guidance in applying optimization to shallow ARs and his method of identifying edges of channels.

* Dr. Ali Nouhi for commenting on various aspects of the project and recommending resources as well as troubleshooting the predictions and helping with interpretation of the results and centering profiles.

* Ryerson University for housing the laboratory and the equipment used for the experiments.

Mr. Chao Ma for maintaining the equipment used to conduct these experiments.

My Father Mr. Hossein Ghazavi and my mother, Dr. Shadi Babazadeh, for their unceasing support through all the tumultuous moments of my studies. 


\section{Dedication}

I would like to dedicate this dissertation to all the students of Mechanical Engineering who

pursue their quest for knowledge and explanation of the outside world, and use that knowledge to improve others' lives. 


\section{Table of Contents:}

AUTHOR's DECLARATION FOR ELECTRONIC SUBMISSION OF THESIS ........................................ ii

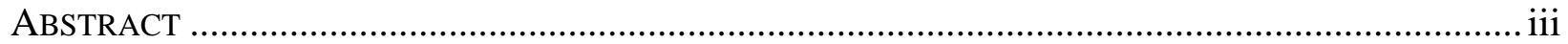

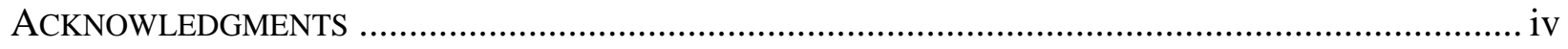

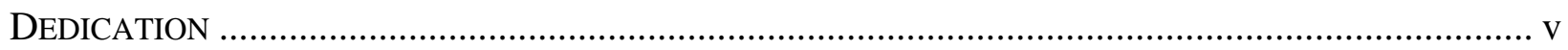

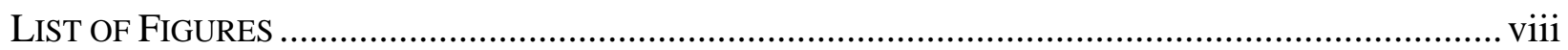

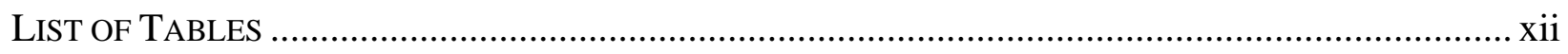

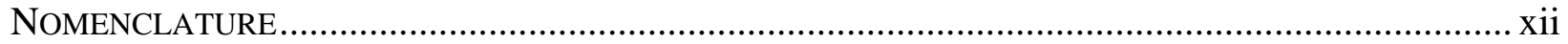

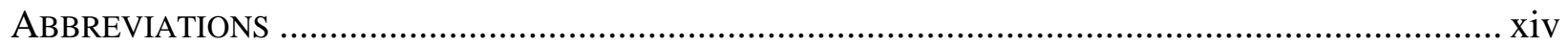

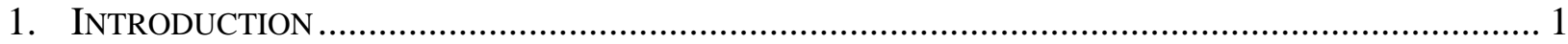

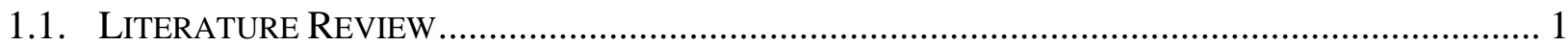

1.1.1. Application of Microchannels and Features Created with AJM..................................... 1

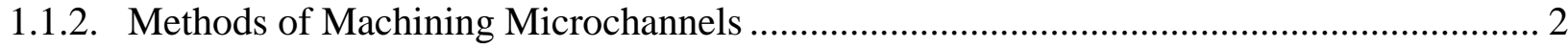

1.1.3. Benefits of using AJM and the Parameters that Influence it ........................................ 2

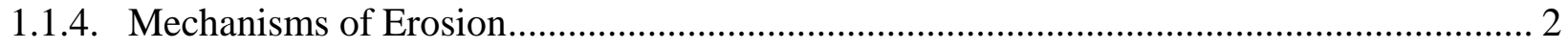

1.1.5. Surface Quality of Channels Machined in AJM .......................................................... 3

1.1.6. Application of Masks in AJM ............................................................................... 3

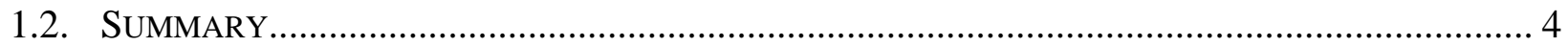

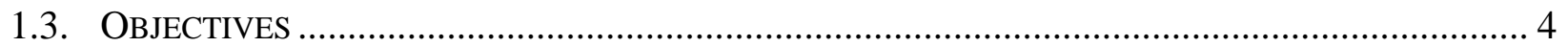

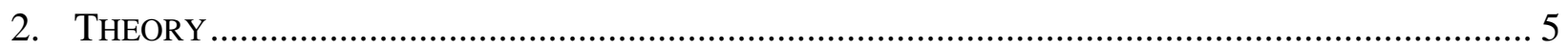

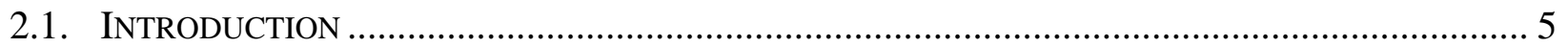

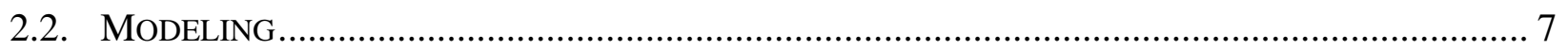

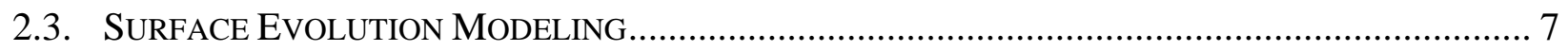

2.4. DEVIATION FROM LiNEAR SURFACE EVOLUTION........................................................... 10

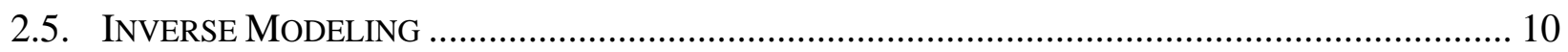

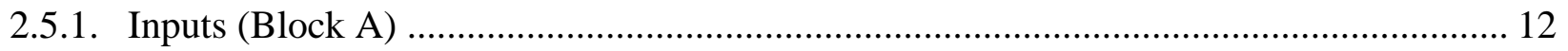

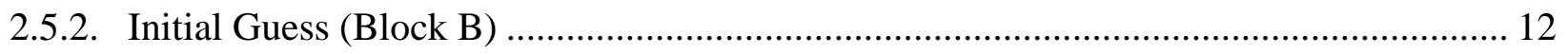

2.5.3. Propagate Surface Based on Current Guess (Block C) .............................................. 12

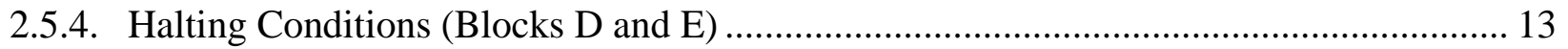

2.5.5. Adjustment of Scaled Erosive Efficacy Each Iteration (Block F) ............................... 13

2.6. GENERATION OF REQUIRED EROSIVE EFFICACY USING AdJACENT PASSES......................... 14

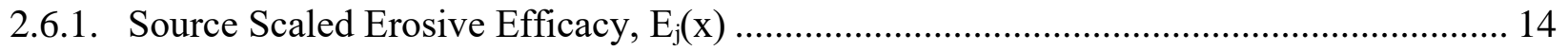

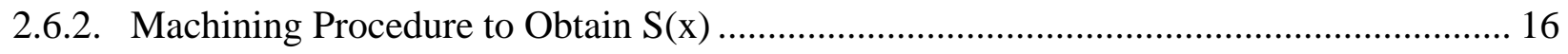

2.7. MEASURING THE RAdiUS OF CURVATURE AND IDENTIFYING THE EDGES.......................... 16

2.8. ROUNDING OF EDGES............................................................................................ 18

2.9. UNIQUENESS AND ROBUSTNESS OF THE INVERSE SOLUTION ....................................... 19

2.9.1. Predicting a Feature in $\mathrm{E}_{\mathrm{r}}$ Disappeared through Multiple Repeats ................................ 19

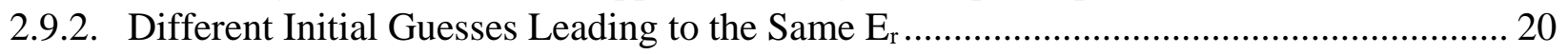

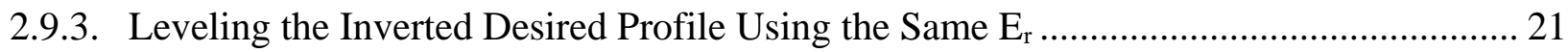

2.10. Direct Application of Optimization to High A.R. Channels................................ 22

2.11. Inherent Tendency to Form Wedge-Shaped Profiles in Brittle Materials......... 24 


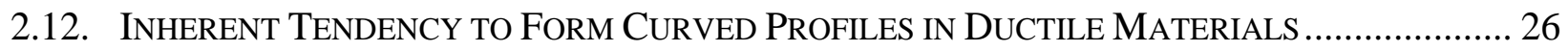

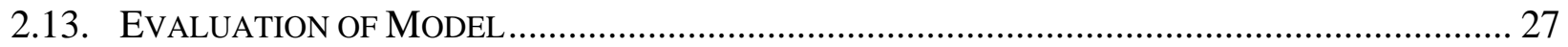

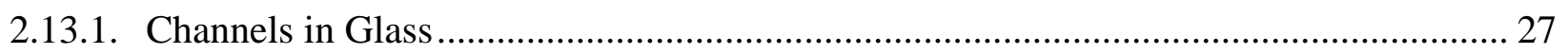

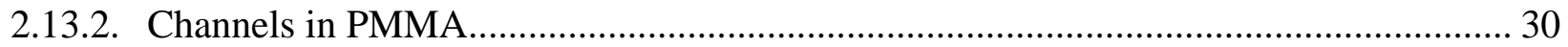

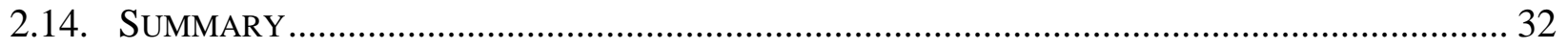

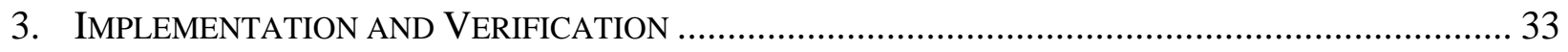

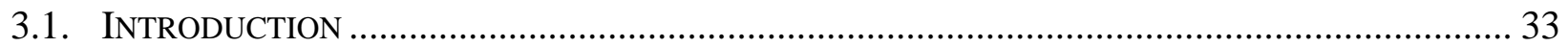

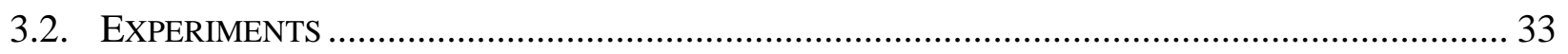

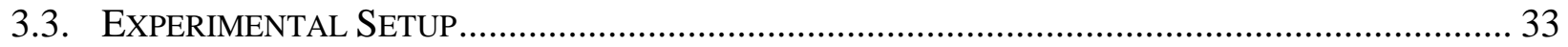

3.4. MACHINING OF CHANNELS IN GLASS AND PMMA ......................................................... 34

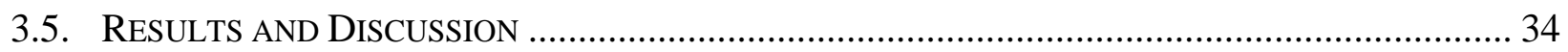

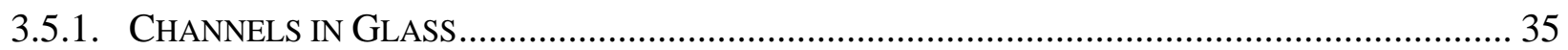

3.5.1.1. Rectangular - Limitation of Maximum Sidewall Slope ........................................... 35

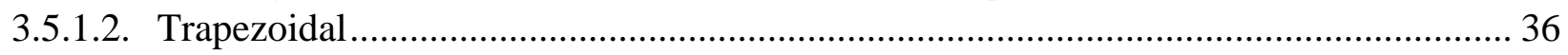

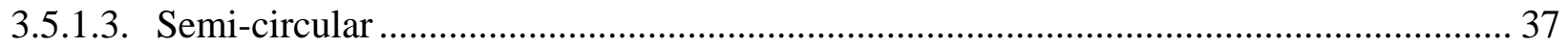

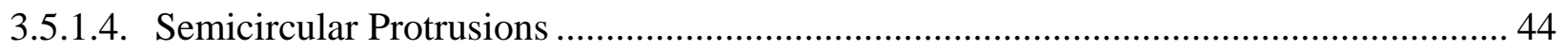

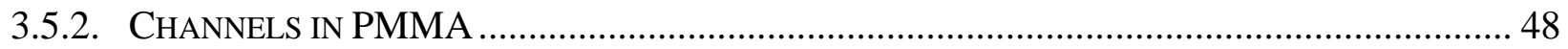

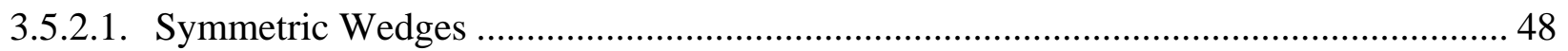

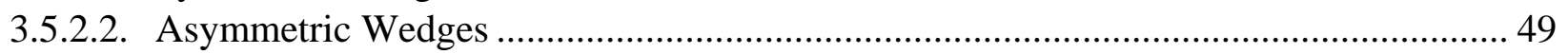

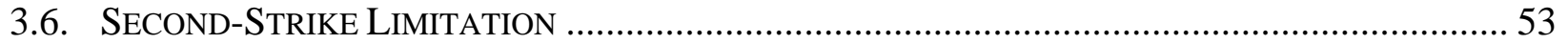

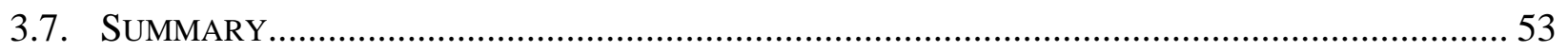

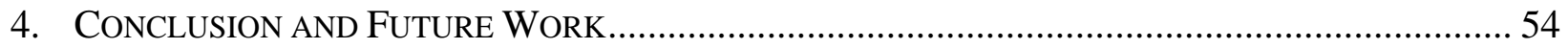

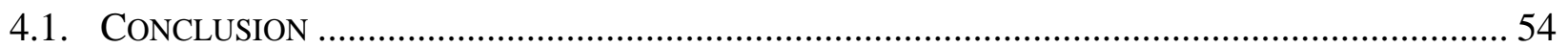

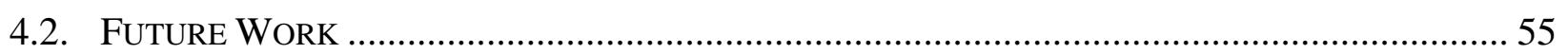

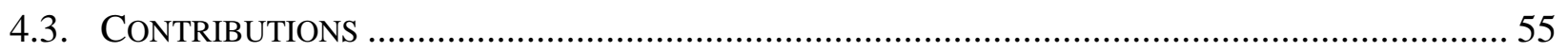

A.1. TABLE OF MACHINING PARAMETERS FOR CHANNELS IN GLASS ....................................... 57

A.2. TABlE OF MACHINING PARAMETERS FOR CHANNELS IN PMMA ........................................ 59

A.3. SECOND-STRIKE CALCULATIONS BASED ON RAY-TRACING AND RIGID BODY DYNAMICS ..... 60

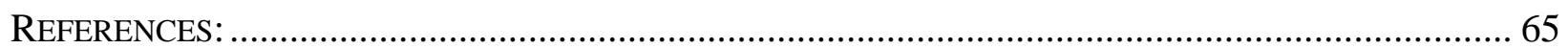




\section{List of Figures:}

Figure 2.3.1: Summary of the inverse method to determine the scaled erosive efficacy required to machine the desired surface shape. 9

Figure 2.4.1: Average absolute percentage difference between predicted profiles of a singlesource straight channel evolving to a given aspect ratio when assuming a linearly scaled surface evolution, and its evolution according to equations 2-3 and 2-4.

Figure 2.5.1:(a) Schematic drawing of multiple adjacent passes of the source (nozzle) needed to produce a channel profile of prescribed shape and (b) the scaled erosive efficacies of two adjacent passes 1 and 2 which superimpose to yield the deeper profile. The evolved surface resulting from multiple repetitions of the adjacent passes is also shown.

Figure 2.6.1: (a) Source's scaled erosive efficacy and average source strengths on the glass and (b) PMMA, respectively (c) The curve fitted data of the average scaled erosive efficacy extracted at various scan speeds for both glass

Figure 2.7.1: Radius of curvature for the single source evolution and the shift in extrema with increasing number of passes, solid line depicting the radius of curvature of the first pass at each location, the dotted line showing that of the second pass's profile, dashed line that of the third pass, dashed-dotted line that of the fourth and dashed-double-dotted that of the fifth pass's profile

Figure 2.7.2: Half of the profile extracted from a channel eroded in the glass with nozzle scanning the specimen with $1 \mathrm{~mm} / \mathrm{s}$. Lines passing through consequent points of inflection along the profile in each repeat were drawn, proving the profile approaching a line

Figure 2.8.1: (a) Quasi-rectangular scaled erosive efficacies, and (b) minimum radius of curvature across the profile resulting from evolving the efficacy using Equation 2-3. 18 Figure 2.9.1: (a) The original (solid line) and required (dashed line) normalized scaled erosive efficacies scaled by the number of repetitions predicted to evolve to the V-shaped target. (b) The predicted surface after using $\mathrm{E}_{0}(\mathrm{x})$ in Equation 2-3. after $\mathrm{n}_{\mathrm{f}}=50$ repeats, evolved under the original $\mathrm{E}_{\mathrm{r}}$ (solid line) and the predicted $\mathrm{E}_{\mathrm{r}}$ (dashed line)....

Figure 2.9.2: (a) Different initial guesses (the dashed line represents a rectangular pocket with rounded edges, the dotted line a sinusoidal curve and the solid line the linearly scaled target) used as inputs for the inverse algorithm; (b) The predicted required scaled erosive efficacies $E_{\mathrm{r}}$ (x) scaled by the number of repeats and divided by the target's maximum depth (the dashed, dotted and solid line correspond to rectangular, sinusoidal and linearly scaled initial guesses seen in (a)).....

Figure 2.9.3: (a) evolution of a brittle surface (glass) given the dotted profile as erosive efficacy (b) erosion of a protrusion of mirrored topography given the desired mirror profile in solid line, the assumed initial surface with dashed line and the final eroded surface after $\mathrm{n}_{\mathrm{f}}$ repeats with dotted line machined in glass.

Figure 2.9.4: (a) comparison between the evolved surface of the mirrored profile in dashed line and the predicted erosive efficacy mirrored about the zero line of the surface shown with a solid line. (b) The location of the leveled bump after $n_{f}+1$ number of repeats given the same scaled erosive efficacy . 
Figure 2.10.1: Comparison between the experimental results (markers $\times$ for $16 \mathrm{~mm} / \mathrm{s}, \triangle$ for 8 $\mathrm{mm} / \mathrm{s}, \diamond$ for $2 \mathrm{~mm} / \mathrm{s}$ and $\square$ for $1 \mathrm{~mm} / \mathrm{s}$ ) and surface evolution predictions by MOL (shown with the dashed lines) and FD (shown with the dotted lines) ..................................................... 23 Figure 2.11.1: (a) The required erosive efficacy $E_{i}(x)$ predicted by the inverse algorithm after $i$ iterations (i=10 represented by the triangular markers $(\triangle), i=50$ shown by the diamond markers $(\diamond)$ and $\mathrm{i}=100$ iterations shown by the square markers $(\square)$ ) required to create (b) a particular desired $\mathrm{D}(\mathrm{x})$ asymmetric wedge in glass, together with the evolved surfaces using $\mathrm{E}_{\mathrm{i}}(\mathrm{x}) \ldots \ldots \ldots . .25$ Figure 2.12.1: (a) The scaled erosive efficacy $\mathrm{E}_{\mathrm{i}}(\mathrm{x})$ predicted by the inverse algorithm after $\mathrm{i}$ iterations (i=10 represented by the triangular markers $(\triangle), i=50$ shown by the diamond markers $(\diamond)$ and $\mathrm{i}=100$ iterations shown by the square markers $(\square)$ ) required to create $(\mathrm{b})$ a desired $\mathrm{D}(\mathrm{x})$ $500 \mu \mathrm{m}$ radius circular cross-section in PMMA, together with the evolved surfaces using $\mathrm{E}_{\mathrm{i}}(\mathrm{x}) .26$ Figure 3.5.1: Dependence of erosion rate on the angle of attack for glass (dashed line) [64] and PMMA (solid line) [35] described by $\mathrm{g}(\alpha)$ as discussed in chapter 2 35 Figure 3.5.1.1: (a) Predicted scaled erosive efficacy, $\mathrm{E}_{\mathrm{r}}(\mathrm{x})$, required to produce (b) desired, $\mathrm{D}(\mathrm{x})$, rectangular microchannel, with predicted final profile, $\xi(\mathrm{x})$. 35 Figure 3.5.1.2: (a) Predicted $E_{r}(x)$ shown with the solid line, and approximate $S(x)$ (shown with the $\diamond$ markers) scaled erosive efficacy required to produce (b) desired, $\mathrm{D}(\mathrm{x})$ (solid line), rectangular microchannel, with predicted, $\xi(\mathrm{x})$ (dashed line) and measured final profiles, displayed with $\diamond$ in (b). The predicted surface and the desired surface were so close that they

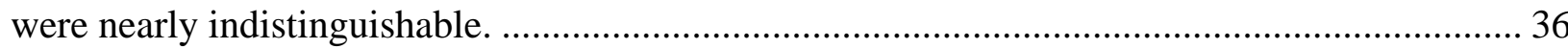

Figure 3.5.1.3: SEM of trapezoidal cross-section machined in the glass. ................................. 37 Figure 3.5.1.4: (a) Predicted $\mathrm{E}_{\mathrm{r}}(\mathrm{x})$ (solid line), and approximate $\mathrm{S}(\mathrm{x})$ ( $\diamond$ markers) scaled erosive efficacy required to produce (b) desired, D(x) (solid line), $500 \mu \mathrm{m}$ radius semi-circular channel, with predicted, $\xi(\mathrm{x})$ (dashed line) and measured final profiles ( $\diamond$ markers). Five sources were used to create the profile. Only half the symmetric channel is shown. ................................... 38 Figure 3.5.1.5: (a) Predicted $\mathrm{E}_{\mathrm{r}}(\mathrm{x})$ (solid line), and approximate $\mathrm{S}(\mathrm{x})$ (dashed line) superimposed scaled erosive efficacy required to produce (b) desired, D(x) (solid line), $600 \mu \mathrm{m}$ radius semicircular channel, with predicted, $\xi(\mathrm{x})$ (dashed line) and measured final profiles ( $\diamond$ markers). Nine sources were used to create the profile. Only half the symmetric channel is shown......... 39 Figure 3.5.1.6: (a) Predicted $\mathrm{E}_{\mathrm{r}}(\mathrm{x})$ (solid line), and approximate $\mathrm{S}(\mathrm{x})$ ( $\diamond$ markers) scaled erosive efficacy required to produce (b) desired, D(x) (solid line), $800 \mu \mathrm{m}$ radius semi-circular channel, with predicted, $\xi(\mathrm{x})$ (dashed line) and measured final profiles ( $\diamond$ markers). Ten sources were used to create the profile. Only half the symmetric channel is shown.

Figure 3.5.1.7: (a) Predicted $\mathrm{E}_{\mathrm{r}}(\mathrm{x})$ (solid line), and approximate $\mathrm{S}_{1}(\mathrm{x})$ ( $\diamond$ markers) superimposed scaled erosive efficacy created using 10 sources and $\mathrm{S}_{2}(\mathrm{x})(\times$ markers), using 8 sources required to produce desired, $\mathrm{D}(\mathrm{x})$ (solid line), $700 \mu \mathrm{m}$ radius semi-circular channel, with predicted, $\xi(\mathrm{x})$ (dashed line) and measured final profiles ( $\diamond$ markers) using (b) ten and (c) eight sources. Only half the symmetric channels are shown.

Figure 3.5.1.8: (a) Predicted $\mathrm{E}_{\mathrm{r}}(\mathrm{x})$ (solid line), and approximate $\mathrm{S}_{1}(\mathrm{x})(\diamond$ markers) superimposed scaled erosive efficacy created using 10 sources and $\mathrm{S}_{2}(\mathrm{x})(\times$ markers), using 8 sources required to produce desired, $\mathrm{D}(\mathrm{x})$ (solid line), $900 \mu \mathrm{m}$ radius semi-circular channel, with predicted, $\xi(\mathrm{x})$ 
(solid line) and measured final profiles ( $\diamond$ markers) using (b) 10 and (c) 8 sources. Only half the symmetric channels are shown.

Figure 3.5.1.9: SEM of semicircular cross section channel (radius $=800 \mu \mathrm{m}$ ) machined in the glass the scaled erosive efficacy of which could be described in section 3.3 ................................. 44 Figure 3.5.1.10: (a) Predicted $\mathrm{E}_{\mathrm{r}}(\mathrm{x})$ (solid line), and approximate $\mathrm{S}(\mathrm{x})(\diamond$ markers) superimposed scaled erosive efficacy required to produce (b) desired, $\mathrm{D}(\mathrm{x})$ (solid line), $600 \mu \mathrm{m}$ radius semicircular protrusion in the channel, with predicted, $\xi(\mathrm{x})$ (dashed line) and measured final profiles ( $\diamond$ markers). 22 sources were used to create the profile. Only half the symmetric channel is shown.

Figure 3.5.1.11: (a) Predicted $\mathrm{E}_{\mathrm{r}}(\mathrm{x})$ (solid line), and approximate $\mathrm{S}(\mathrm{x})(\diamond$ markers) superimposed scaled erosive efficacy required to produce (b) desired, D(x) (solid line), $800 \mu \mathrm{m}$ radius semicircular protrusion in the channel, with predicted, $\xi(\mathrm{x})$ (dashed line) and measured final profiles ( $\diamond$ markers). 26 sources were used to create the profile. Only half the symmetric channel is shown.

Figure 3.5.1.12: (a) Predicted $\mathrm{E}_{\mathrm{r}}(\mathrm{x})$ (solid line), and approximate $\mathrm{S}(\mathrm{x})(\diamond$ markers) scaled erosive efficacy required to produce (b) desired, $\mathrm{D}(\mathrm{x})$ (solid line), $600 \mu \mathrm{m}$ radius semi-circular protrusion in the channel, with predicted, $\xi(\mathrm{x})$ (dashed line) and measured final profiles ( $\diamond$ markers). 22 sources were used to create the profile. Only half the symmetric channel is shown.

Figure 3.5.2.1: (a) Predicted $\mathrm{E}_{\mathrm{r}}(\mathrm{x})$ (solid line), and approximate $\mathrm{S}(\mathrm{x})(\diamond$ markers) scaled erosive efficacy required to produce (b) desired, D(x) (solid line), symmetric wedge with predicted, $\xi(\mathrm{x})$ (dashed line) and measured final profiles ( $\diamond$ markers). Only half the symmetric channel is

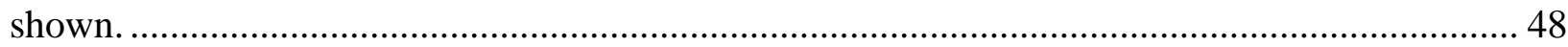

Figure 3.5.2.2: A channel with a wedge-shaped cross-section machined in PMMA 49 Figure 3.5.2.3: (a) Predicted $\mathrm{E}_{\mathrm{r}}(\mathrm{x})$ (solid line), and approximate $\mathrm{S}(\mathrm{x})(\diamond$ markers) scaled erosive efficacy required to produce (b) desired, D(x) (solid line), $20^{\circ}$ asymmetric wedges with predicted, $\xi(\mathrm{x})$ (dashed line) and measured final profiles ( $\diamond$ markers). 50 Figure 3.5.2.4: (a) Predicted $\mathrm{E}_{\mathrm{r}}(\mathrm{x})$ (solid line), and approximate $\mathrm{S}(\mathrm{x})$ ( $\diamond$ markers) scaled erosive efficacy required to produce (b) desired, $\mathrm{D}(\mathrm{x})$ (solid line), $20^{\circ}$ asymmetric wedges with predicted, $\xi(\mathrm{x})$ (dashed line) and measured final profiles ( $\diamond$ markers). 51 Figure 3.5.2.5: The asymmetric wedges cross-section with a $30^{\circ}$ incline machined in PMMA. Note, the articles at the right side of the channel were created in the cutting process as burs and were not the result of AJM machining.

Figure 3.5.2.6: (a) Predicted $\mathrm{E}_{\mathrm{r}}(\mathrm{x})$ (solid line), and approximate $\mathrm{S}(\mathrm{x})$ ( $\diamond$ markers) scaled erosive efficacy required to produce (b) desired, $\mathrm{D}(\mathrm{x})$ (solid line), $45^{\circ}$ asymmetric wedges with predicted, $\xi(\mathrm{x})$ (dashed line) and measured final profiles ( $\diamond$ markers).

Figure A.1: Schematics of a ricocheting particle with the angles of interest with the dashed arrows showing the trajectory of points on the sphere. 59

Figure A.2: The algorithm used to identify the location of second-strike 


\section{List of Tables:}

Table 2-1: Column 1: the predicted required scaled erosive efficacy $\left(\mathrm{E}_{\mathrm{r}}(\mathrm{x})\right)$ for dashed line, the GRG-nonlinear's $\mathrm{S}(\mathrm{x})$, dotted line, inverse compared to the initial guess $\left(\mathrm{E}_{0}(\mathrm{x})\right)$ given by Equation 2-6, solid line; column 3: the evolved $\xi(\mathrm{x}, \mathrm{n})$ from repeating $\mathrm{E}_{\mathrm{r}}(\mathrm{x})$ for dashed line, from repeating GRG-nonlinear's $\mathrm{S}(\mathrm{x})$, dotted line, compared to $\mathrm{D}(\mathrm{x})$, by a solid line and cross markers

Table 2-2: Column 1: the predicted required scaled erosive efficacy $\left(\mathrm{E}_{\mathrm{r}}(\mathrm{x})\right)$ for dotted line, inverse compared to the initial guess $\left(\mathrm{E}_{0}(\mathrm{x})\right)$ given by Equation 2-4, solid line; column 3: the evolved $\xi(\mathrm{x}, \mathrm{n})$ from repeating $\mathrm{E}_{\mathrm{r}}(\mathrm{x})$ for dotted line, compared to $\mathrm{D}(\mathrm{x})$, by a solid line and cross

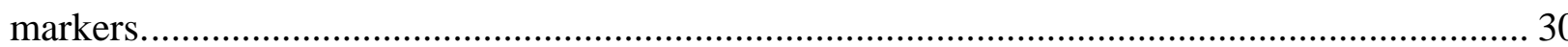

Table A-1: The machining parameters for creating the semicircular features in the brittle glass 56 Table A-2: The machining parameters and the algorithm iterations for creating the semicircular protrusions in the brittle glass

Table A-3: The machining parameters and the iterations through the inverse algorithm used to machine the specified channels in PMMA 


\section{Nomenclature}

$x$

$t$

$\xi(x, t)$

$k$

$\epsilon$

$E(x)$

$n$

$\zeta_{1}$

$h$

$\zeta *_{1}$

$T$

$t^{*}$

$\partial t / \partial n$

$E_{s}(x)$

$E_{j}(x)$

$k_{1}$

$k_{2}$

$H_{v}$

$E_{r}(x)$

$n_{f}$

$D(x)$

Tol

$E_{0}(x)$

no

do

$i$

$E_{i}(x)$

$n_{i}$

$\xi_{i}\left(x, n_{i}\right)$

$i_{\text {max }}$

$d_{i}$

$\delta$

$K_{s}$

$v_{s}$

$a_{\gamma}$

$b_{\gamma}$

$\omega_{0}$

W

$\Pi(x / W)$

$S$

$\kappa$

$\left(x_{0}\right)_{\kappa}$
Lateral location along the channel's cross-section $(\mu \mathrm{m})$

Time spent by the nozzle dwelling over each $x(\mathrm{~s})$

Instantaneous depth of the evolving surface

Normal velocity's exponent for brittle erosion

Viscous smoothing factor

Erosive efficacy $(\mu \mathrm{m} / \mathrm{s})$

Number of repeats

Maximum depth of the channel after the first repeat $(\mu \mathrm{m})$

Nozzle's height off-set from the target's surface $(\mu \mathrm{m})$

Normalized maximum depth of the channel

Nozzle's characteristic time (s)

Normalized time

Time required to complete one repeat (s)

Scaled erosive efficacy $(\mu \mathrm{m})$

Source scaled erosive efficacy $(\mu \mathrm{m})$

First experimental exponent affected by the impact condition

Second experimental exponent affected by the impact condition

Magnitude of the Vicker's Hardness of the target material

Required erosive efficacy $(\mu \mathrm{m})$

Final number of repeats

Desired profile $(\mu \mathrm{m})$

Tolerance for depth deviation from the desired profile $(\mu \mathrm{m})$

Initial guess for the scaled erosive efficacy $(\mu \mathrm{m})$

Initial guess for the number of repeats

Initial guess for the ratio of depths

Number of iterations through the algorithm

Predicted scaled erosive efficacy at the $i$ th iteration $(\mu \mathrm{m})$

Predicted number of repeats at the $i$ th iteration to machine $D(x)$

Evolving surface at the $i$ th iteration after $n_{i}$ repeats $(\mu \mathrm{m})$

Maximum number of algorithm's iterations

Ratio of depths at the center

Depth adjustment correction

Source strength $\left(\mu \mathrm{m}^{2} / \mathrm{s}^{2}\right)$

Nozzle's scan speed $(\mathrm{mm} / \mathrm{s})$

Fourier coefficients, cos multiplicandes

Fourier coefficients, sin multiplicandes

Fourier frequency $(1 / \mu \mathrm{m})$

Width of the desired channel $(\mu \mathrm{m})$

Box function effective between the two edges of the desired profile

Approximated scaled erosive efficacy $(\mu \mathrm{m})$

Nozzle/source index

Lateral location of the $\kappa$ th nozzle/source centerline $(\mu \mathrm{m})$ 


\begin{tabular}{|c|c|}
\hline$\left(v_{s}\right)_{\kappa}$ & Scan speed of the $\kappa$ th nozzle/source scan speed $(\mathrm{mm} / \mathrm{s})$ \\
\hline$\left(E_{j}\right)_{\kappa}(x)$ & $\kappa$ th source's scaled erosive efficacy contribution $(\mu \mathrm{m})$ \\
\hline$m$ & Maximum index for an adjacent pass \\
\hline$R(x)$ & Shallow profiles' objective function $(\mu \mathrm{m})$ \\
\hline$E_{r 1}$ & Scaled erosive efficacy required to machine a recessive profile $(\mu \mathrm{m})$ \\
\hline$E_{r_{2}}$ & Scaled erosive efficacy required to machine a protruding profile $(\mu \mathrm{m})$ \\
\hline$\delta n$ & Infinitesimal change in (differential of) the number of repeats \\
\hline$\Delta n$ & Size of the mesh used for number of repeats in FD \\
\hline$\Delta x$ & Size of the mesh used for lateral space in FD $(\mu \mathrm{m})$ \\
\hline$R_{\chi}$ & Objective function for direct application of GRG-nonlinear \\
\hline$l$ & Index of the $\kappa$ th source's mirrored counterpart \\
\hline$\left(x_{0}\right)_{l}$ & Lateral location of the mirrored equivalent of the $\kappa$ th source $(\mu \mathrm{m})$ \\
\hline$x_{0}$ & Lateral location of primary impact of a ricocheting particle $(\mu \mathrm{m})$ \\
\hline$r_{p}$ & Radius of the particle $(\mu \mathrm{m})$ \\
\hline$\alpha$ & Angle of tangent to the surface at the point of first strike with the horizon \\
\hline B & Angle of tangent to the surface at the point of second-strike with the horizon \\
\hline$e$ & Coefficient of restitution \\
\hline$\mu$ & Impulse ratio/ coefficient of Coulomb friction for dry friction \\
\hline$\mu_{c}$ & Critical impulse ratio \\
\hline$v_{t}$ & Initial tangential velocity component $(\mathrm{m} / \mathrm{s})$ \\
\hline$v_{n}$ & Initial normal velocity component (m/s) \\
\hline$V_{n}$ & Final normal velocity component at the point of contact $(\mathrm{m} / \mathrm{s})$ \\
\hline$V_{t}$ & Final tangential velocity component at the point of contact $(\mathrm{m} / \mathrm{s})$ \\
\hline$\omega$ & Initial angular speed $(1 / \mathrm{s})$ \\
\hline$\Omega$ & Final angular speed $(1 / s)$ \\
\hline$k_{G}$ & Particle's gyration radius about its center of mass $(\mu \mathrm{m})$ \\
\hline A & Angle of the second trajectory projected from COM with the horizon \\
\hline $\bar{V}_{n}$ & Normal component of COM's velocity after the first strike $(\mathrm{m} / \mathrm{s})$ \\
\hline $\bar{V}_{t}$ & Tangential component of COM's velocity after the first strike (m/s) \\
\hline$x_{2 n d}$ & Location of COM at second-strike $(\mu \mathrm{m})$ \\
\hline $\bar{V}$ & COM's speed after the first strike (mm/s) \\
\hline$\Lambda$ & Ratio of COM's normal over tangential velocity component \\
\hline$v\left(x_{0}\right)$ & Speed of the vertically projected particle before impact $(\mathrm{m} / \mathrm{s})$ \\
\hline E2nd & Oblique contribution to the scaled erosive efficacy at second-strike $(\mu \mathrm{m})$ \\
\hline$R_{c}$ & Radius of the target semi-circle $(\mu \mathrm{m})$ \\
\hline$W_{s}$ & Width of an individual source $(\mu \mathrm{m})$ \\
\hline
\end{tabular}




\section{Abbreviations}

$\begin{array}{ll}\text { AJM } & \text { Abrasive Jet Machining } \\ \text { ASJM } & \text { Abrasive Slurry Jet Machining } \\ \text { MEMS } & \text { Micro Electro-Mechanic Systems } \\ \text { EDM } & \text { Electric Discharge Machining } \\ \text { AIDS } & \text { Acquired Immune Deficiency Syndrome } \\ \text { LASER } & \text { Light Amplification by Stimulated Emission of Radiation } \\ \text { PDMS } & \text { Polydimethylsiloxane } \\ \text { A.R. } & \text { Aspect Ratio } \\ \text { PDE } & \text { Partial Differential Equation } \\ \text { MOL } & \text { Method of Lines } \\ \text { PMMA } & \text { Poly(Methyl-Methacrylate) } \\ \text { FD } & \text { Finite Difference } \\ \text { GRG } & \text { General Reduced Gradient } \\ \text { AWJM } & \text { Abrasive Water Jet Machining } \\ \text { COM } & \text { Center of Mass }\end{array}$




\section{Introduction}

Abrasive jet machining (AJM) is a nontraditional technology which uses microscale particles accelerated by pressurized air to remove material from a target material. The multiple particle collisions can cause lateral cracks if the target is brittle, shear, cut and plough the material if it is ductile.

The main areas of application of AJM have been to create micro-channels employed in microfluidic devices, optic elements, micro heat sinks, and Micro-Electromechanical Systems (MEMS) [1] [2]. Compared to other machining methods which could be used to create such features, e.g. electric discharge machining (EDM)[3], chemical etching and laser machining, AJM does not leave excessive electric charges on the machined product or damage electronically sensitive elements that may be connected to the target's surface, it does not chemically contaminate or oxidize the surface nor does it leave a significant heat affected zone, and is relatively cost effective[4].

Despite its advantages, using AJM has proven challenging in the past as the mechanism through which bombardment of particles erode the surface of the target and the location of the surface they leave could only be predicted by methods that require extensive computational power and mathematical prowess. This, in turn, has complicated and limited the ability to control the process to machine specifically shaped features.

In this regard, recent efforts have used a superposition of adjacent passes to control the sidewall slope of the channels created by AJM and machine features at very low aspect ratios[5] [6]. This thesis extends the application of adjacent pass AJM machining to high aspect ratios by introducing an inverse algorithm which predicts the required distribution of the scaled erosive efficacy (defined in section 2.3) that after multiple repetitions will erode the surface of the target to the desired shape.

\subsection{Literature Review}

The following section presents a general overview of the growing applications of AJM, its benefits compared to other micromachining procedures, solid particle erosion, and the parameters that would affect the erosion. Furthermore, the application of masks in controlling the eroded footprint and the areas in which AJM could improve to which the current proposed method would contribute. The previous research which has established the foundation of the current method, including the models which predict the time-evolution of surfaces machined using AJM and other inverse methods, will be presented in Section 2.1.

\subsubsection{Application of Microchannels and Features Created with AJM}

Microfluidic circuits have been a subject of interest in filtering, manipulation, and sorting beads and cells, and have proven their application in modeling the behaviour of microorganisms and organs [1]. This has facilitated accurate predictions of medicinal side effects and the response of organs in medical fields such as cordial arteries, thanks to its high precision and reliability [7]; other areas of application include fabricating collagen-alginate composites [8] and flow cytometry. 
The aforementioned microfluidic devices, also known as "lab-on-a-chip", usually comprised of pumps, dispensers, and separators [7]. A feature of these devices is their ability to confine the flow and limit the floating particles' motion to the center of the channel, which help prevent clogging and deterioration of what is carried by the flow [1], which motivated application of methods such as flow cytometry used in studying AIDS and leukemia [9] [10] [11]. Flow cytometry has a lower cost and required sample size compared to alternative methods for flow confinement [12].

\subsubsection{Methods of Machining Microchannels}

Conventional microfabrication methods include wet etching, soft photolithography, micromilling, EDM, and LASER machining. These each have specific drawbacks; be it wet etching's chemical contamination of the target's surface and the hazards involved with the chemical being used in the process [13]; the micro-milling's time, the rigidness of the material used to create its bit and the required precision of tooling bits make it disadvantageous, and, it could cause defects in micro-scale in the substrate [14] [15]; EDM's electrode wear and cracking of the workpiece [16] [3]; or, laser machining's large heat affected zones and substrate damage [17].

In most of the channels created using the above methods, fluid and particles flow in a single direction. However, their application in health care could be extended by featuring variations in their microchannels that could direct the flow in three-dimensions [18]; e.g. soft photolithography [1] and curing polydimethylsiloxane (PDMS) in microchannel molds [19] have been used to create 3D features which focus the microflow. Yet, these methods require precise alignment of the mask and the location of the intended feature on the micro-scale. Moreover, the produced feature may have a varying roughness that would affect the repeatability of the manufacturing process.

\subsubsection{Benefits of using AJM and the Parameters that Influence it}

As an alternative, abrasive jet micro-machining could be used to machine MEMS, microfluidic and optoelectronic devices, which, not only is not hindered by the previous methods' shortcomings but also can machine anisotropically; this is not possible using isotropic etching techniques such as, for example, wet etching [20] [21]. AJM uses pressurized fluid to carry microparticles which are accelerated and blasted on the target material to be machined, resulting in its erosion over time [20]. Some of the parameters influencing the shape of the final feature being machined using AJM include the target is brittleness or ductility, its fracture toughness, hardness and elasticity, the air pressure and dimensions of the nozzle spraying the combination of the fluid and the particles, the location of the nozzle relative to the target's surface, the roundness and size of the abrasive particles as well as the direction at which these particles, impact the target's surface [22] [23] [24].

\subsubsection{Mechanisms of Erosion}

To understand erosion (the mechanism through which AJM removes material) it is essential to study the behavior of target material under impact by abrasive particles. The brittle 
target deforms elastically upon impact until the fracture occurs and cracks propagate radially and laterally [25], causing chipping to occur, and consequently, erosion [26] [27] [28]. On the other hand, impact with a ductile target can result in embedment of particles before passing the threshold of static friction [29]. However, beyond this point, ductile erosion takes place, as the result of the combined effect of several deformation and material removal mechanisms such as pitting, extrusion and cutting [30] which result in crater lip formation and ploughing the material away with consequent impact of particles [31] [32] [33]. Erosion in brittle material is maximum in the direction normal to the surface [34] while that in ductile material is highest in oblique directions, usually between $15^{\circ}$ to $30^{\circ}$ to the surface [35]. A partial transition takes place from ductile to brittle erosion as the abrasive particles decrease in size or the temperature of the carrier fluid drops [36] [37].

\subsubsection{Surface Quality of Channels Machined in AJM}

The quality of the machined surfaces using AJM is a property of note since increased roughness could adversely affect the adhesion of glass coatings applied to the machined surface [38], and the pressure drop within micro-channels [39]. It was observed that roughness, which was calculated based on the lateral crack length in ceramics [26], was exponentially related to the particles' impact energy [34]. In addition, this model was modified to account for shape size, velocity, dose, the angle of incidence and possibility of fragmentation of the erodent particles to calculate roughness [40] [41] and waviness [42]. Moreover, post-blasting the specimen with smaller particles[43] after being machined also helped reduce the roughness, but this procedure was deemed inefficient for machining shallower channels [44]. Among other methods introduced to reduce the roughness of the AJM machined channels were HF etching (which was less effective) and annealing [43].

\subsubsection{Application of Masks in AJM}

The AJM process is not without its limitations and flaws. The particles sprayed from the nozzle diverge more with increasing distance from the nozzle which reduces the resolution of the features that can be machined [45] [46]. To reduce the size of the eroded footprint and create a more uniform distribution of the particles' velocity, researchers have masks applied directly to the surface or suspended above it [47] [48]. However, this enhancement requires precautions since the edge of the mask can deflect the incoming particles and reducing the erosion at that point, undercutting (in case of metal masks) and possible frosting (in case of using suspended shadow masks) [48] [49]. Some variants of the masks that adhere to the surface were the sacrificial metal mask [50] and the photoresist mask. The latter was cured under ultraviolet light at predetermined locations and adhered to the surface, which was eroded faster at the locations exposed to the UV light which has been used to machine features as small as $50 \mu \mathrm{m}$ [51] [52]. The photoresist masks have also been used as suspended shadow masks in combination with rotating apparatuses to machine W-shaped features in the glass [53]. 


\subsection{Summary}

The previous section illustrated the significance of AJM as a micro-machining method through its application and advantage compared to its alternatives. Furthermore, the erosion of both ductile and brittle material, and the parameters that influence it, were described briefly as well as the mechanisms which lead to each category of mechanical response. Moreover, the effect of surface quality on the micro-machined channels and methods to improve it were recounted. Finally, the integration of sacrificial and shadow mask was accounted for as improvements in the resolution of eroded features and controlling the distribution of erodent. However, no predetermined feature created with AJM has previously reached a relatively high aspect ratio (previously, the topographies were controlled with aspect ratios up to 0.05) (A.R.). In this thesis, an inverse method was developed and verified to address this. The method bore similarity to Newton-Raphson used to find the roots to a function which earned motivated its reference to Newton's efforts in mathematics.

\subsection{Objectives}

The objectives of this thesis were to:

(i) Develop a method to predict the distribution of erosive footprint required to machine a predetermined cross-section in a microchannel.

(ii) Implement the overlapping adjacent pass method to machine channels of high A.R. (see section 2.4) in both brittle and ductile targets. 


\section{Theory}

The following chapter is based on the following journal article (submitted):

Aria Ghazavi, Marcello Papini, (2018), An Inverse Method for the Abrasive jet micro-machining of High Aspect Ratio Channels of Desired Topography - Part I, Theory

\subsection{Introduction}

AJM is a non-conventional machining technique with applications in micro-technology, in particular in the fabrication of microfluidic lab-on-a-chip devices [54]. It uses compressed air to accelerate micro-scale particles through a nozzle and onto a target material, where the material is removed through solid particle erosion mechanisms. For micro-machining, patterned erosion resistant masks clamped or adhered to the target surface are often used to limit the size of the erosive footprint and thus machine small features[55]. Alternatively, the footprint of the jet can be limited by positioning the nozzle close to the target's surface; e.g., an AJM rectangular nozzle of $200 \mu \mathrm{m}$ width at a $130 \mu \mathrm{m}$ stand-off has been used to produce a $420 \mu \mathrm{m}$ wide foot-print [56] [57].

The high-speed collisions of the particles during AJM remove target material through wellknown solid particle erosion mechanisms [58]. Although a variety of material removal mechanisms have been proposed and modeled, it is generally accepted that erosion occurs either through brittle erosive processes (deformation, cracking, chip removal) or ductile ones (ploughing, cutting, etc.) [59] [60] [61] [62]. In general, brittle erosive systems have maximal erosion when the particles arrive perpendicular to the surface, while ductile systems erode more rapidly at oblique incidence [63]. Modeling of AJM has thus focused on the prediction of the time-dependent evolving eroded profile which occurs because the local angle of incidence of particle impacts changes as the eroded features develop sloped sidewalls [64].

ten Thije Boonkkamp and Jansen were the first to consider a surface evolution model, assuming that only the component of the particle velocity normal to the target's instantaneous surface profile contributes to erosion in a brittle material [64]. They derived a non-linear partial differential equation (PDE) that can be used to predict the evolution of an eroded profile assuming the abrasive particle flux and velocity, as well as the dependence of the target erosion on these factors, are known. Getu et al. later extended this model for ductile erosive systems, where both tangential and normal components of incident velocity contributed to erosion and used it to predict the shapes of channels and holes machined using AJM in polymers [65]. Ally et al. used a similar model to predict the surface evolution of channels and holes in metals [66]. Later refinements include the use of an 'erosive efficacy' by Ghobeity et al. to describe the potential of the abrasive jet to erode the target surface [57] [67], and the use of a viscous smoothing term [68] that avoided singularities near the sharp cusps that were predicted to develop during surface evolution of masked targets. Since the erosive efficacy could be easily determined from a shallow single pass profile of the eroded surface [56], it greatly simplified the modeling process. More advanced numerical and simulation techniques such as the level set [69] and cellular automata based methods [70] have also been used to solve the surface evolution equation for both brittle and ductile erosive systems, including such effects as the particle to mask and particle to surface ricochets. 
Very few attempts have been made to manipulate the process parameters in order to obtain a particular desired feature topography. Most of these have used shallow adjacent raster passes to sculpt relatively shallow features of the desired shape, thus avoiding complications associated with changes in the local angle of attack brought about by significantly sloped sidewalls. For example, Ghobeity et al. used shallow adjacent AJM passes and oscillating nozzles to machine large flat areas in the glass [71]. Sookhak Lari et al. used AJM with a patterned rotating shadow mask in order to machine shallow channels of prescribed cross-sectional shapes (W-shaped and trapezoidal) in the glass [72]. However, their method assumed that significant sidewall slopes did not develop so that the machined surface evolution progressed linearly. This limited the maximum aspect ratio (A.R., the ratio of feature depth to width) of their desired shapes, i.e. $1 \mathrm{~mm}$ wide by $100 \mu \mathrm{m}$ deep (A.R.=0.1) W-shapes, and $1.8 \mathrm{~mm}$ wide by $60 \mu \mathrm{m}$ deep trapezoidal shapes (A.R.=0.03). Sookhak Lari and Papini also developed a technique that allowed various desired channel centerline textures to be created by varying the scan speed along the length of the channel [57]. The methodology, however, again assumed negligible sidewall slope so that the depths of the textures were limited to the order of 100-200 $\mu \mathrm{m}$ over wavelengths of 2-6 mm (A.R.s between 0.03 and 0.05 ). Some related work has been performed in abrasive water (AWJM) and slurry jet (ASJM) machining, which uses water instead of air as the fluid to accelerate the abrasive. For example, Billingham et al. studied the effect of overlapping AWJM sources, creating trenches with uniform cross-sections in Ti6Al4V that remained at a constant depth and ones that showed rise and falls in the depth up to 0.25 aspect ratio. Meanwhile, their model could predict the evolution under overlapping AWJM sources at aspect ratios between 0.1 and 0.23 [73]. Axinte et al. used an inverse technique to successfully machine a shallow 3D pattern of a British Penny and the Mona Lisa on Ti6Al4V [6]. Finally, Tamannaee et al. used adjacent ASJM passes to machine sharp edges on machined talc olefins to various desired radii [74]. None of these studies considered the effect of the change in erosion rate that occurs on the ever-steepening sidewall slopes as the surface erodes.

In summary, while the prediction of the eroded topography of features resulting naturally from masked and unmasked AJM is well-established, only a few attempts have been made to sculpt desired topographies, and those have focused only on the shallow, low A.R. case.

However, in some applications, higher A.R. channels of prescribed shape are required. For example, perfusion channels with uniform cross-sections and A.R.=1 were previously proposed by Rainer et al.'s method to deplete red blood cells at high flow rates [75]. Another example of the significance of controlling the cross-section of a micro-channels with ARs as high as 0.65 was demonstrated by $\mathrm{Wu}$ and Cheng, who studied the pumping power per temperature difference of trapezoidal silicon ducts, and concluded that steeper sidewalls would improve the heat transfer rate [2].

This thesis presents a simple inverse methodology to determine the erosive efficacy and machining path required to produce channels with prescribed cross-sectional shapes at much higher ARs (up to 0.5). In contrast to previous techniques, the present method considers the effect of the changing erosion rate brought about by the evolution of the sidewall slope by using an iterative inverse solution to the partial differential equation of surface evolution. The method thus makes possible the design of micro-devices with high aspect ratio features with a wide variety of topographies. 


\section{2. $\quad$ Modeling}

The objective was to determine the distribution of erosive efficacy[76] (section 2.3) required to machine a desired high aspect ratio channel cross-sectional profile using a predetermined number of adjacent, partially overlapping, nozzle passes at different scan (traverse) speeds. As mentioned in section 2.1, in previous works that considered only very shallow desired topographies, the dependence of local erosion rate on eroded feature sidewall slope could be neglected so that the inverse problem could be represented as an integral equation to be solved in closed-form [20]. This work will concentrate on the more difficult inverse problem associated with machining much deeper features that develop steep sidewalls such that the local particle angle of attack varies across the eroded feature width. This leads to a nonlinear relationship between profile shape (depth $\xi$, at any lateral point, $x$ ) and time which precludes the use of closed-form solutions. Thus, an iterative technique was developed to solve this much more complex inverse problem.

\subsection{Surface Evolution Modeling}

Surface evolution models can be used to predict the instantaneous cross-sectional profile of a channel machined using AJM. In the present work, the forms of the surface evolution models for brittle and ductile erosive systems as presented by Ghobeity et al. were used. For brittle materials, the model reduces to [20]

$\frac{\partial \xi(x, t)}{\partial t}-E(x)\left(1+\left(\frac{\partial \xi(x, t)}{\partial x}\right)^{2}\right)^{-\frac{k}{2}}\left(1-\epsilon \cdot \frac{\frac{\partial^{2} \xi(x, t)}{\partial x^{2}}}{\left(1+\left(\frac{\partial \xi(x, t)}{\partial x}\right)^{2}\right)^{\frac{3}{2}}}\right)=0$

where $\xi(x, t)$ is the instantaneous depth of the eroded surface, $t$ the time the nozzle spends dwelling over the lateral location $x, k$ is an experimentally-determined velocity exponent measured to be $\sim 1.43$ for borosilicate glass[77], and $\in$ is a viscous smoothing factor incorporated to avoid numerical instabilities at unrealistically sharp cusps that the model would predict.

The erosive efficacy function $E(x)$ characterizes the distribution of the potential to erode the surface [5], and as defined in Equation 2-1, has dimensions of length per time. It depends on the distribution of particle size, shape, velocity, and flux, as well as the target erosive properties.

As a special case, if the surface remains relatively flat (shallow erosion scar), or if the profile is symmetric at its axis so that $\partial \xi / \partial x=0$, then

$$
\frac{\partial \xi(x, t)}{\partial t}=E(x)
$$


Ghobeity et al. [20] also demonstrated that, if the number of times a single nozzle passed over the location at which $x=0$ was denoted by $n$, it would establish the following relation, $t^{*}=n . \zeta^{*}$, between normalized predicted depth $\zeta^{*}$, which was $\zeta_{1}$ (the maximum depth of the nozzle's first pass) divided by the nozzle's off-set height from the uneroded surface of the target $(h)$ and the normalized time $t^{*}$ (ratio of time over the characteristic time $T$ ).

Hence, by applying the chain rule differentiation with respect to $n$, expanding the normalized terms involved, and multiplying both sides of the equation by $\left(\zeta_{1} . T\right) / n$, one would conclude:

$$
\frac{\partial \xi(x, n)}{\partial n}-\frac{\partial t}{\partial n} E(x)\left(1+\left(\frac{\partial \xi(x, n)}{\partial x}\right)^{2}\right)^{-\frac{k}{2}}\left(1-\epsilon \cdot \frac{\frac{\partial^{2} \xi(x, n)}{\partial x^{2}}}{\left(1+\left(\frac{\partial \xi(x, n)}{\partial x}\right)^{2}\right)^{\frac{3}{2}}}\right)=0
$$

In the equation above, the term $\partial t / \partial n$ represents the time required to complete one pass by the nozzle and its product with the erosive efficacy $E(x)$ would hitherto be referred to as the 'scaled erosive efficacy' $E_{s}(x)$ This parameter could be extracted from a shallow scar with a non-contact profilometer (NANOVEA ST400 Micro Photonics Inc., Irvine, CA, USA). Moreover, for smaller slopes, the depth of the eroded surface is linearly proportional to the number of passes. Therefore, the scaled erosive efficacy can be extracted from the eroded profile of a shallow channel as described by Ghobeity et al. [20].

For ductile erosive systems, the surface evolution equation, as described by Getu et al. [65] [47] using the effect of the change in angle of attack as noted by Oka et al. [35], is

$$
\begin{aligned}
& \left(\frac{\partial \xi(x, n)}{\partial n}-\frac{\partial t}{\partial n} E(x) \sqrt{1+\left(\frac{\partial \xi(x, n)}{\partial x}\right)^{2}} g(\alpha)\left(1-\epsilon \cdot \frac{\frac{\partial^{2} \xi(x, n)}{\partial x^{2}}}{\left(1+\left(\frac{\partial \xi(x, n)}{\partial x}\right)^{2}\right)^{\frac{3}{2}}}\right)=0\right. \\
& g(\alpha)=\sin ^{k_{1}}(\mu)\left(1+H_{v}(1-\sin (\mu))\right)^{k_{2}} \\
& \mu=\frac{\pi}{2}-\arccos \left(\frac{1}{\sqrt{1+\left(\frac{\partial \xi(x, n)}{\partial x}\right)^{2}}}\right)
\end{aligned}
$$

where, the experimentally determined constants $k_{1}, k_{2}$ and $H_{v}$ were previously reported as 1.27 , 0.23 and 15.5, respectively, for PMMA (poly-methyl-methacrylate). For a shallow erosion scar, 
the Equation 2-4 reduces to describe a linear propagation of the eroded front, similar to Equation 2-3 so that the scaled erosive efficacy $\left(E_{s}(x)=\partial t / \partial n . E(x)\right)$ can again be extracted from a shallow profile. Similarly, the rate at which the surface erodes with respect to the number of passes could be related to the scaled erosive efficacy as seen in equation set 2-4 (see section 2.4 which demonstrates the extraction of $E_{s}(x)$ could be measured by the same procedure for PMMA).

Both of the PDEs in Equations 2-3 and 2-4 are non-linear. Hence, the inverse problem of deriving an analytical expression for the total erosive efficacy required to arrive at a desired evolved surface is intractable. This is in contrast to earlier work [5] [53] where the desired feature was shallow and the problem was thus linear. Consequently, the iterative algorithm of Figure 2.3.1was used.

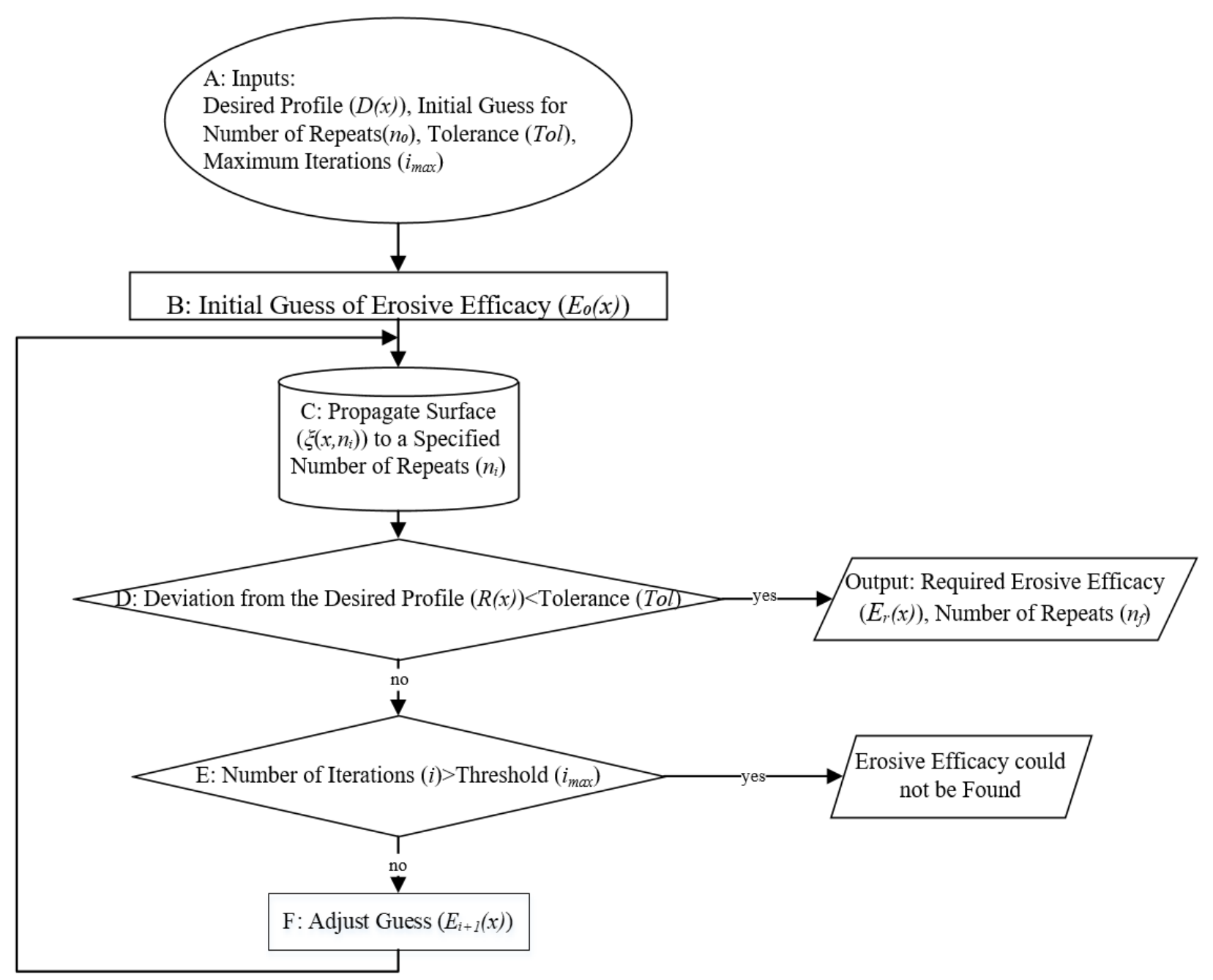

Figure 2.3.1: Summary of the inverse method to determine the scaled erosive efficacy required to machine the desired surface shape 


\subsection{Deviation from Linear Surface Evolution}

As discussed in Section 2.1, inverse models already exist to machine shallow channels of prescribed topography. Yet, these models could only be applied to "shallow profile" channels. It remained to be determined to what range of A.R. the previous models would apply, neglecting the dependence of erosion rate on the local angle strikes on the sloped feature sidewalls, and which range would require the new inverse model.

Since the presently proposed methodology increases the computational cost compared to such simplified models, assuming the surface evolves linearly with time, it was of interest to determine how rapidly the surface evolution deviates from the linear assumption. Therefore, a comparison was made between the more accurate predictions (resulted from solving Equation 2-3 or 2-4 as the channel becomes deeper) and the linearly scaled cross-sectional profile of straight channels machined (in the glass and PMMA by a single nozzle pass) with respect to its A.R.. Figure 2.4.1 shows that even for an AR of 0.06, an average 10\% difference in predicted profiles already exists. The difference very rapidly increases with $\mathrm{AR}$, and was more accentuated for the ductile PMMA. Therefore, it is clear that the more complex approach is necessary.

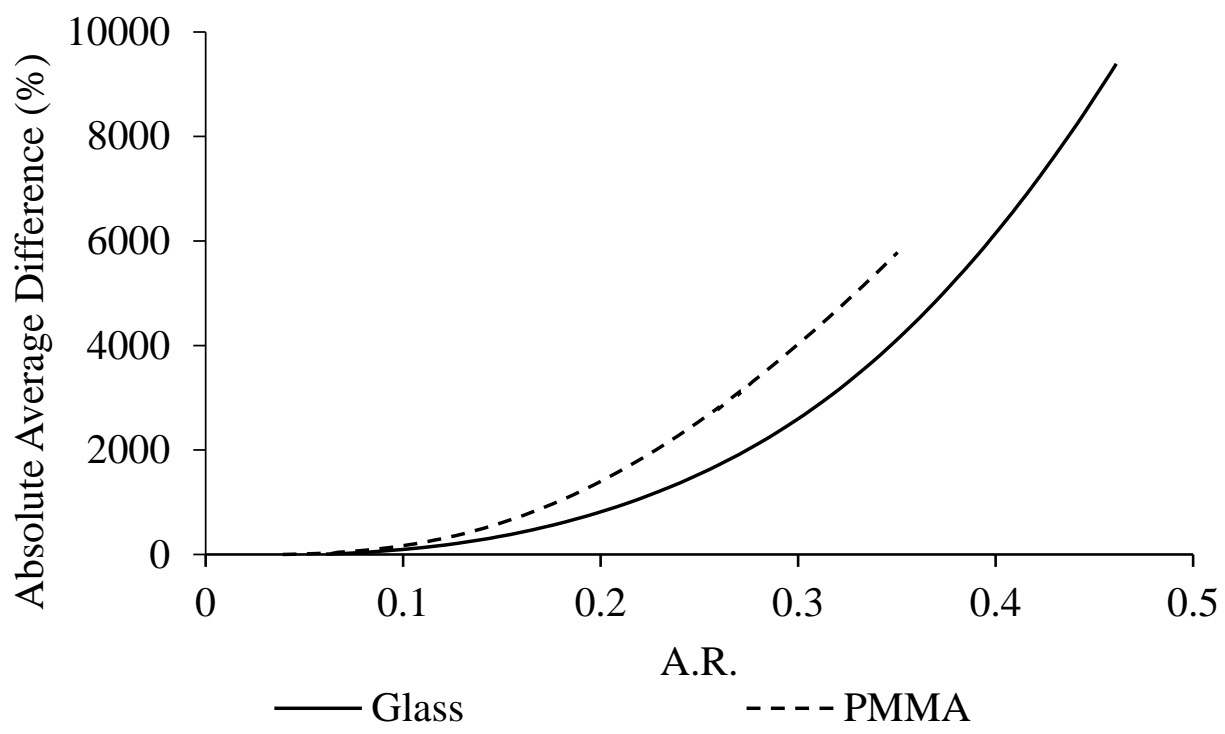

Figure 2.4.1: Average absolute percentage difference between predicted profiles of a single-source straight channel evolving to a given aspect ratio when assuming a linearly scaled surface evolution, and its evolution according to equations 2-3 and 2-4.

\subsection{Inverse Modeling}

An iterative inverse method was used to predict the required scaled erosive efficacy $E_{r}(x)$, and the number of repetitions, $n_{f}$, of a series of overlapping adjacent passes of the nozzle (Figure 2.5.1) required to machine a given desired profile $D(x)$. Every iteration, current 'guesses' of the required $E_{r}(x)$ and $n$ were used in the surface evolution Equations 2-3 and 2-4, to predict the profile. The algorithm improved the previous guesses for $E_{i}(x)$ and $n$ every iteration until the final profile matched the desired one to within a tolerance. 


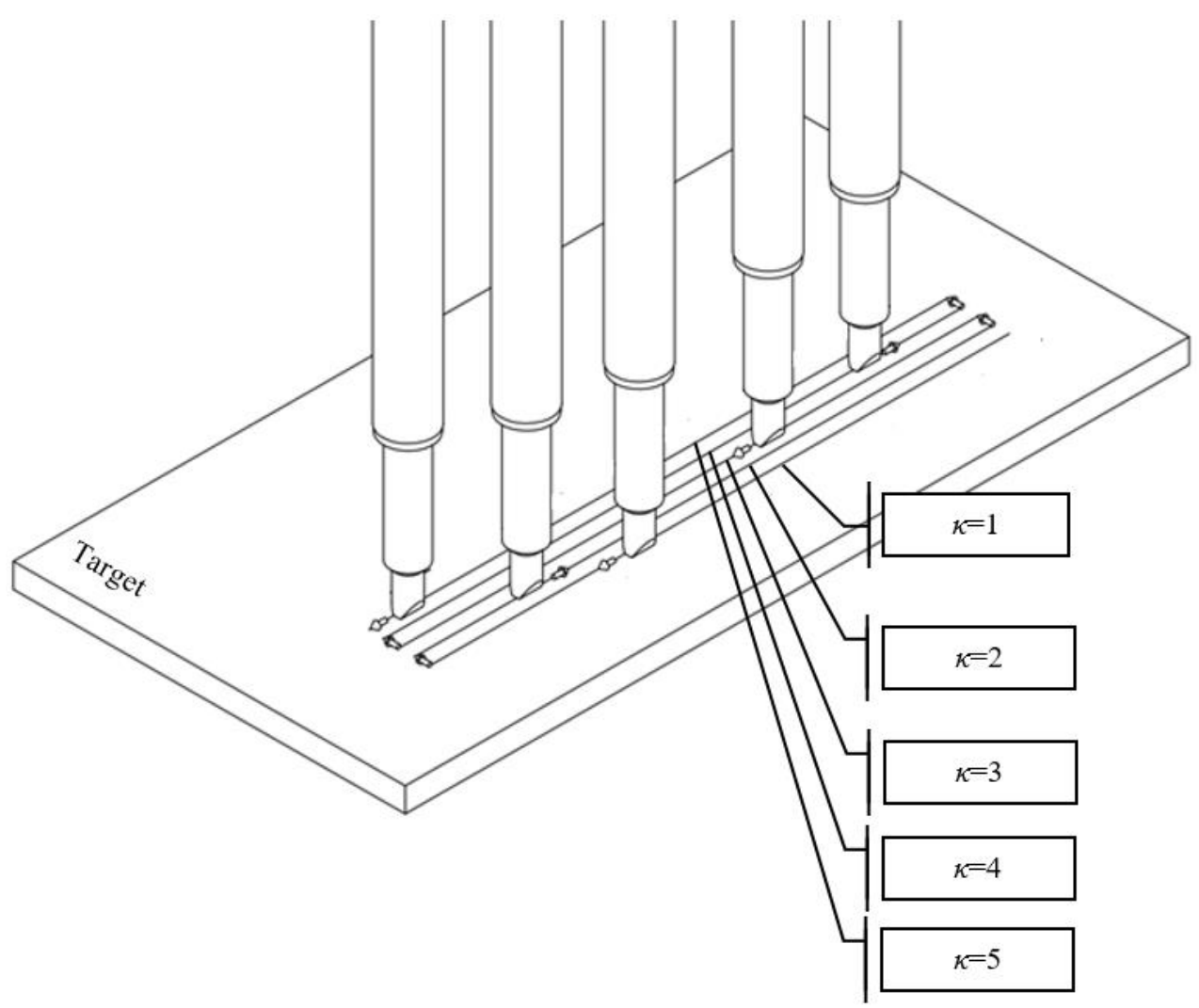

a)

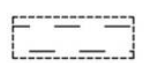

Pass 1's Erosive Efficacy

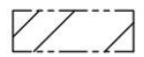

Pass 2's Erosive Efficacy
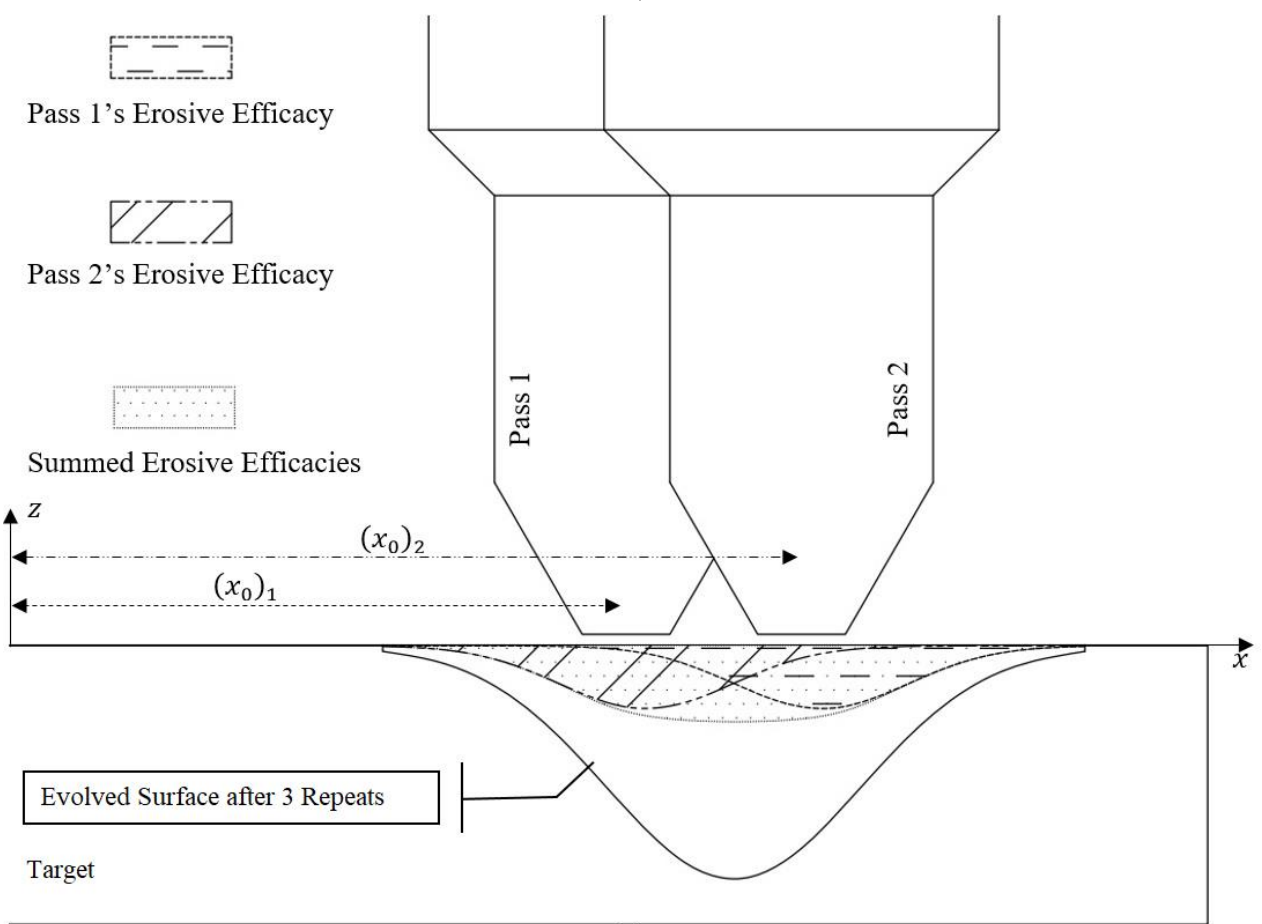

b)

Figure 2.5.1:(a) Schematic drawing of multiple adjacent passes of the source (nozzle) needed to produce a channel profile of prescribed shape and (b) the scaled erosive efficacies of two adjacent passes 1 and 2 which superimpose to yield the deeper profile. The evolved surface resulting from multiple repetitions of the adjacent passes is also shown. 


\subsubsection{Inputs (Block A)}

The model takes as input the desired cross-sectional profile $D(x)$ and the maximum tolerance $T o l$ for depth deviation from $D(x)$. To prevent perpetual iterations for cases in which the algorithm cannot resolve $E_{r}(x)$, (the required erosive efficacy to machine $D(x)$ ), the user also specifies a maximum number of iterations, $i_{\max }$.

\subsubsection{Initial Guess (Block B)}

The initial guess $E_{0}(x)$ of the required scaled erosive efficacy assumed that the desired profile depth $D(x)$ was a linear function of the number of adjacent pass repeats, $n_{0}$, required to produce it, i.e., the guess neglected the development of the sidewall slope on the machined feature so that $D(x)=E_{o}(x) n_{0}$. It was thus convenient to define the ratio, $d_{o}$ between the maximum depths of the nozzle scaled erosive efficacy and that of the desired profile as

$$
d_{0}=\frac{\min \left(E_{j}(x)\right)}{\min (D(x))}=\frac{1}{n_{0}}
$$

$E_{j}$ is the jet's scaled erosive efficacy (AKA the source's scaled erosive efficacy) discussed in Section 2.6.1). no was thus an estimate of the number of repeats required to machine the profile to the desired depth. The initial guess was then determined as

$$
E_{0}=d_{0} D(x)
$$

This initial guess for the scaled erosive efficacy was used in the surface evolution equation to predict the evolved surface in the first iteration $\xi_{I}\left(x, n_{0}\right)$.

\subsubsection{Propagate Surface Based on Current Guess (Block C)}

For each iteration $n$, the nonlinear partial differential Equations 2-3 and 2-4 were solved numerically using the current guesses for the scaled erosive efficacy and number of repeats, $E_{i}(x)$ and $n_{i}$ (Section 2.5.5 to yield the evolved profile $\xi_{i}\left(x, n_{i}\right)$. The differential equations were solved using the method of lines implementation in Mathcad®) (Version 15.0, PTC Inc., Needham, MA, US). A total of 200 space $(x)$ and 100 repeat $(n)$ steps were used within the width of the desired profile $W$, and between 0 and $n_{i}$, respectively. As an initial condition for the $n$th iteration, the surface's location after the first pass was assumed to be described by $E_{i}(x)$ i.e., $\xi_{i}(x, 1)=E_{i}(x)$. 


\subsubsection{Halting Conditions (Blocks D and E)}

The algorithm halted if one of two following conditions occurred:

(i) The maximum difference between the current profile $\xi_{i}\left(x, n_{i}\right)$ and the desired $D(x)$ was less than or equal to the specified tolerance (typically chosen to be $10 \%$ of the maximum depth in the target), i.e.

$\max \left|\xi_{i}\left(x, n_{i}\right)-D(x)\right| \leq T o l$

In this case, $E_{i}(x)$ was returned as a suitable approximation of the final scaled erosive efficacy, $E_{r}(x)$ required to erode the surface to the desired shape $D(x)$ after $n_{i}$ repeats, after which the surface would be described by $\xi\left(x, n_{f}\right)$.

(ii) The number of iterations $i$ exceeded $i_{\text {max }}$ (typically 100), in which case it was concluded that the required erosive efficacy could not be determined with the given inputs.

\subsubsection{Adjustment of Scaled Erosive Efficacy Each Iteration (Block F)}

As explained in Section 2.5.3, Equations 2-3 and 2-4 were used to predict the evolved surface after each iteration, $\xi_{i}\left(x, n_{i}\right)$. If neither of the stopping conditions of Section 2.5.4 were met, the following adjustments were made to the scaled erosive efficacy and the required number of repeats,

$$
\left\{\begin{array}{c}
E_{i}(x)=E_{i-1}(x)+d_{i-1}\left(D(x)-\xi_{i-1}\left(x, n_{i-1}\right)\right), i \geq 1 \\
d_{i}=\frac{E_{i-1}(0)}{D(0)}=\delta / n_{i}, i \geq 1 \mid D(-x)=D(x) \\
d_{i}=\frac{E_{i-1}\left(x_{\text {min }}\right)}{D\left(x_{\text {min }}\right)}=\delta / n_{i}, i \geq 1 \mid D\left(x_{\text {min }}\right)=\min (D(x))
\end{array}\right.
$$

where the subscripts $i$ and $i-1$ indicate the current and previous iterations, respectively. The second equation in the set 2-8 was used for symmetric features (when the derivative at the center is zero), and the third equation in the equation set 2-8 was used for asymmetric features.

To avoid excessively large corrections to the scaled erosive efficacy that caused stability problems in the solution, the correction was scaled by a constant $\delta=0.01$, a value that was determined through trial and error. In this manner, the $x$ dependency of the correction was maintained, but the magnitude of the correction applied in each iteration was limited.

This adjustment was reminiscent of the Newton-Raphson's method of finding roots with the surface evolution equation's deviation from the desired being the function the roots of which $\left(E_{r}(x) \mathrm{s}\right)$ were to be determined by the algorithm. This inspired the association of the inverse algorithm with Newton's mathematical endeavors. 


\subsection{Generation of Required Erosive Efficacy Using Adjacent Passes}

The algorithm of Section 2.5 predicted the required erosive efficacy $E_{r}(x)$, and $n f$, the predicted number of repeats required to machine the desired deep profile. To actually machine the profile, however, requires a machining procedure that can yield the $\operatorname{Pr}_{r}(x)$. The required $E_{r}(x)$ was approximated as $S(x)$ by superimposing multiple adjacent, partially overlapping passes of a source, as shown in Figure 2.5.1.

\subsubsection{Source Scaled Erosive Efficacy, $E_{j}(x)$}

The source scaled erosive efficacy $E_{j}(x)$ delivered in a single straight pass of the nozzle on borosilicate glass are shown in Figure 2.6.1 a for various scan speeds. They were simply extracted as measured profiles of shallow channels. Sookhak Lari and Papini found that, for nozzles typical of AJM applications eroding glass targets, $E_{j}(x)$ can be fit to the following distribution with a magnitude proportional to a 'source strength' $K_{s}$, defined as the product of the centerline depth and the nozzle scan speed $v_{s}$ so that, if scaled by $\partial t / \partial n[5]$

$E_{j}(x)=\frac{\partial t}{\partial n} \frac{K_{s}}{v_{s}} e^{-x^{2} /\left(h / \beta_{w}\right)^{2}}$

where $h$ is the nozzle to target standoff distance, and $\beta_{w}$ is a fitting parameter. Figure 2.6.1(c) shows the footprint etched on a glass target based on the average depths in Figure 2.6.1(a), together with the least squares best fit to Equation 2-9. For a ductile sample, however, the shallow profile is more U-shaped [65], and it was therefore fit to an eighth order Fourier series:

$E_{s}=\left(\frac{\partial t}{\partial n} \frac{K_{s}}{v_{s}}\right) \frac{a_{0}+\sum_{\gamma=1}^{8}\left(a_{\gamma} \cos \left(\gamma \omega_{0} x\right)+b_{\gamma} \sin \left(\gamma \omega_{0} x\right)\right)}{\max \left(a_{0}+\sum_{\gamma=1}^{8}\left(a_{\gamma} \cos \left(\gamma \omega_{0} x\right)+b_{\gamma} \sin \left(\gamma \omega_{0} x\right)\right)\right)} \cdot \Pi\left(\frac{x}{W}\right)$

where $a_{0}, a_{\gamma}$ and $b_{\gamma}$ (for $\gamma=1,2, \ldots, 8$ ) are the Fourier Coefficients, and $\omega_{0}$ the Fourier frequency and $\Pi(x / W)$ the rectangle function limiting the span to $W$ the range between the two edges of the footprint (as defined previously by Nouhi et al. [48]), or its width.

Figure 2.6.1(b) and (c) show the $E_{j}(x)$ and $E_{j} v_{s}(\mathrm{x})\left(\mu \mathrm{m}^{2} / \mathrm{s}\right)$, respectively, for the PMMA targets. 


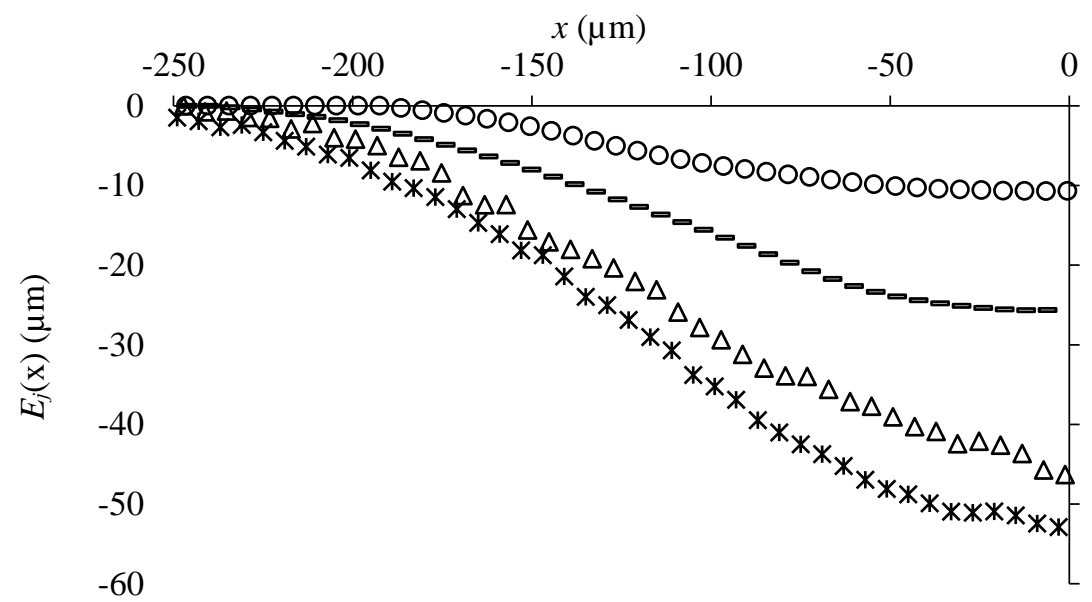

$$
O v_{s}=4 \mathrm{~mm} / \mathrm{s} \quad-v_{s}=2 \mathrm{~mm} / \mathrm{s} \quad \Delta v_{s}=1.25 \mathrm{~mm} / \mathrm{s} \quad * v_{s}=1 \mathrm{~mm} / \mathrm{s}
$$

a)

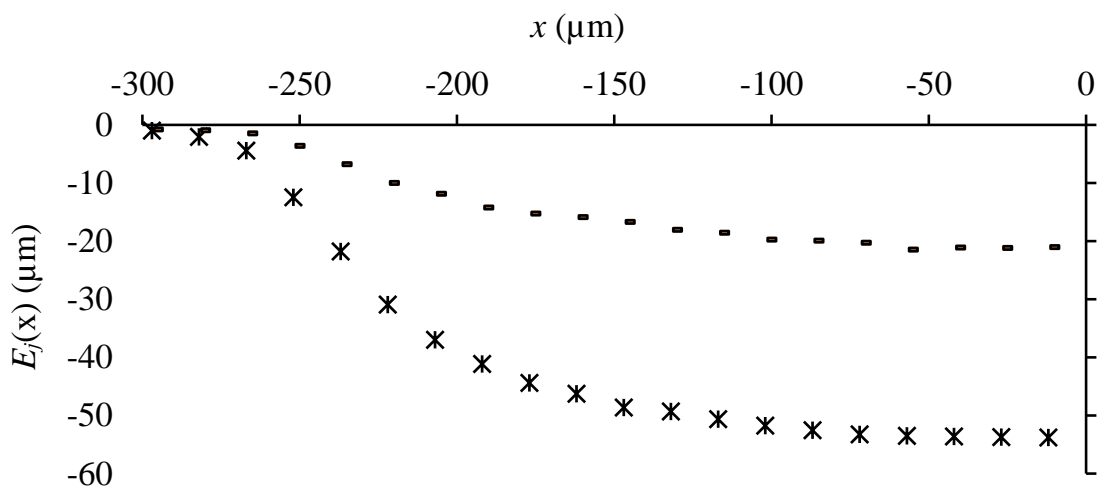

$* v_{s}=1 \mathrm{~mm} / \mathrm{s}$

- $v_{s}=2.5 \mathrm{~mm} / \mathrm{s}$

b)

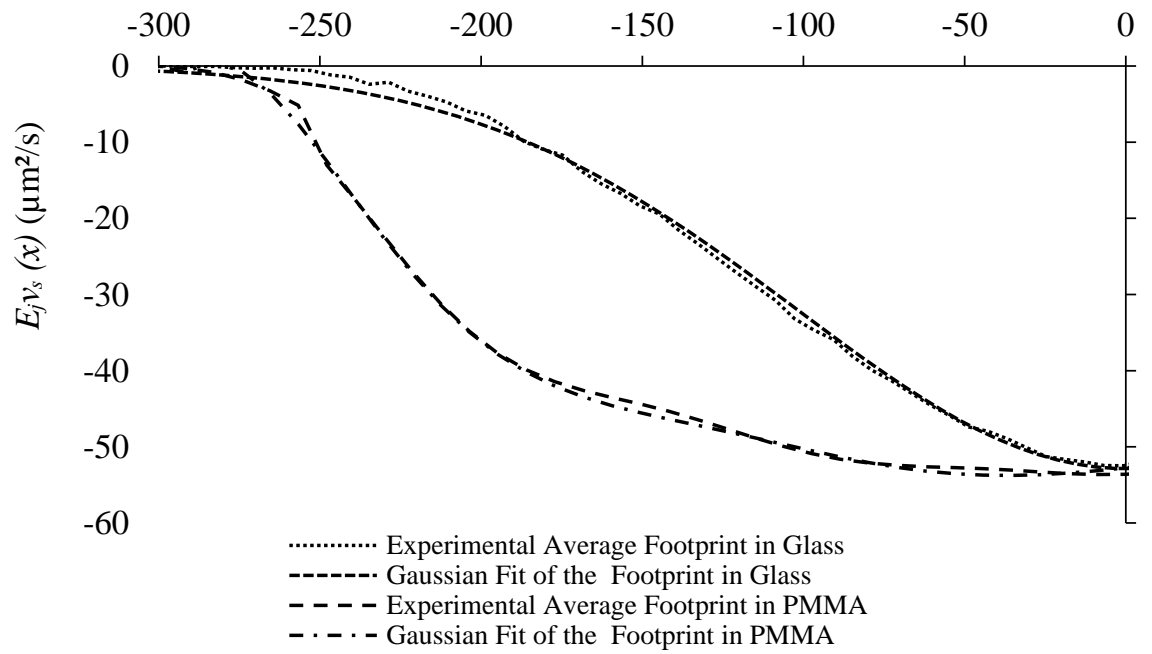

c)

Figure 2.6.1: (a) Source's scaled erosive efficacy and average source strengths on the glass and (b) PMMA, respectively (c) The curve fitted data of the average scaled erosive efficacy extracted at various scan speeds for both glass 


\subsubsection{Machining Procedure to Obtain $S(x)$}

To determine the scan speeds and offsets needed for the adjacent passes of the sources in order to approximate $E_{r}(x)$, the algorithm described by Sookhak Lari and Papini [5] was used. Briefly, given the lateral positions of the passes $\left(x_{0}\right)_{\kappa}$ and their scan speeds $\left(v_{s}\right)_{\kappa}$ the superposition of the source scaled erosive efficacies for the adjacent passes for glass yields

$$
S(x)=\sum_{\kappa=0}^{m}\left(E_{j}\right)_{\kappa}(x)=\sum_{\kappa=0}^{m} \frac{K_{s}}{\left(v_{s}\right)_{\kappa}} e^{-\left(x-\left(x_{0}\right)_{\kappa}\right)^{2} /\left(h / \beta_{w}\right)^{2}}
$$

Where $\left(E_{j}\right)_{\kappa}(x)$ is the scaled erosive efficacy contribution from the $\kappa$ th source, with constant scan speed $\left(v_{j}\right)_{\kappa}$, the locations of the offsets and the scan speeds of the adjacent passes were determined using an optimization routine that minimized $R(x)$, the difference between the required and approximated scaled erosive efficacies.

$$
R(x)=\left|E_{r}(x)-S(x)\right|
$$

Certain target topographies (i.e. $D(x)$ ) resulted in predicted required scaled erosive efficacies $E_{r}(x)$ which featured sharp changes in slope and or depth, especially near their periphery. To avoid singularities while solving the surface evolution equation, the edges of the target needed to be rounded to ensure a smooth transition in slope (Section 2.7).

With the offsets and scan speeds determined, the machining procedure was repeated $n_{f}$ (rounded to the closest even number) times to produce the desired deep feature.

\subsection{Measuring the Radius of Curvature and Identifying the Edges}

By mathematical definition, a point of inflection is identified as the location where the second derivative of a function would be zero or undefined. In reality, however, the location where the radius of curvature exceeded a threshold of several orders of magnitude higher than the median, the radius was considered to be undefined. To find these points, the radius of curvature was predicted along the cross-section for every repeat as seen in Figure 2.7.1. A trend was observed as one of the inflection points at a location with an exceedingly large radius of curvature approached the area under the nozzle's axis while the other remained at the edge (in half of the erosive efficacies span). These locations with larger radii of curvature indicated an immeasurably large $\partial^{2} \xi / \partial x^{2}$ due to the existence of a sharp cusp (as seen near the edge and at the center).

However, relative flatness (as seen between the edge and the minimum depth span with the increment of the number of passes) would also be identified by a large radius of curvature. This flatness was qualitatively demonstrated by the area between the edge and mid-span flattening. The two cases were distinguished by how rapidly their values exceeded the threshold. 


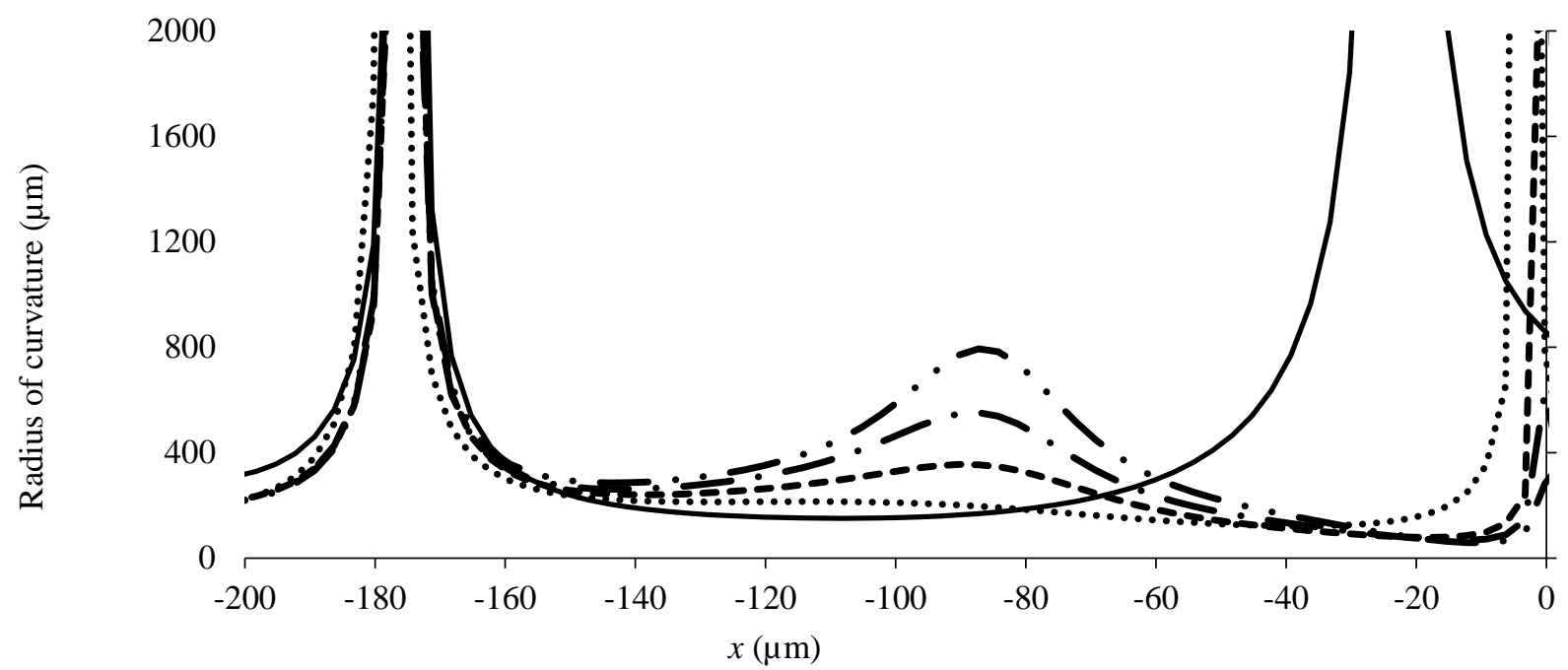

Figure 2.7.1: Radius of curvature for the single source evolution and the shift in extrema with increasing number of passes, solid line depicting the radius of curvature of the first pass at each location, the dotted line showing that of the second pass's profile, dashed line that of the third pass, dashed-dotted line that of the fourth and dashed-double-dotted that of the fifth pass's profile

To allow for a dramatic change in the profile's slope, the maximum erosion rate would be required to occur at the location with the dramatic change. Since $\partial^{2} E_{j}(x) / \partial x^{2}$ existed for each source (and hence the superposition of sources), the target's second derivative was undefined only at the sharp corners.

Furthermore, the predicted profile approached a line as the surface evolution continued with increasing number of repeats. The comparison could be seen between a line passing through two points of inflection and the evolved surfaces' predicted profile in Figure 2.7.2; the chosen points of inflection were consecutive, and the range of $x$ covered between them was the highest after the left edge and before the point with the minimum depth.

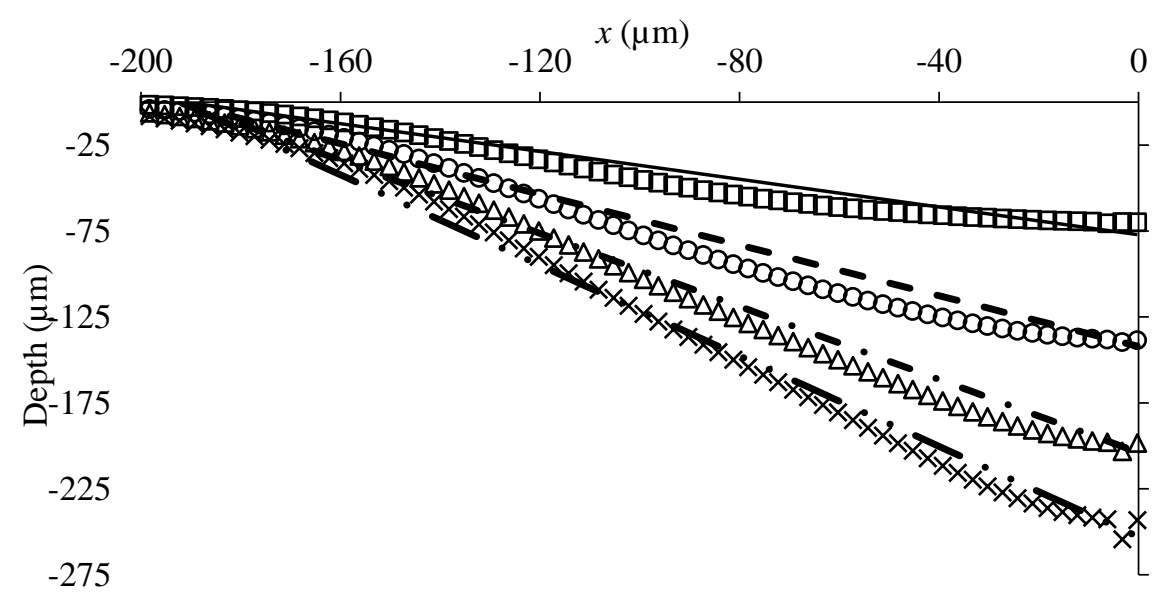

Figure 2.7.2: Half of the profile extracted from a channel eroded in the glass with nozzle scanning the specimen with $1 \mathrm{~mm} / \mathrm{s}$. Lines passing through consequent points of inflection along the profile in each repeat were drawn, proving the profile approaching a line 


\subsection{Rounding of Edges}

As discussed previously in Section 2.7, discontinuities such as sharp cusps can rapidly develop in the solution of the surface evolution equation. In reality, however, machining such cusps is impossible, and the minimum possible machinable curvature, especially at the periphery of a feature machined using the procedure of Figure 2.5.1, will depend on the shape of the source scaled erosive efficacies. To investigate this, the minimum radius of curvature was calculated by evolving (Equation 2-3) a quasi-rectangular scaled erosive efficacy depicted in Figure 2.8.1a, which was created such that the Gaussian profile in Figure 2.6.1b described its peripheral sidewalls. The mid-section was assumed constant over $\sim 600 \mu \mathrm{m}$ in order to prevent the sidewalls of the profile intersecting for profiles evolved up to a depth of $\sim 700 \mu \mathrm{m}$. The resulting minimum radius of curvature across the profile is plotted versus depth in Figure 2.8.1b, which appeared to converge to $\sim 100 \mu \mathrm{m}$. Therefore, the targets to be machined in the glass were modified to have the radius of curvature above $100 \mu \mathrm{m}$ at all locations across the profile.

For the ductile erosive PMMA, no such minimum curvature value was found. This decision was made because the flat mid-region of the profile maintained a high radius of curvature as the depth increased, while that at the edge at the periphery was small. Therefore, to ensure that the change in slope from the profile periphery to the unmachined surface was gradual, the periphery was assigned a radius of curvature $>200 \mu \mathrm{m}$. This value was obtained by trial-and-error to ensure that the algorithm remained stable.

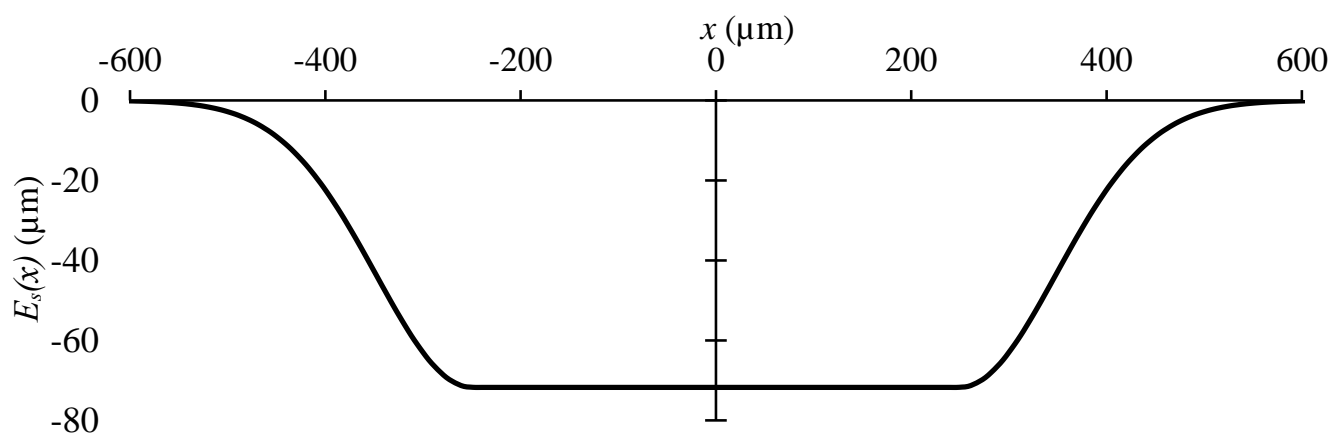

a)

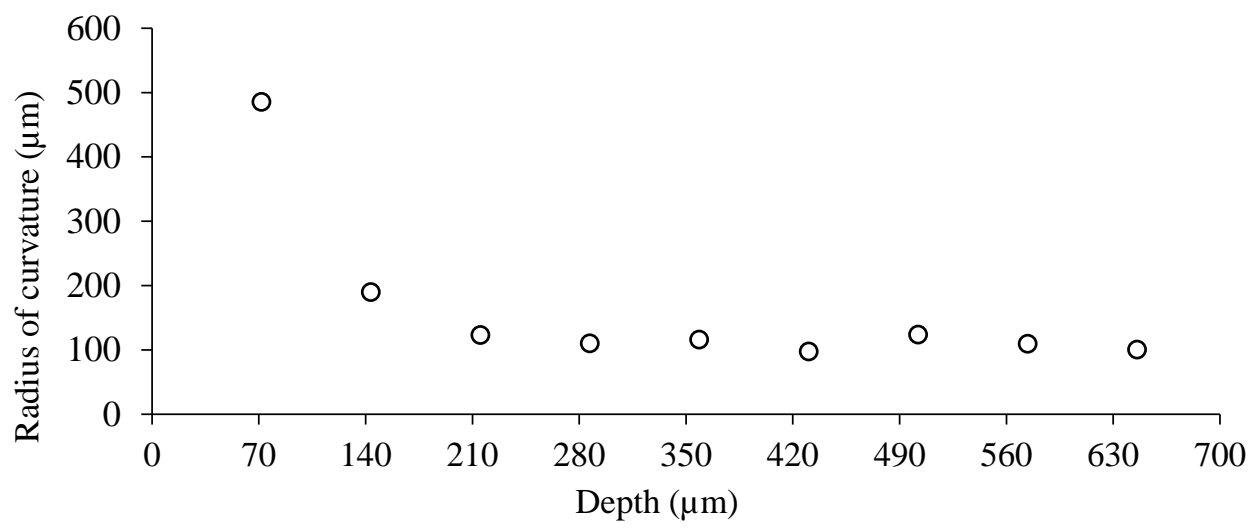

b)

Figure 2.8.1: (a) Quasi-rectangular scaled erosive efficacies, and (b) minimum radius of curvature across the profile resulting from evolving the efficacy using Equation 2-3. 


\subsection{Uniqueness and Robustness of the Inverse Solution}

The robustness of the solution predicted by the algorithm of Section 2.5 was assessed by determining whether the algorithm was capable of predicting the required scaled erosive efficacies and the appropriate number of repeats $n$, when feature topographies changed drastically during surface evolution.

\subsubsection{Predicting a Feature in $E_{r}$ Disappeared through Multiple Repeats}

For example, an initially $\mathrm{W}$-shaped profile will eventually become V-shaped, once the two sidewalls of the channel reach each other [5]. Thus, the W-shaped scaled erosive efficacies $E_{s}(x)$ arising from two slightly overlapping (300 $\mu \mathrm{m}$ apart) sources was used in the surface evolution Equation 2-3 (the product of $E_{s}(x)$ and $n_{0}$ is shown in Figure 2.9.1a), and propagated until such time that central local maximum disappeared, leaving the $\mathrm{V}$-shaped profile shown in Figure 2.9.1b. The resulting $\mathrm{V}$-shaped profile was used as $D(x)$, the desired profile in the inverse method algorithm of Section 2.5 to predict the required scaled erosive efficacies $E_{r}(x)$ and repeats $n_{f}$. This newly predicted scaled erosive efficacies scaled by its number of repeats was then compared to the original W-shaped scaled erosive efficacy multiplied by its number of repeats $\left(E_{s}(x) n_{0}\right)$, and, as Figure 2.9.1a shows, they were topographically similar, with an average absolute difference in depth of $7.7 \%$. This value was calculated by $\frac{\int\left|E_{s}(x)-E_{r}(x)\right| d x}{\int\left|E_{s}(x)\right| d x} \times 100 \%$.

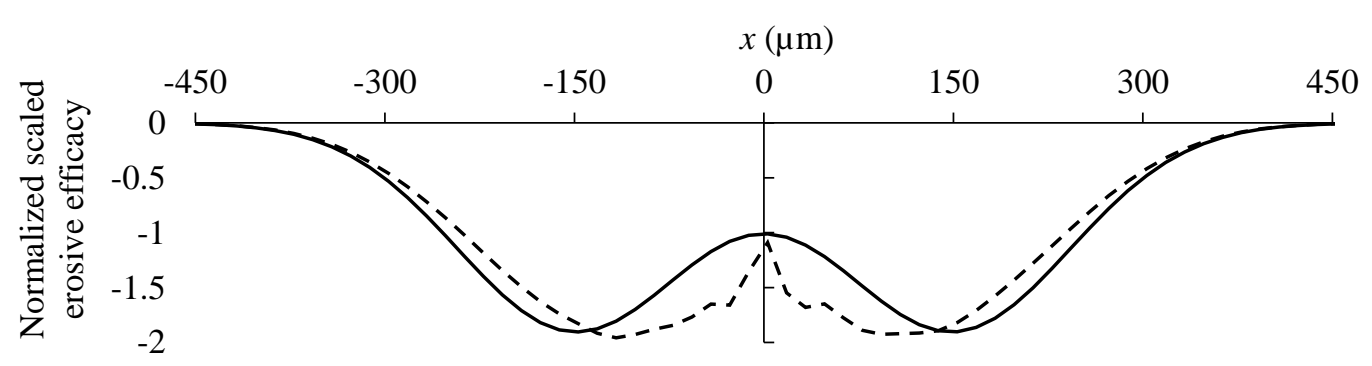

a)

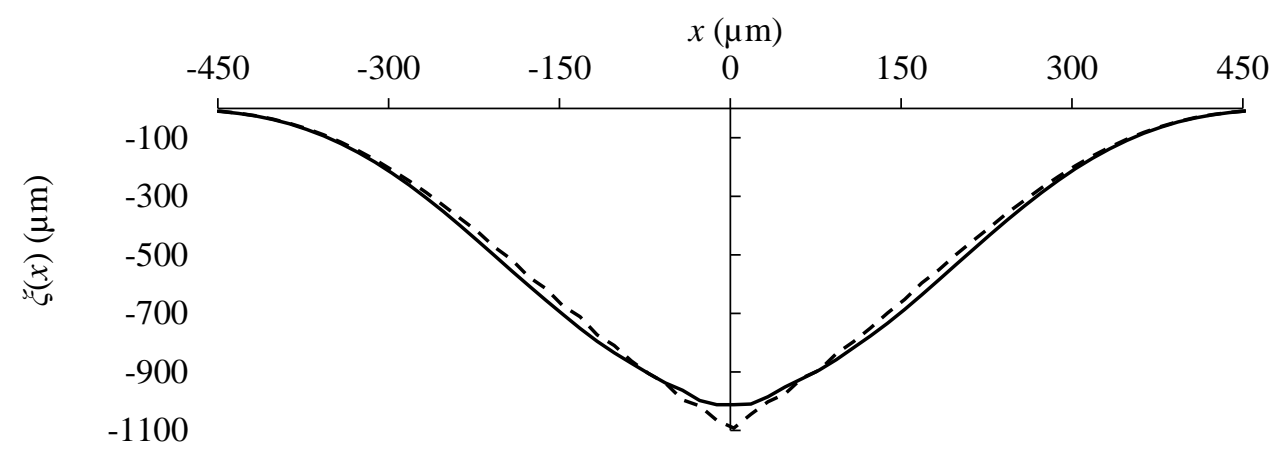

b)

Figure 2.9.1: (a) The original (solid line) and required (dashed line) normalized scaled erosive efficacies scaled by the number of repetitions predicted to evolve to the $V$-shaped target. (b) The predicted surface 
after using $E_{o}(x)$ in Equation 2-3. after $n_{f}=50$ repeats, evolved under the original $E_{r}$ (solid line) and the predicted $E_{r}$ (dashed line).

\subsubsection{Different Initial Guesses Leading to the Same $E_{r}$}

As a demonstration of the uniqueness of the predicted $E_{r}(x)$, a semicircle of radius $800 \mu \mathrm{m}$ was chosen as the desired profile, and three vastly different initial guesses of erosive efficacy were used in the algorithm (Figure 2.9.2a), the first a rectangular pocket of $80 \mu \mathrm{m}$ depth, and the other, as given by Equation 2-6 and finally the sum of two trigonometric functions $-30_{\mu m}+20_{\mu m} \cdot \sin \left(\frac{\pi}{900_{\mu m}} x-\pi\right)+10_{\mu m} \cdot \cos \left(\frac{2 \pi}{900_{\mu m}} x\right)$. The results were scaled by multiplying by the maximum depths of the profiles. Figure 2.9.2b demonstrates how the three predictions of the scaled erosive efficacies were close to one another (only $1.7 \%$ absolute average difference throughout the cross-section).

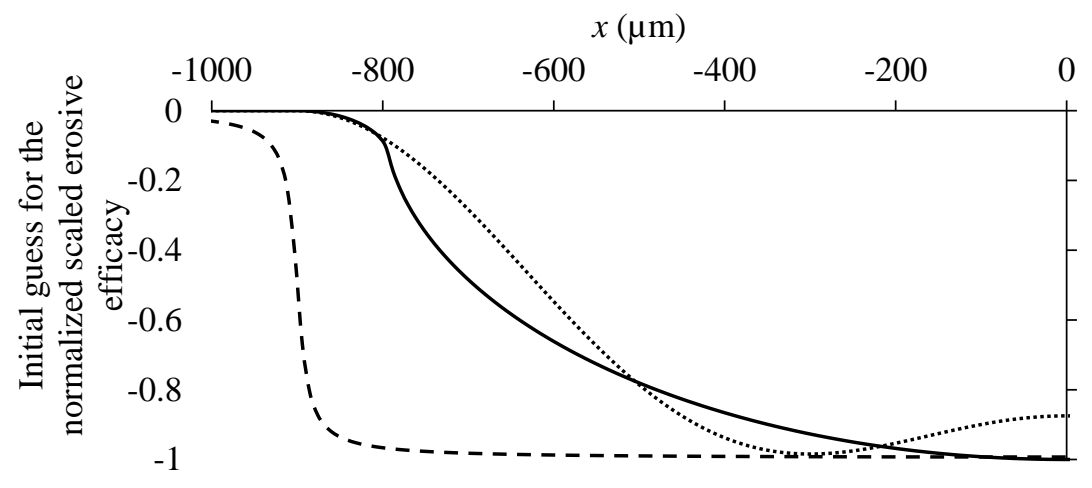

a)

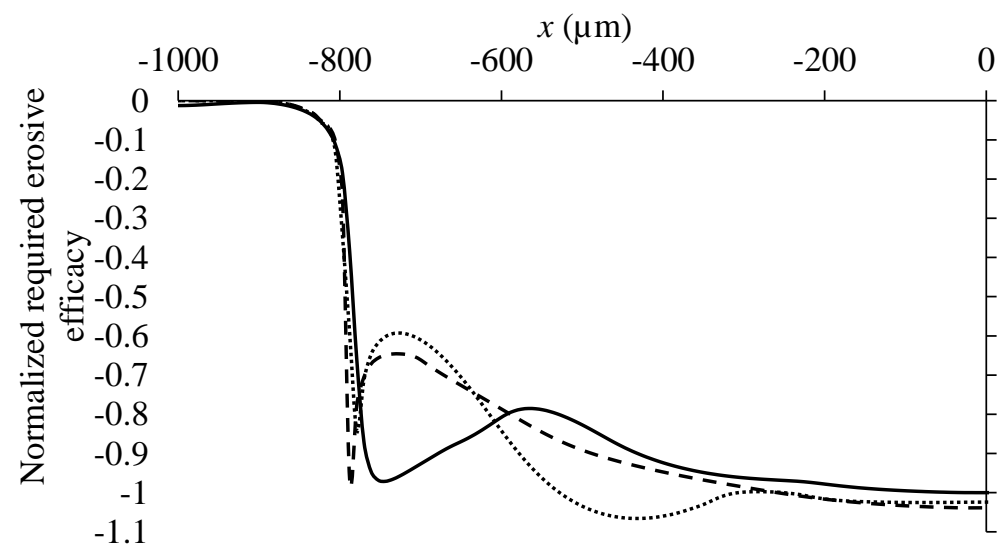

b)

Figure 2.9.2: (a) Different initial guesses (the dashed line represents a rectangular pocket with rounded edges, the dotted line a sinusoidal curve and the solid line the linearly scaled target) used as inputs for the inverse algorithm; (b) The predicted required scaled erosive efficacies $E_{r}(\mathbf{x})$ scaled by the number of repeats and divided by the target's maximum depth (the dashed, dotted and solid line correspond to rectangular, sinusoidal and linearly scaled initial guesses seen in (a)) 
The spikes and discrepancies near the edge of the $E_{r}(x)$ for the algorithm using a rectangular pocket as the initial guess could be explained to have been caused by the second derivative not existing (which was required for the smoothness term) in the vicinity of that point in the erosive efficacy which, according to Equation 2-3 would cause an inaccurate prediction in the erosion rate. This would, in turn, cause the algorithm to overshoot the correction for the next iteration. This would cause an inaccurate prediction in the erosion rate which would, in turn, cause the algorithm to overshoot the correction for the next iteration. Nevertheless, Figure 2.9.2b demonstrates the closeness of the predictions of the scaled erosive efficacy $(0.62 \%$ absolute average difference in depth).

\subsubsection{Leveling the Inverted Desired Profile Using the Same $E_{r}$}

In another effort, uniqueness was investigated in a limited fashion by finding the algorithm's prediction i.e. $E_{r_{1}}(x)$ and $n_{f}$, to machine a semicircular profile of $500 \mu \mathrm{m}$ radius, $D(x)$ (as Figure 2.9.3a depicts). Also considered was the $E_{r_{2}}(x)$, a hypothetical scaled erosive efficacy required to erode away a protrusion of inverted topography about the free surface, i.e. $-D(x)$. Finally, the two $E_{r}(x)$ s were compared to see if the same scaled erosive efficacy could level the protrusion to a flat surface after the same number of repeats with one additional repeat.

Figure 2.9.3a demonstrated the surface after a shallow erosion by $E_{r 1}(x)$ (dotted line), the predicted surface evolved in the glass after $n_{f}$ repeats $\xi_{1}\left(x, n_{f}\right)$ (dashed line) and the desired $D(x)$ (solid line); while Figure 2.9.3b depicts the initial surface $-D(x)$ (solid line), which was eroded with a constant scaled erosive efficacy $E_{r_{2}}(x)$ (in this illustration it was identical to $E_{r 1}(x)$ ), which after $n_{f}$ repeats left the predicted remains of the semicircular bump (dotted line).

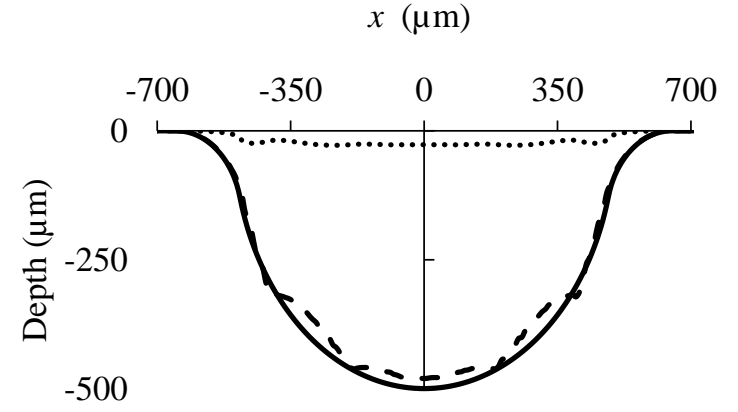

(a)

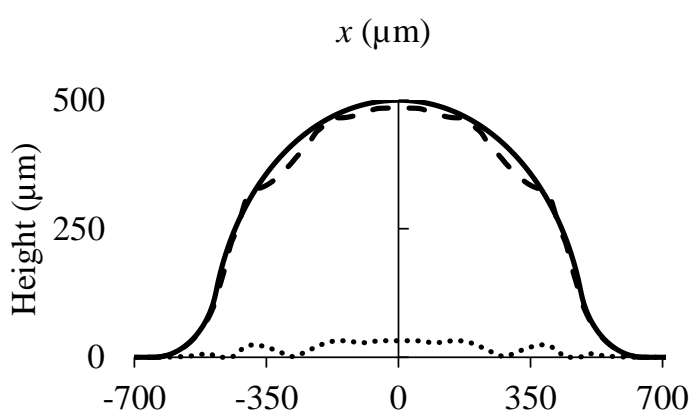

(b)

Figure 2.9.3: (a) evolution of a brittle surface (glass) given the dotted profile as erosive efficacy (b) erosion of a protrusion of mirrored topography given the desired mirror profile in solid line, the assumed initial surface with dashed line and the final eroded surface after $n_{f}$ repeats with dotted line machined in glass.

Preliminary predictions in Figure 2.9.3 suggested that the same $E_{r}(x)$ s used to erode the recessive, would level the protrusion. Yet, it remained to be proven if this true for any $D(x)$.

To inspect the example of the $500 \mu \mathrm{m}$ semi-circular target, the two $E_{r}(x)$ s could be more accurately compared in Figure 2.9.4a. Although the local difference was large at certain regions, 
the overall average difference in depth was only $5.485 \mu \mathrm{m}$, i.e. $19.5 \%$ of the shallow's depth and $1.1 \%$ of the target semicircle's depth. The small deviation could be attributed to the approximations made between the evolved surface and the desired profile. To demonstrate this point further, the location of the bump to be leveled could be seen in Figure 2.9.4b which was reasonably flat (yielding only an average depth of $0.6 \mu \mathrm{m}$ ) compared to the original bump:

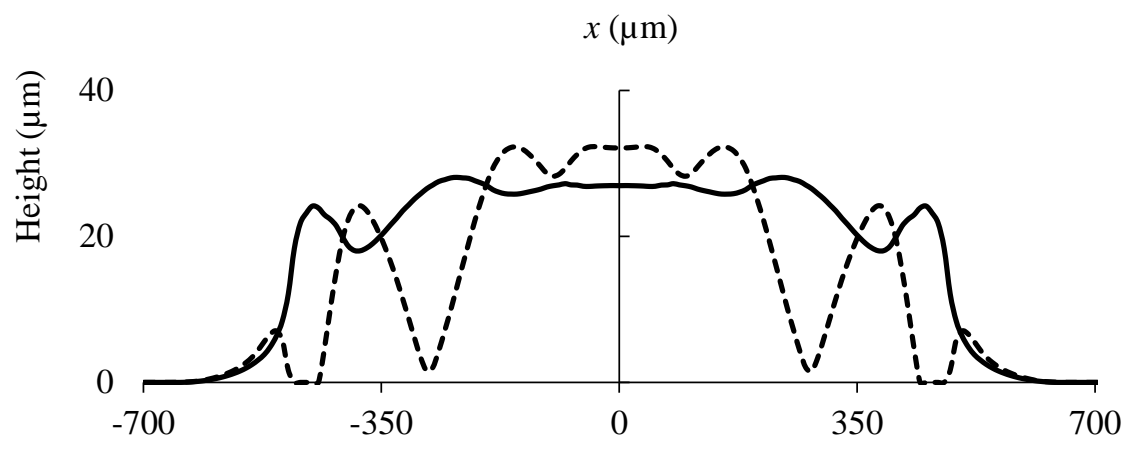

a) $x(\mu \mathrm{m})$

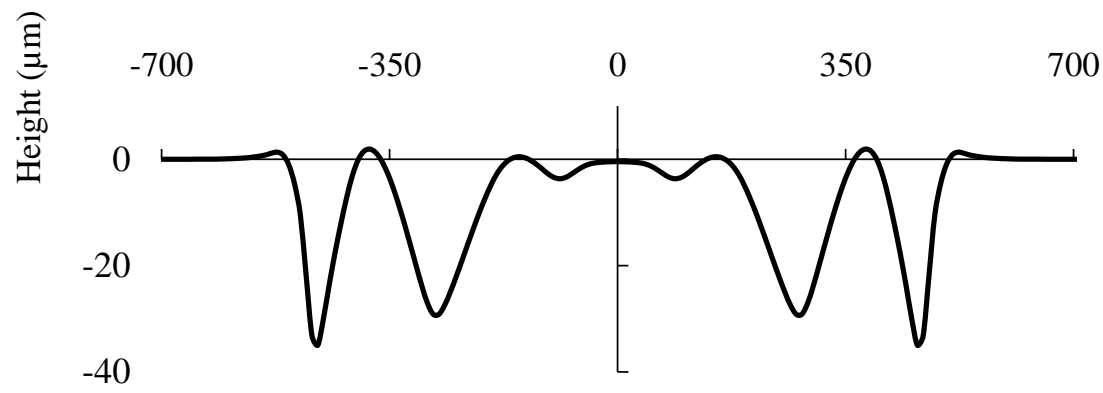

b)

Figure 2.9.4: (a) comparison between the evolved surface of the mirrored profile in dashed line and the predicted erosive efficacy mirrored about the zero line of the surface shown with a solid line. (b) The location of the leveled bump after $n_{f}+1$ number of repeats given the same scaled erosive efficacy

\subsection{Direct Application of Optimization to High A.R. Channels}

Inspired by Equation 2-12, previously introduced by Sookhak Lari and Papini [5], which was used to optimize the sources' locations and strengths to create a shallow profile (shallow profiles were defined in section 2.4.), optimization of the same parameters was investigated using surface evolution models (Equations 2-3 and 2-4) so that the evolution of $S(x)$ to $\xi\left(x, n_{0}\right)$ would be closer to $D(x)$. However, this required a simpler method to solve Equation 2-3 than the previously employed Method of Lines (MOL) using Mathcad ${ }^{\circledR}$ to find $\xi(x, n)$.

To this end, only symmetric targets were studied and both the time and space partial derivatives in Equation 2-3 were replaced with their respective Finite Difference (FD) counterparts: 


$$
\left\{\begin{array}{c}
\xi(x, n+\Delta n)=\xi(x, n)+\Delta n \cdot S(x) \cdot\left(1+\left(\xi^{\prime}(x, n)\right)^{2}\right)^{-\frac{k}{2}}\left(1-\in \cdot \frac{\left(\xi^{\prime \prime}(x, n)\right)^{2}}{\left(1+\left(\xi^{\prime}(x, n)\right)^{2}\right)^{\frac{3}{2}}}\right) \\
\xi^{\prime}(x, n)=\frac{\xi(x+\Delta x, n)-\xi(x, n)}{\Delta x} \\
\xi^{\prime \prime}(x, n)=\frac{\xi(x+2 \Delta x, n)-2 \xi(x+\Delta x, n)+\xi(x, n)}{\Delta x^{2}}
\end{array}\right.
$$

where $S(x)$ would be described by Equation 2-11, $\Delta n$ was the mesh size used to discretize $n$ (a number between 1 and $n_{0}$ ), $\Delta x$ was that used to discretize $x$.

To demonstrate the validity and the accuracy of replacing MOL with FD surface evolution, the erosion of a borosilicate sample by alumina was measured under a single repeat of a single source with scan speeds of $1 \mathrm{~mm} / \mathrm{s}, 2 \mathrm{~mm} / \mathrm{s}, 8 \mathrm{~mm} / \mathrm{s}$ and $16 \mathrm{~mm} / \mathrm{s}$ using the non-contact profilometer. These measurements were compared with the predictions for the surface evolution (under an $E_{j}(x)$ moving with $16 \mathrm{~mm} / \mathrm{s}$ for the number of repeats prescribed in Equation 2-5) by both MOL and FD, in Figure 2.10.1, which showed close agreement (and even improvement in accuracy when using FD). However, the repeat mesh had to be much finer and adjusted for each different $n$ ( $\Delta n$ used in FD was in the range [0.08,0.09] while $\Delta n$ s as coarse as 0.889 caused no significant change in MOL predictions):

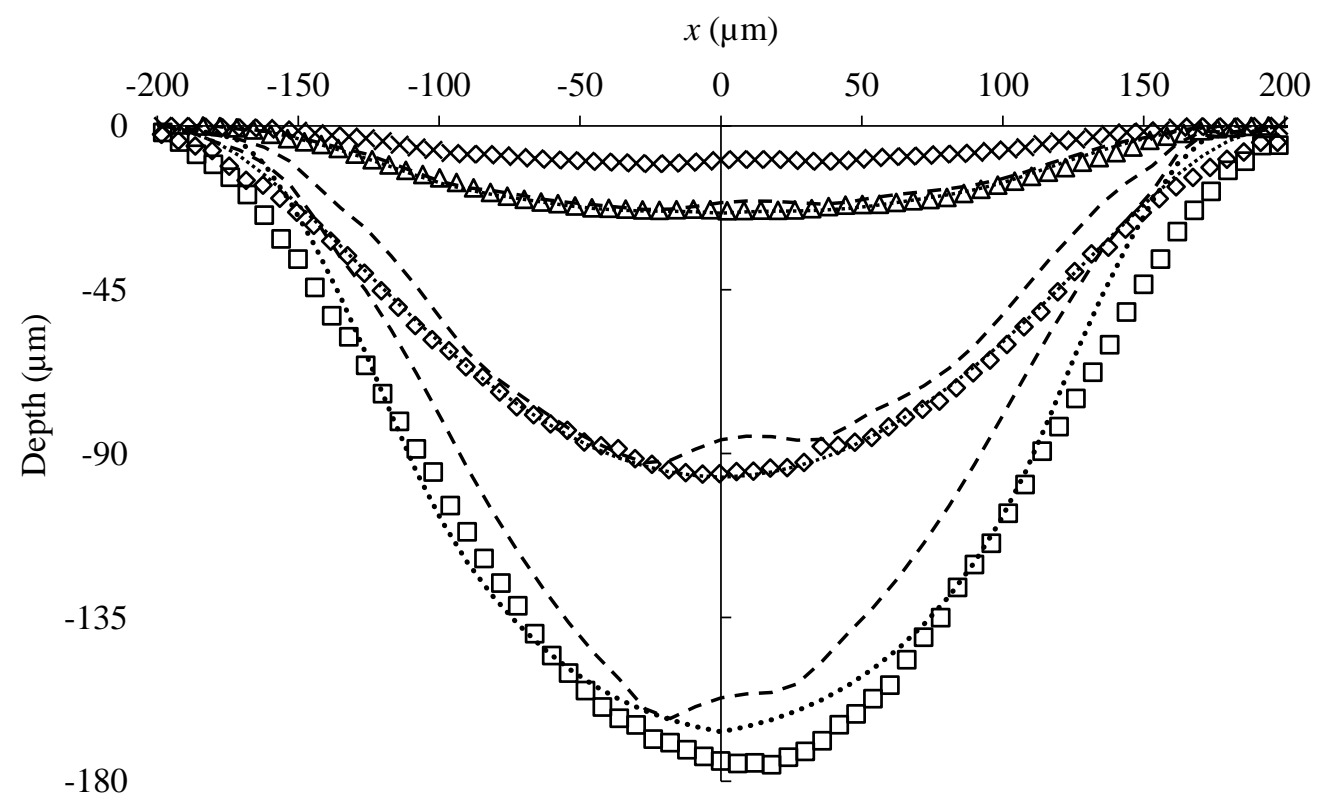

Figure 2.10.1: Comparison between the experimental results (markers $\times$ for $16 \mathrm{~mm} / \mathrm{s}, \Delta$ for $8 \mathrm{~mm} / \mathrm{s}$, $\diamond$ for 2 $\mathrm{mm} / \mathrm{s}$ and $\square$ for $1 \mathrm{~mm} / \mathrm{s}$ ) and surface evolution predictions by MOL (shown with the dashed lines) and FD (shown with the dotted lines) 
Having established the reliability of an FD based surface evolution prediction, the objective function, $R_{\chi}$, to be minimized was:

$R_{\chi}(x)=\frac{\int\left|\xi\left(x, n_{0}\right)-D(x)\right| d x}{W}$

where $\xi\left(x, n_{0}\right)$ was the predicted location of the surface after the predetermined number of repeats $n_{o}, D(x)$ the profile of the target, $W$ the range between its two edges (as defined previously by Nouhi et al. [48]), or its width.

Hence, for 26 hypothetical sources evenly distributed about $x=0$ the location of the sources, $\left(x_{0}\right)_{\kappa}$, and their scan speed, $\left(v_{s}\right)_{\kappa}$, were optimized using the General Reduced Gradientnonlinear (GRG)[78] method available in Excel® (Version 2015, Microsoft Inc., 1 Microsoft Way Redmond, WA 98052, US) which were bound by the conditions seen in Equation 2-15:

$\forall \kappa \in[1,13]:\left\{\begin{array}{c}\left(x_{0}\right)_{\kappa} \leq\left(x_{0}\right)_{\kappa+1} \\ -\frac{W}{2} \leq\left(x_{0}\right)_{\kappa} \leq \frac{W}{2} \\ v_{\max } \leq\left(v_{s}\right)_{\kappa} \\ \exists l \in[13,26]:\left(x_{0}\right)_{l}=-\left(x_{0}\right)_{\kappa}\end{array}\right.$

where the first and second conditions were applied, only to reduce the range within which $\left(x_{0}\right)_{\kappa}$ was to be found, the third condition to ensure the scan speed necessary for the operation would not exceed that of the stage used for AJM machining (and also prevent prediction of zero scan speeds) and the fourth ensured that the predicted scaled erosive efficacy would be symmetric, as was the target.

Optimization was not applied to the ductile target (PMMA) due to error propagation which would have resulted from using finite difference approximation for partial derivatives.

\subsection{Inherent Tendency to Form Wedge-Shaped Profiles in Brittle Materials}

Symmetric channels in glass formed by a single source tracing a straight path naturally evolve to form V-shaped (symmetric wedge) channels with relatively constant, shallow-sloped walls [65]. According to Equation 2-3, a linear $E_{r}(x)\left(\partial E_{r}(x) / \partial x=\right.$ constant $)$ results for regions away from the locations at which the slope changed, and the eroded surface would thus propagate linearly.

It was therefore hypothesized that non-symmetric wedges would be relatively easy to machine in brittle materials, i.e., that the inverse algorithm would rapidly converge to a required erosive efficacy given the desired wedge profile. Indeed, Figure 2.11.1b shows that the evolved surface, as predicted by Equation 2-3, approached the desired asymmetric wedge profile, $D(x)$, 
after only $n=10$ iterations of the algorithm. Figure 2.11.1a shows that, as expected, the required efficacy also rapidly converges to a wedge shape. In summary, creating wedges and relatively shallow slopes wedge in the glass does not present a great challenge to the inverse algorithm because such shapes naturally evolve in the glass. Therefore, the focus of this work became the use of the inverse algorithm for the much more challenging case of generating curved profiles and features with steep sidewalls.

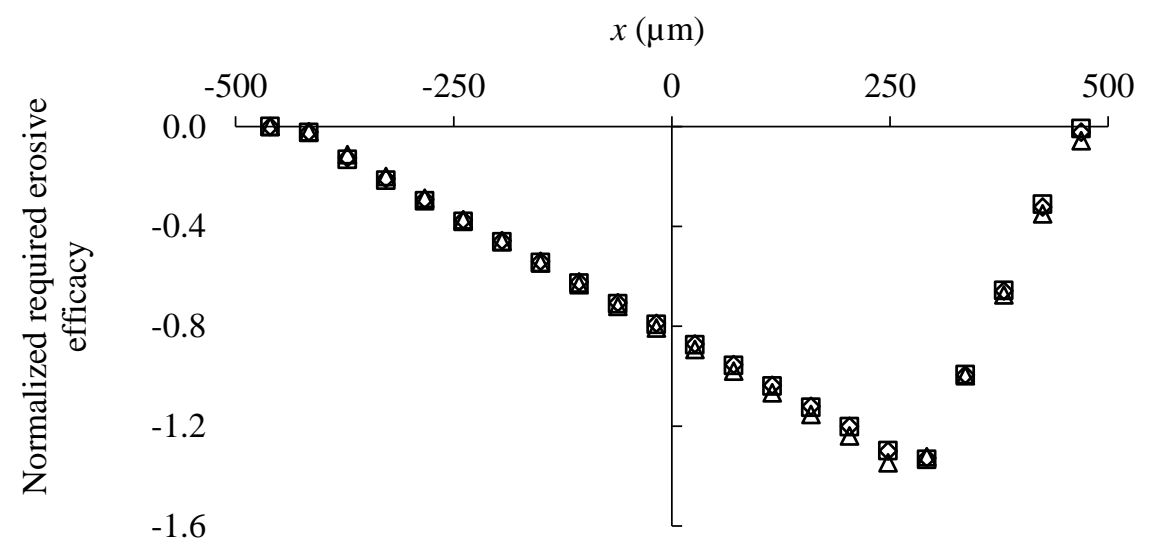

a)

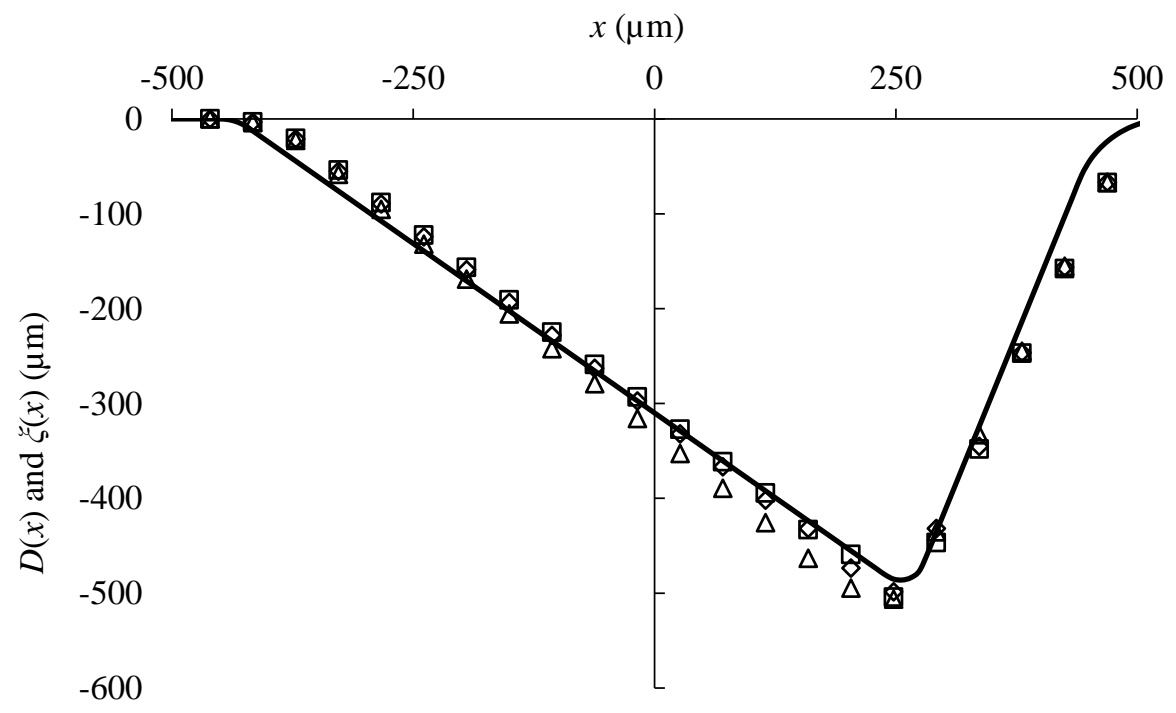

b)

Figure 2.11.1: (a) The required erosive efficacy $E_{i}(x)$ predicted by the inverse algorithm after $i$ iterations $(i=10$ represented by the triangular markers $(\Delta), i=50$ shown by the diamond markers $(\diamond)$ and $i=100$ iterations shown by the square markers $(\square)$ ) required to create (b) a particular desired $D(x)$ asymmetric wedge in glass, together with the evolved surfaces using $E_{i}(x)$. 


\subsection{Inherent Tendency to Form Curved Profiles in Ductile Materials}

Analogous to the natural tendency for surfaces to evolve with sloped sidewalls in brittle materials (Section 2.10), in ductile materials, the tendency is to produce more U-shaped channels, with curved bottoms [65]. It was thus hypothesized that curved surfaces would be relatively easy to produce in ductile targets. As expected, Figure 2.12.1a shows that even after only $i=10$ iterations, the general shape of the scaled erosive efficacy required to machine a $500 \mu \mathrm{m}$ radius circular cross-section has been defined, and Figure 2.12.1b shows that there is virtually no difference between the desired profile and predicted profiles at $i=10,50$ and 100 iterations. Therefore, the focus became the use of the inverse algorithm in the much more challenging case of generating sloped profiles in PMMA.

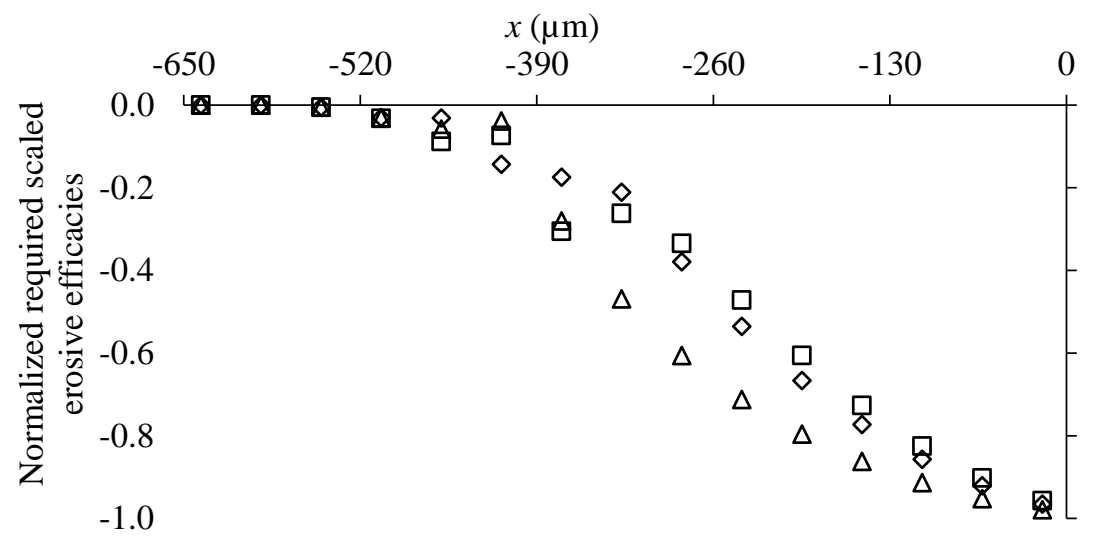

a)

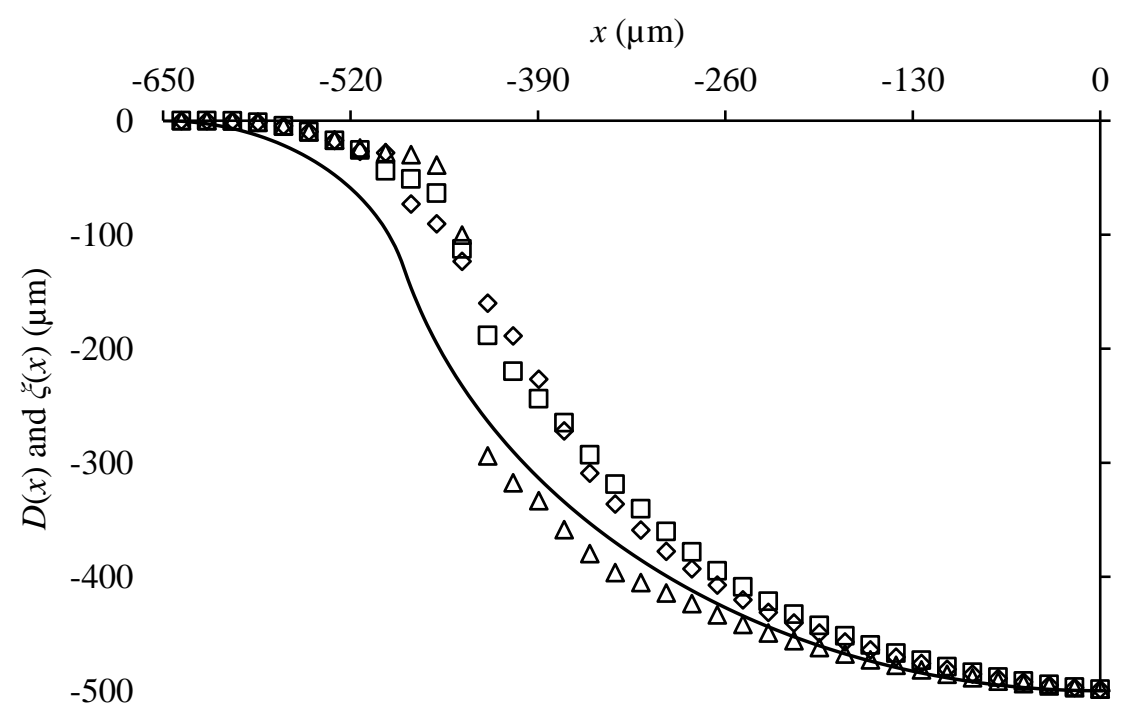

b)

Figure 2.12.1: (a) The scaled erosive efficacy $E_{i}(x)$ predicted by the inverse algorithm after $i$ iterations $(i=10$ represented by the triangular markers $(\Delta), i=50$ shown by the diamond markers $(\diamond)$ and $i=100$ iterations shown by the square markers $(\square)$ ) required to create (b) a desired $D(x) 500 \mu \mathrm{m}$ radius circular cross-section in PMMA, together with the evolved surfaces using $E_{i}(x)$. 


\subsection{Evaluation of Model}

With the theoretical approach established to predict the required parameters to machine a desired profile of high AR using AJM, the proposed method was to be evaluated for features that were not previously, as indicated in sections 2.11 and 2.12. The inverse method's predicted $E_{r}(x)$ was used in the surface evolution equations, the predicted profiles of which were compared to $D(x)$. Additionally, the prediction of the GRG non-linear in section 2.10 was compared in accuracy to the inverse methods' results.

\subsubsection{Channels in Glass}

The targets to be evolved in the glass were chosen as concave (extruded) semicircles with radii between $500 \mu \mathrm{m}$ and $900 \mu \mathrm{m}$ with rounded edges and convex (protruding) semicircles of radii $600 \mu \mathrm{m}$ and $800 \mu \mathrm{m}$ within a trapezoidal pocket of depths $600 \mu \mathrm{m}$ and $800 \mu \mathrm{m}$, respectively. The later targets were chosen to demonstrate the application of the algorithm in controlling the curvature of features that had their center of curvature outside of the channels. The two convex profiles were chosen due to the promising results of their concave counterparts.

Table 2-1, column 2 shows the desired, $D(x)$, semi-circular cross-sections of various radii, together with the corresponding predicted evolved profiles $\xi\left(x, n_{f}\right)$ resulting from the application of the inverse technique that predicted the required scaled erosive efficacy $E_{r}(x)$. In all cases, the fit is close, with an absolute average difference in depth between $D(x)$ and $\xi\left(x, n_{f}\right)$ below $8 \%$ of the radius. Table 2-1, column 2's last two entries depict half the profile of the predicted surfaces as a result of erosion under $E_{r}(x)$ predicted by the GRG-nonlinear optimization and the inverse algorithm for a $600 \mu \mathrm{m}$ semicircle protrusion and an $800 \mu \mathrm{m}$ protrusion. The absolute average difference for the optimization from the target was $5.3 \%$ and $3.2 \%$ for $600 \mu \mathrm{m}$ protrusion and the $800 \mu \mathrm{m}$ protrusion, whereas the inverse algorithm's prediction deviated from the target by $3.4 \%$ and $1.8 \%$ respectively.

The high agreement between the predicted profiles, using the algorithm's $E_{r}(x)$, and the desired was evidence of the algorithm's competence but also facilitated by avoiding the approximation applied in the direct optimization of sources' distribution (the GRG-nonlinear). 
Table 2-1: Column 1: the predicted required scaled erosive efficacy $\left(E_{r}(x)\right)$ for dashed line, the GRGnonlinear's $S(x)$, dotted line, inverse compared to the initial guess $\left(E_{0}(x)\right)$ given by Equation 2-6, solid line; column 3: the evolved $\xi(x, n)$ from repeating $E_{r}(x)$ for dashed line, from repeating GRG-nonlinear's $S(x)$, dotted line, compared to $D(x)$, by a solid line and cross markers.

\begin{tabular}{|c|c|c|}
\hline $\begin{array}{l}\text { TargetProfil } \\
\text { e }\end{array}$ & Scaled erosive efficacy & Predicted evolved surface and the target \\
\hline 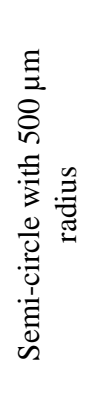 & 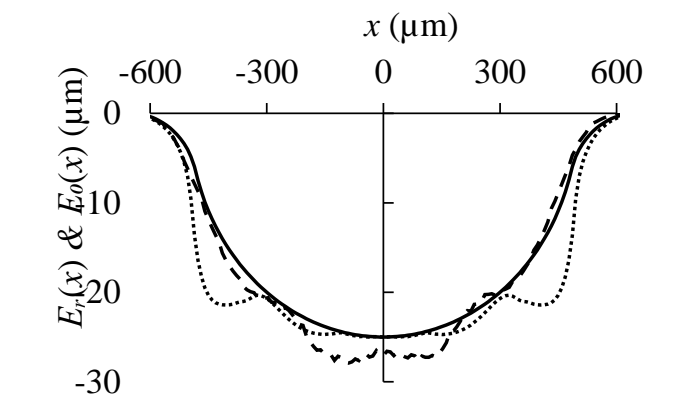 & 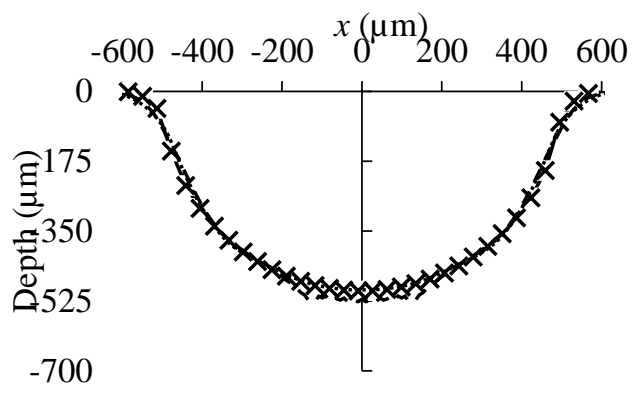 \\
\hline 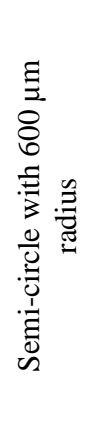 & 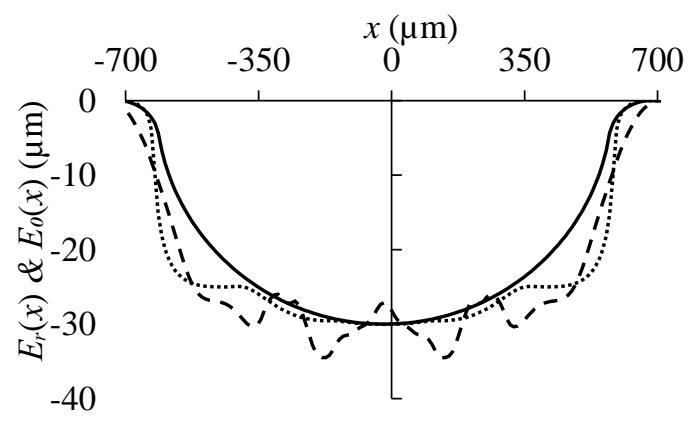 & 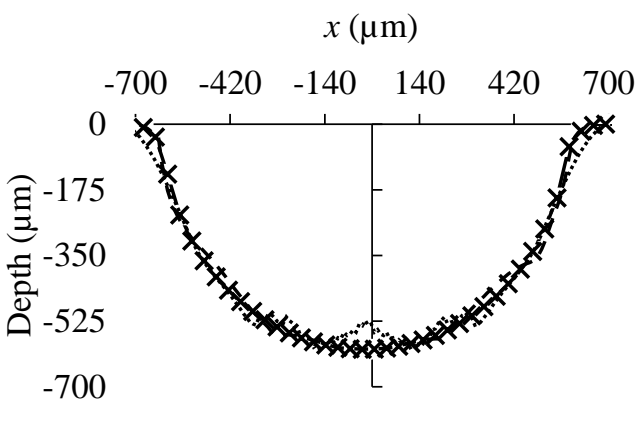 \\
\hline 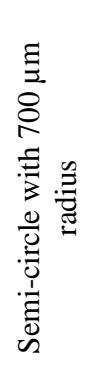 & (1) & 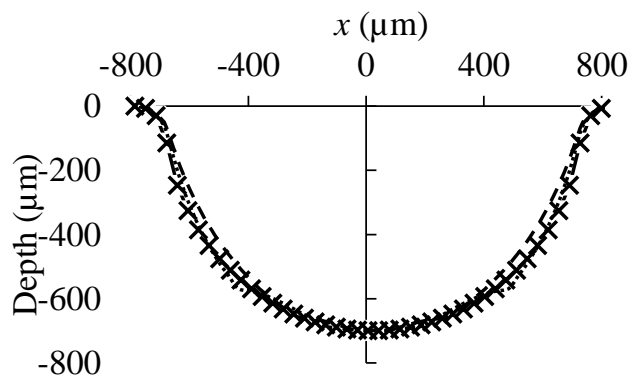 \\
\hline 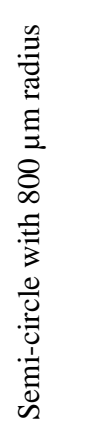 & 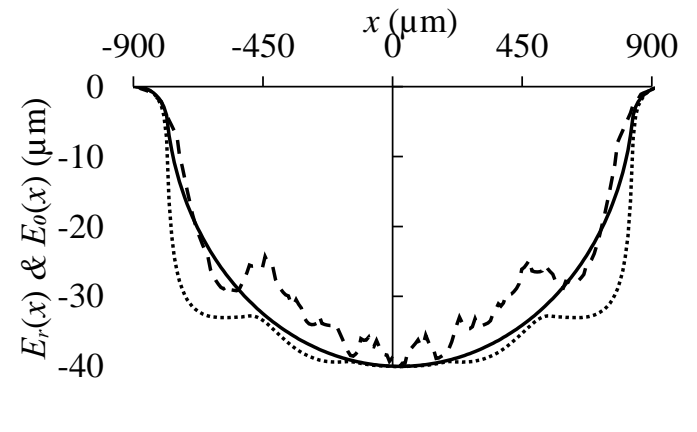 & 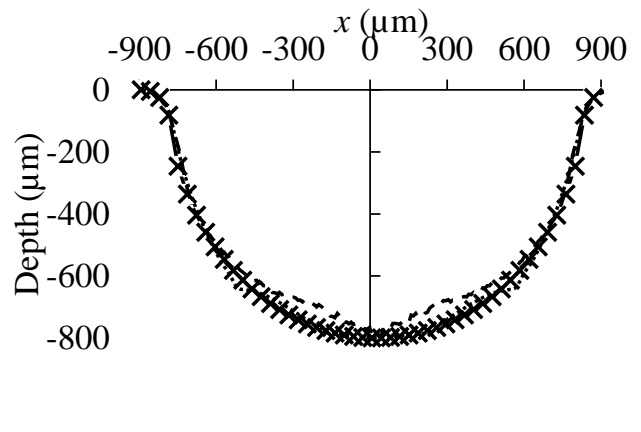 \\
\hline
\end{tabular}




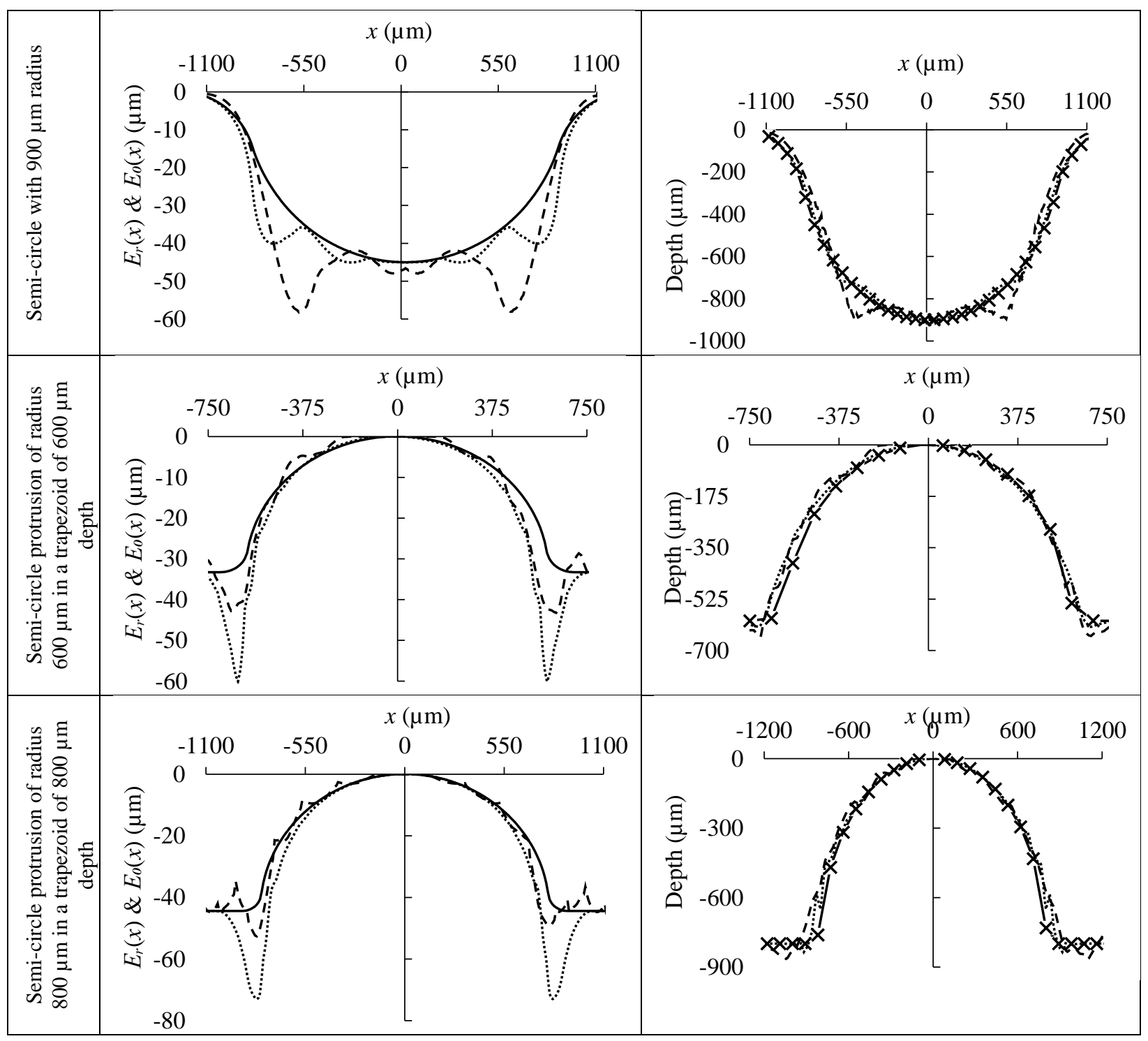

Therefore, the deviation from $D(x)$ was larger for the GRG-nonlinear optimization. This was mainly due to the inevitable presence of sharp cusps if the mesh size was so small that the depth's rate of change became exceedingly large. A case study for the appropriate mesh size demonstrated that, even though only plausible predictions was possible for the output of the optimization, the intermediate iterations (which were considered for the variables path to minimize the deviation) could not individually be monitored in an automated optimization package that resulted in the presence of cusps. Hence, the inverse methodology was more robust to the high slopes compared to the GRG-nonlinear.

Moreover, unlike the inverse algorithm, the GRG-nonlinear method (directly optimizing the machining parameters) showed significant dependency on the initial guess for $E_{r}(x)$ (the independence of the inverse algorithm from $E_{0}(x)$ was established in section 2.9). Also, the computation cost for generating $E_{r}(x)$ using optimization was in average three times that of using the inverse method, making the latter more efficient by comparison. 


\subsubsection{Channels in PMMA}

To test the limits of application of the inverse algorithm to ductile materials and the ability to machine irregular targets, a symmetric ramp with a $45^{\circ}$ incline and two asymmetric ramps having $30^{\circ}$ and $45^{\circ}$ incline in PMMA were used. The edges of the target at which the slope would have been discontinuous were rounded according to section 2.7.

The result of applying the inverse algorithm to PMMA can be seen in Table 2-2 to closely agree with the desired (the average difference in depth was less than $0.4 \%$ of the target's depth). The first column of Table 2-2 demonstrates that the required $E_{r}(x)$ did not have a linear relation with $D(x)$.

Table 2-2: Column 1: the predicted required scaled erosive efficacy $\left(E_{r}(x)\right)$ for dotted line, inverse compared to the initial guess $\left(E_{0}(x)\right)$ given by Equation 2-4, solid line; column 3: the evolved $\xi(x, n)$ from repeating $E_{r}(x)$ for dotted line, compared to $\mathrm{D}(\mathrm{x})$, by a solid line and cross markers.

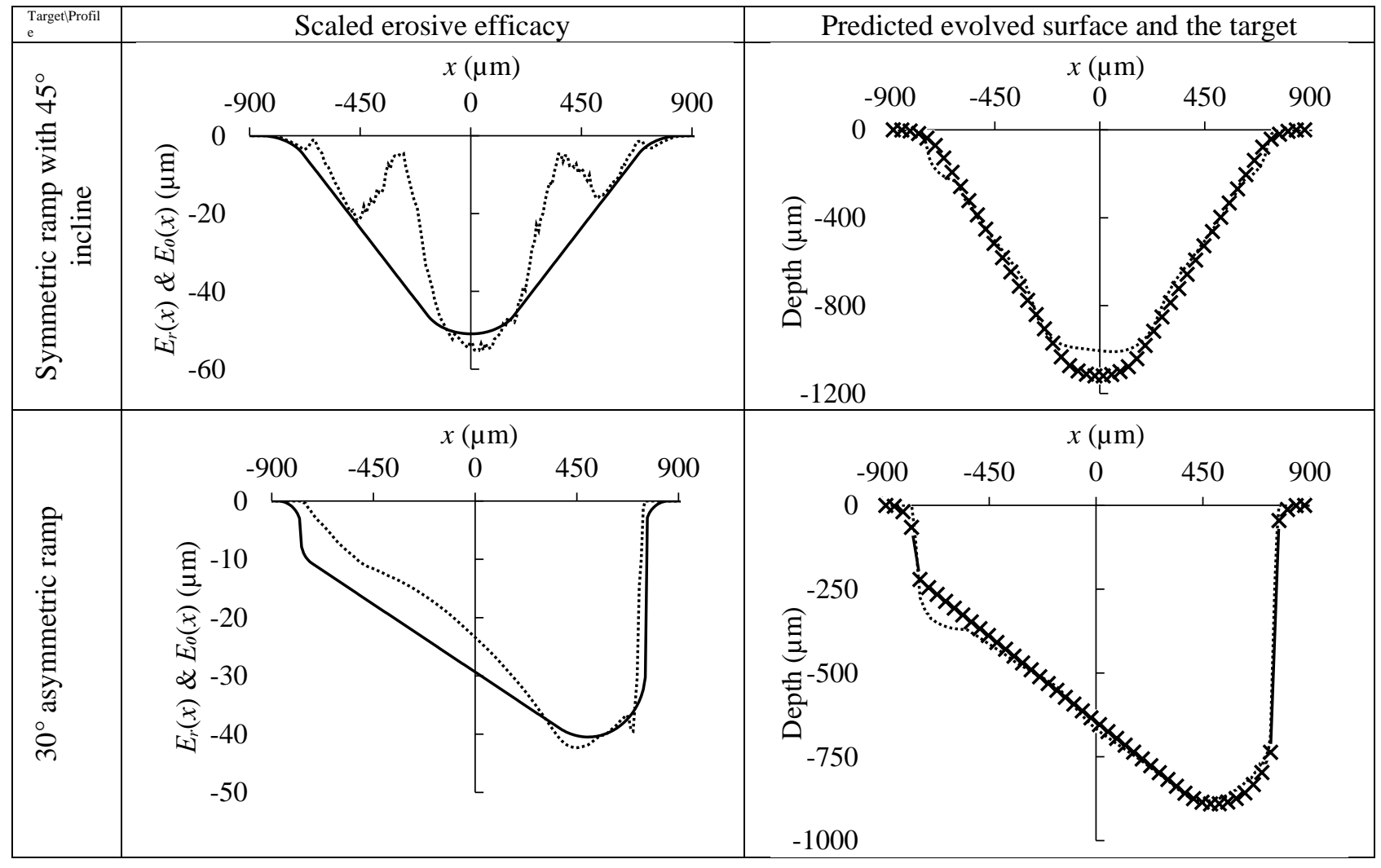




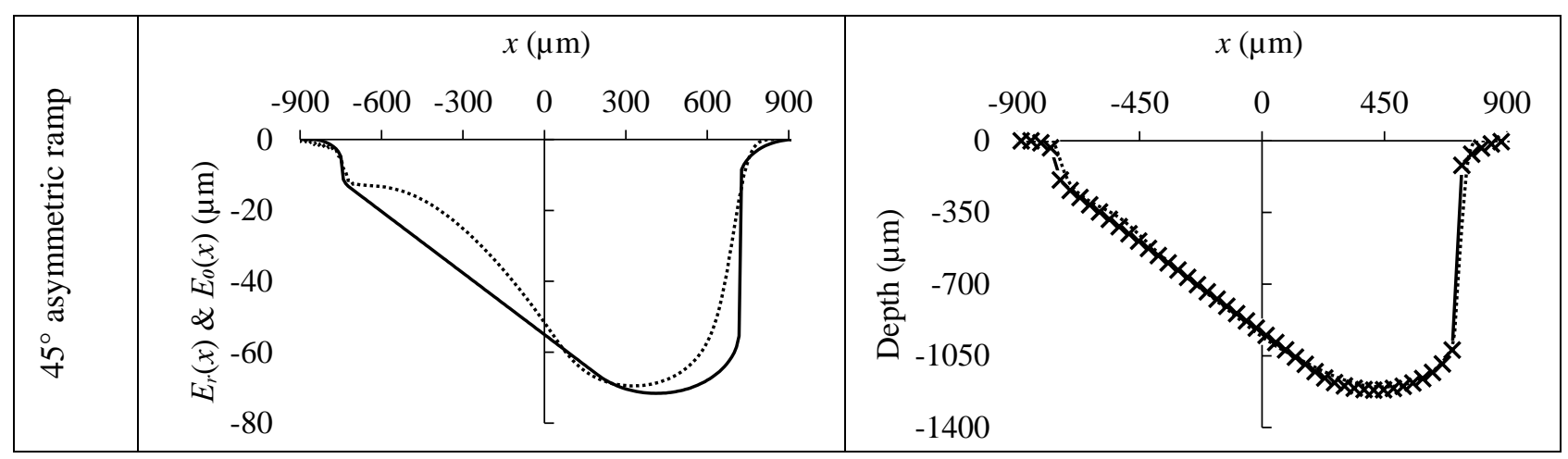

Even though, the evolution of $E_{r}(x)$ closely matched the targets, calculation of $\xi(x, n)$ required trial-and-error to determine the value of $\in$ to avoid sharp cusps, e.g. the $30^{\circ}$ asymmetric ramp's over-prediction of depth in the vicinity of the right edge resulted in noise in the values of $E_{r}(x)$. However, the abnormality (caused by the over-prediction of depth at few nodes) was smoothened when applying Equation 2-4. Thus, care needed to be taken when applying the inverse algorithm to ensure a realistically smooth $\xi(x, n)$ in every iteration of the method. This made Equation 2-4 unsuitable for integration with an optimization method.

Moreover, the dotted line in Table 2-2's depiction of the symmetric ramp's $\xi\left(x, n_{f}\right)$ is deeper than $D(x)$ close to the sidewalls. To elaborate, even though $D(x)$ may be desired to have a less steep slope in the vicinity of the edge, the evolution of PMMA would not allow that and would rapidly evolve, causing $E_{r}(x)$ to be corrected so that any fluctuations in depth occurring outside the bounds of $D(x)$ 's width were reduced to zero, which would clearly be an incorrect prediction. To eliminate this deviation, the asymmetric ramps were offset by at least $200 \mu \mathrm{m}$ near each edge. A higher no would require a higher offset.

Moreover, the algorithm adjusted the required machining parameters independent of the surface evolution model (e.g. the models for brittle and ductile erosion were drastically different), suggesting that it could possibly be employed to machine features in processes similar to AJM.

All features processed through the algorithm thus far have been two dimensional. However, hypothetically, this method could be used to control not only the $2 \mathrm{D}$ cross-sectional profile but also 3D features machined. This may be done by AJM by breaking the feature in question into multiple desired profiles extracted from parallel sectioning planes, applying the inverse algorithm to each individual profile and optimizing a predetermined number of sources' route to match the required location and scan speed while transitioning from each prescribed source location to the next in an optimal path. 


\subsection{Summary}

The method presented in chapter 2 improved on the former inverse methods of machining brittle and ductile targets using adjacent passes in AJM demonstrating that the previous linear propagation assumption would not hold for high AR targets. Therefore, through the application of the algorithm in section $2.5 ., E_{r}(x)$ was adjusted and uniquely predicted for a brittle target so that $\xi(x, n)$ would approach $D(x)$.

The inverse algorithm was applied to semicircle targets in the brittle glass and linear ramps in the ductile PMMA to control the radius of curvature and the slope respectively, which, according to sections 2.11 and 2.12, opposed the inherent tendency of the targets' evolution. Every target required a smooth transition in slope which was achieved by rounding the edges as instructed by section 2.7 .

Furthermore, the predictions of the inverse method, for the brittle glass, were compared to those resulting from optimization of the sources' location $\left(x_{0}\right)_{j}$ and scan speed $\left(v_{s}\right)_{j}$ which proved the inverse method to be more computationally efficient and accurate than the GRG-nonlinear optimization.

Further study into practical requirements and machinability of the $E_{r}(x)$ predictions will be provided in chapter 3 of this thesis which will compare the machined profiles to case studies of several desired. 


\section{Implementation and Verification}

The following chapter is based on the following journal article (submitted):

Aria Ghazavi, Marcello Papini, (2018), An Inverse Method for the Abrasive jet micro-machining of High Aspect Ratio Channels of Desired Topography - Part II, Experiment

\subsection{Introduction}

Chapter 2 demonstrated that an inverse technique could be used to predict the required scaled erosive efficacy $E_{r}(x)$ required to machine high AR micro-channels with particular desired cross-sectional topographies, $D(x)$. The most challenging geometries were identified to be channels with a curved cross-sectional sidewall in brittle targets (e.g. borosilicate glass) and channels with linear sidewalls in a ductile target (e.g. PMMA). This section experimentally validates the inverse method by comparing the measured cross-sectional profiles resulting from machining using the predictions of the method to those desired initially, for a variety of these more challenging geometries.

\subsection{Experiments}

AJM experiments were performed in order to machine the following desired microchannel cross-sectional shapes in boro-silicate glass: a $500 \mu \mathrm{m}$ deep rectangular pocket, an 1100 $\mu \mathrm{m}$ wide and $300 \mu \mathrm{m}$ deep trapezoid, and $500 \mu \mathrm{m}$ to $900 \mu \mathrm{m}$ radius semi-circular. In addition, protruding semicircles of radii $600 \mu \mathrm{m}$ and $800 \mu \mathrm{m}$ were machined with sufficient distance from the sidewalls of the channel as well as a $600 \mu \mathrm{m}$ radius semi-circular protrusion $200 \mu \mathrm{m}$ below the free surface. In PMMA, the desired cross-sectional shapes were: a symmetric wedge of width $1600 \mu \mathrm{m}$ and depth $1200 \mu \mathrm{m}$, and asymmetric wedges having slopes of $30^{\circ}$ and $45^{\circ}$ up to the depth of $800 \mu \mathrm{m}$ and $1000 \mu \mathrm{m}$, respectively. To ensure that protrusions below the surface could also be machined, a semicircle of $600 \mu \mathrm{m}$ radius etched within an $800 \mu \mathrm{m}$ deep trapezoid was also successfully etched in the glass, such that the protrusion was $200 \mu$ below the surface at its highest point. As discussed in chapter 2 of this thesis, the chosen topographies represent the most challenging cases i.e., those that run counter to the natural tendency of surface evolution.

\subsection{Experimental Setup}

The experimental setup was identical to that described by Getu et al. [47]. An Acuflo AF10 blaster (MB 1005 Microblaster, Comco, Inc., Burbank, CA, USA) was used at a pressure of $400 \mathrm{kPa}$ to blast $25 \mu \mathrm{m} \mathrm{Al}_{2} \mathrm{O}_{3}$ powder through a rectangular $0.2 \mathrm{~mm} \times 2 \mathrm{~mm}$ nozzle (MB1500-20 Comco, Inc., Burbank, CA, USA). To improve the uniformity of the abrasive mass flow rate [77], the powder was mixed in the hopper using a rotary blade (Arrow Model 850, Arrow Engineering Co., Inc., Hillside, NJ, USA) and the air was dried using desiccant and a refrigeration air drier. The abrasive mass flow rate was recorded as the average of that measured before and after machining the channels by collecting and weighing (Sartorious CP224S, 
Mississauga, ON, Canada) the particles blasted into a can sealed with P100 filter for $100 \mathrm{~s}$. Consistent with earlier unmasked AJM studies [5], the target was placed at a very low $130 \mu \mathrm{m}$ standoff distance so that a relatively small $450 \mu \mathrm{m}$ wide machining scaled erosive efficacy resulted. The target movement was controlled by two linear actuators (Aerotech Inc., Pittsburgh, PA, USA) with a $0.5 \mu \mathrm{m}$ positioning accuracy in two perpendicular directions. During each machining experiment, the abrasive was sprayed on the target while the stage moved it under the jet in a raster motion. The targets were $3.2 \mathrm{~mm}$ thick borosilicate glass (Swift Glass, Elmira, NY, U.S.A.) and $3 \mathrm{~mm}$ thick Poly(methyl methacrylate) (McMaster Carr, Aurora, OH, US) plates, which erode in typically brittle and ductile fashions, respectively [47].

\subsection{Machining of Channels in Glass and PMMA}

For each of the desired shapes indicated at the beginning of Section 3.5, the inverse algorithm of Section 2.5. was used to predict the scaled erosive efficacies, $E_{r}(x)$ and the number of repeats $n_{f}$, required to evolve the surface to approximate the desired surface $D(x)$. Then, the sources' optimization, previously introduced by Sookhak Lari et al. [5], was used to determine the machining procedure required to produce an approximation, $S(x)$ to the required $E_{r}(x)$. These procedures involved performing adjacent passes at various offsets $\left(x_{0}\right)_{\kappa}$ and speed, $\left(v_{s}\right)_{\kappa}$ and then repeating them $n_{f}$ times to obtain the final measured profile. The machining procedures to produce $S(x)$ are provided in the appendix.

\subsection{Results and Discussion}

Glass tends to erode in a brittle manner, such that the erosion is maximized when particles are incident perpendicular (Figure 3.5.1), and minimized when incident at oblique angles. PMMA erodes in a ductile fashion that maximizes erosion at oblique impact and minimizes it a perpendicular incidence (Figure 3.5.1). The changing topography of the channels as they become deeper results in changes in the instantaneous particle strike angles along the cross-sections. As will be seen, this interplay between local impact angle and differing erosion behavior largely determines the predicted scaled erosive efficacy required to produce a given shape in these materials. The predicted and approximated scaled erosive efficacies, $E_{r}(x)$ and $S(x)$, respectively, required to produce the various desired $D(x)$ cross-sectional shapes are shown in the part (a) of Figure 3.5.1.1 to Figure 3.5.1.8, Figure 3.5.1.10 to Figure 3.5.2.1 and Figure 3.5.2.3 to Figure 3.5.2.6 part (b) of these figures show the desired, $D(x)$, predicted $\xi(x)$ (i.e. using $S(x)$ in the surface evolution equations), and measured micro-channel profiles. 


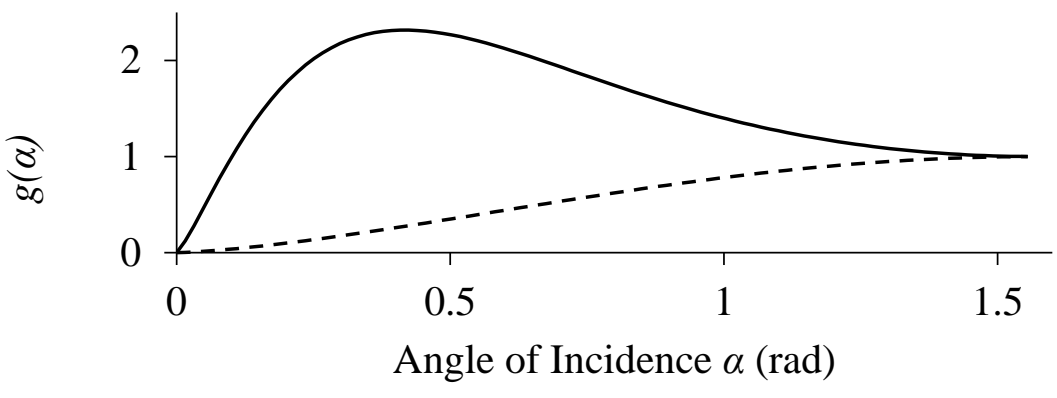

Figure 3.5.1: Dependence of erosion rate on the angle of attack for glass (dashed line) [64] and PMMA (solid line) [35] described by $g(\alpha)$ as discussed in chapter 2.

\subsubsection{Channels in Glass}

The following shapes were machined in borosilicate glass provided by the manufacturer named in section 3.3. The justification for why these specific shapes were chosen could be found in section 2.11. Every desired profile was modified for its minimum radius of curvature not to be lower than $100 \mu \mathrm{m}$ as discussed in section 2.8. (except for the hypothetical profile discussed in section 3.5.1.1.).

\subsubsection{Rectangular - Limitation of Maximum Sidewall Slope}

It was initially desired to machine a $500 \mu \mathrm{m}$ deep rectangular pocket in the glass. However, the inverse algorithm yielded a scaled erosive efficacy (Figure 3.5.1a) which, when used in the surface evolution equation, yielded a more trapezoidal shape (Figure 3.5.1b). It was not possible to create a vertical sidewall in the glass using an adjacent pass raster technique of previously discussed in Section 2.11, because the maximum slope at the periphery of the feature was limited by the finite slope of the source's scaled erosive efficacy itself, and the nature of the brittle erosion process which meant (Figure 3.5.1) that the flat centerline (perpendicular incidence) would always erode more rapidly than the sidewalls. In other words, regardless of the arrangement of the individual sources, the peripheral shape of the machined feature will always depend on the shape of the scaled erosive efficacy.

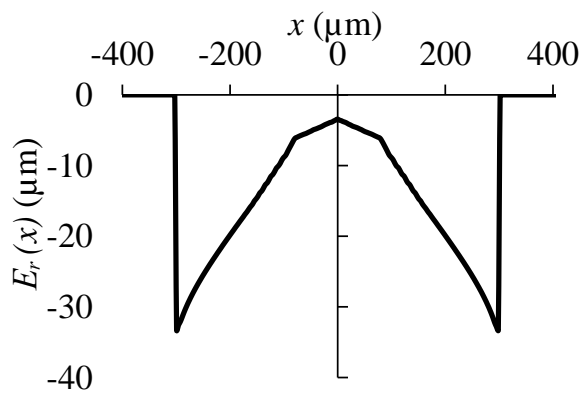

(a)

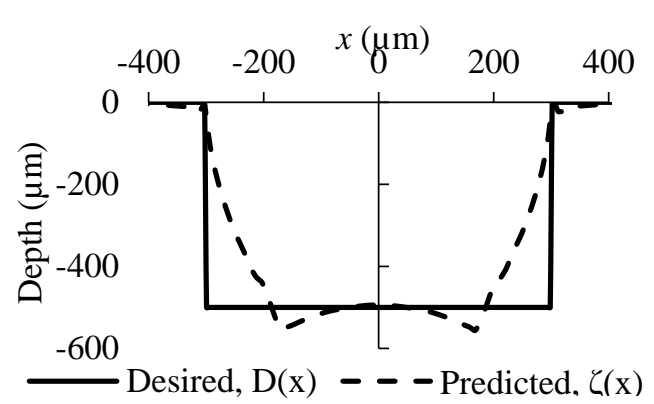

(b)

Figure 3.5.1.1: (a) Predicted scaled erosive efficacy, $E_{r}(x)$, required to produce (b) desired, $D(x)$, rectangular microchannel, with predicted final profile, $\xi(x)$. 


\subsubsection{Trapezoidal}

The approximated superimposed/approximated scaled erosive efficacy, $S(x)$, for the trapezoidal case shown in Figure 3.5.1.2a was produced using the 5 different sources. The machining procedure was repeated $n_{f}=6$ times to machine the measured channel whose profile is shown in Figure 3.5.1.2b. A scanning electron micrograph (SEM) of the machined channel is also shown in Figure 3.5.1.3.

To overcome the accelerated erosion on the flat bottom compared to the sloped sidewalls that is typical of brittle erosion, Figure 3.5.1.2a shows that the $E_{r}$ predicted by the inverse algorithm was higher towards the periphery than at the center. The differences in $E_{r}$ and $S$ reflect the finite width of the source's scaled erosive efficacy $S(x)$ used in approximating $E_{r}$. Nevertheless, Figure 3.5.1.2b shows that the machined trapezoidal cross-section was close to both the desired (5.2\% average absolute difference) and predicted (2.3\% average absolute difference) profiles. Although the channel was nearly flat at the center, a small amount of off-center waviness resulted because of the use of overlapping finite size sources. Nevertheless, the waviness was well predicted and could thus be controlled. Overall, it has been demonstrated that the present inverse modeling technique could be used to produce features with wide bottoms and relatively steep sidewalls, a shape that is contrary to the natural tendency of producing V-shaped channels in the glass when using single straight passes.

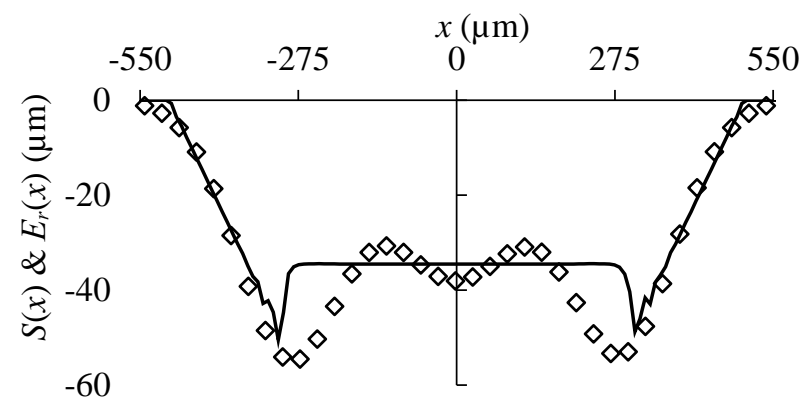

a)

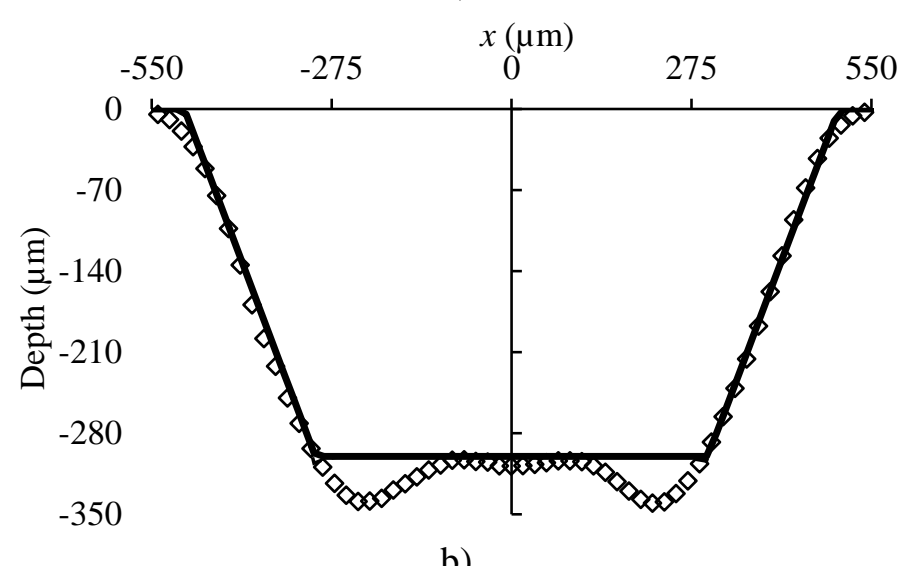

b)

Figure 3.5.1.2: (a) Predicted $E_{r}(x)$ shown with the solid line, and approximate $S(x)$ (shown with the $\diamond$ markers) scaled erosive efficacy required to produce (b) desired, $D(x)$ (solid line), rectangular microchannel, with predicted, $\xi(x)$ (dashed line) and measured final profiles, displayed with $\diamond$ in $(b)$. The predicted surface and the desired surface were so close that they were nearly indistinguishable. 


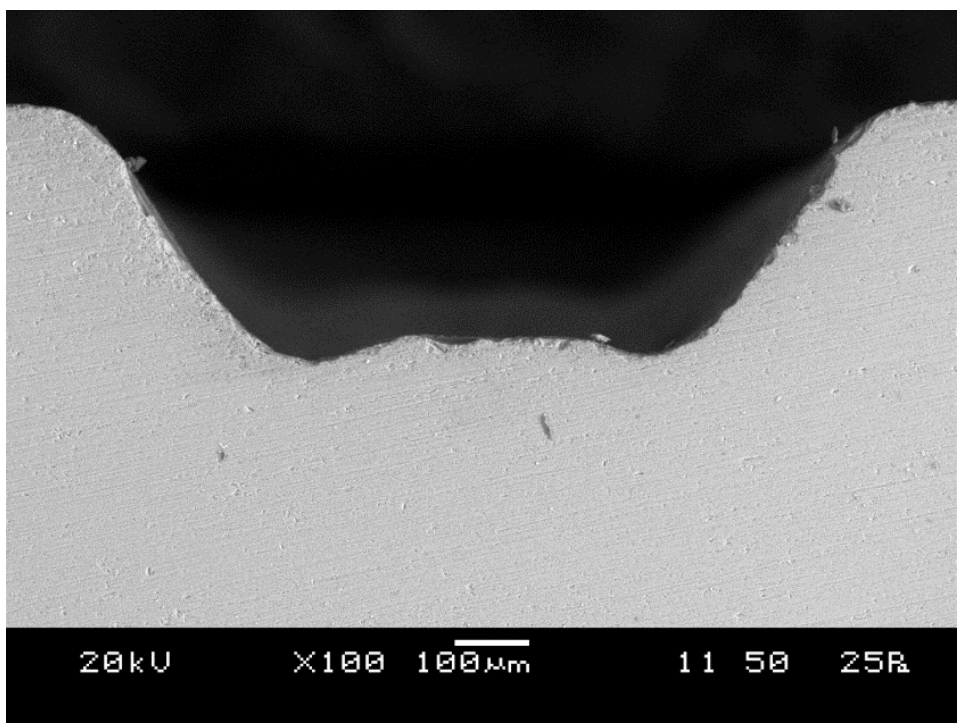

Figure 3.5.1.3: SEM of trapezoidal cross-section machined in the glass.

\subsubsection{Semi-circular}

The approximated superimposed/approximate scaled erosive efficacy, $S(x)$, for the 500, $600,800,700$, and $900 \mu \mathrm{m}$ radius semi-circular micro-channel cross-sections were acquired using the algorithm presented in Section 2.5 and distributed the adjacent passes of the nozzle with the appropriate offsets, scan speeds and the required number of repeats as seen in Table A-1.

In general, the shape of the required scaled erosive efficacies for the circles is characterized by a generally flat, slightly sloped, central portion. This reflects the fact that as the channel becomes deeper, the nature of the brittle erosion (Figure 3.5.1) will tend to accelerate the erosion in the channel center (where particle impacts are near perpendicular) and decrease that on the sloped sidewalls (oblique particle impacts), so that a relatively high scaled erosive efficacy is required off-center to ensure a constant radius of curvature.

The first specimen was a semicircle of $500 \mu \mathrm{m}$ radius with rounded edges. The distribution of the scaled erosive efficacy created by 5 adjacent sources (Figure 3.5.1.4a) may be compared to the required scaled erosive efficacy which was achieved by iterating the algorithm 45 times. The required number of machining repeats was 18 . The average difference between $\xi(x)$ and $D(x)$, i.e. Tol, was $1 \%$. 

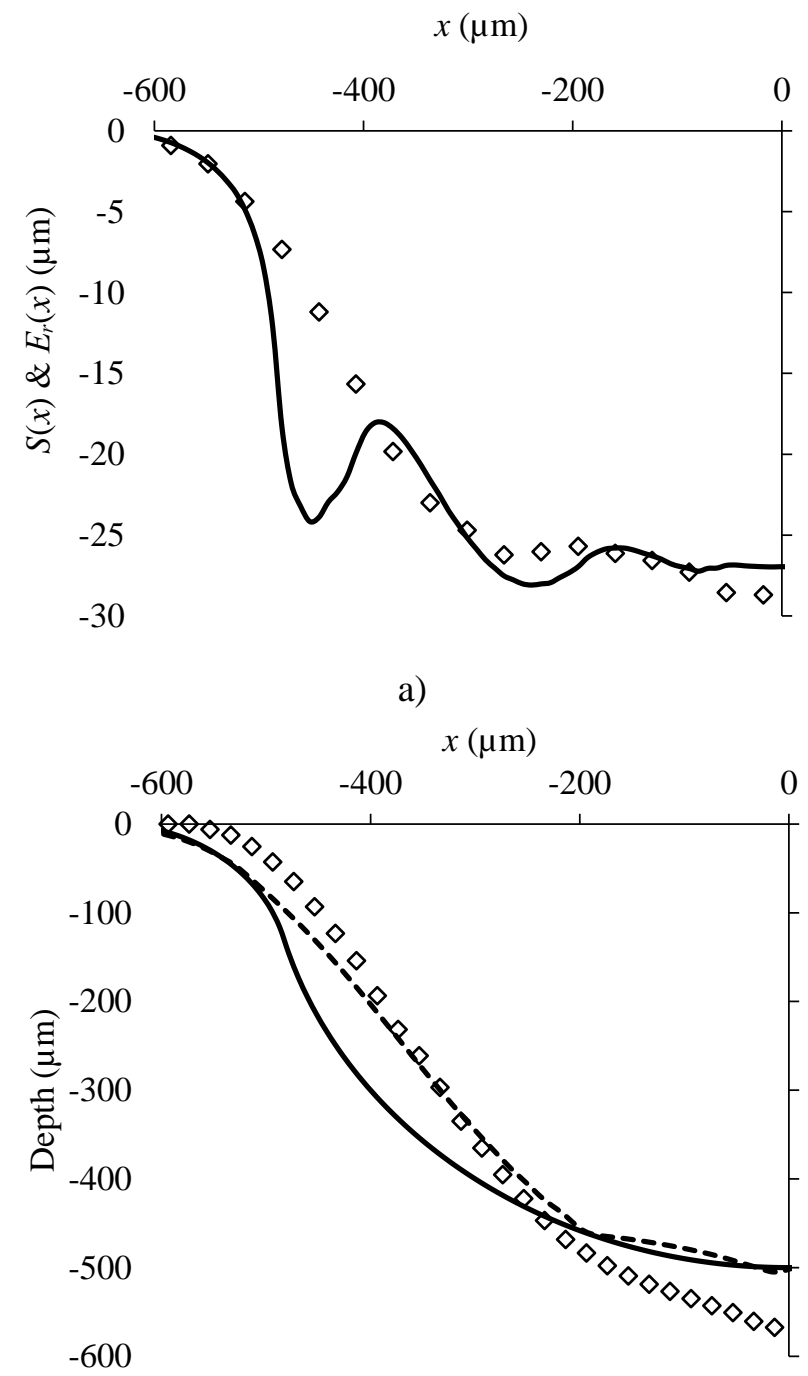

b)

Figure 3.5.1.4: (a) Predicted $E_{r}(x)$ (solid line), and approximate $S(x)(\diamond$ markers) scaled erosive efficacy required to produce (b) desired, $D(x)$ (solid line), $500 \mu \mathrm{m}$ radius semi-circular channel, with predicted, $\xi(x)$ (dashed line) and measured final profiles ( $\diamond$ markers). Five sources were used to create the profile. Only half the symmetric channel is shown.

Figure 3.5.1.4b for the $500 \mu \mathrm{m}$ radius semi-circle shows that the use of the scaled erosive efficacy of only 5 sources, creating $S(x)$, (i.e. 5 adjacent offset passes) resulted in a reasonably good fit of machined (measured) to desired profile (the average difference between the desired and the machined was less than $10.3 \%$ ). The larger deviations in depth in the mid-section of the channel $(51.2 \mu \mathrm{m}$ in average $)$ could be attributed to the number of adjacent sources being limited by the large ratio of $E_{j}(x)$ 's width over that of $D(x)$.

Although an increase in the number of sources would have likely improved the fit, this was not possible since the relative width of the source $(450 \mu \mathrm{m})$ to the desired channel was too large. Therefore, the number of sources was increased for the larger radius circles. As shown in Figure 3.5.1.5b. 
The next semicircle had a radius of $600 \mu \mathrm{m}$ with rounded edges. The required scaled erosive efficacy and the superimposed combination of 9 sources, with scan speeds seen in Table A-1, are presented in Figure 3.5.1.5a after iterating the algorithm 50 times. It was predicted that the scaled erosive efficacy needed to be administered in 20 repeats. The average difference between $\xi(\mathrm{x})$ and $D(\mathrm{x})$ was $1 \%$.
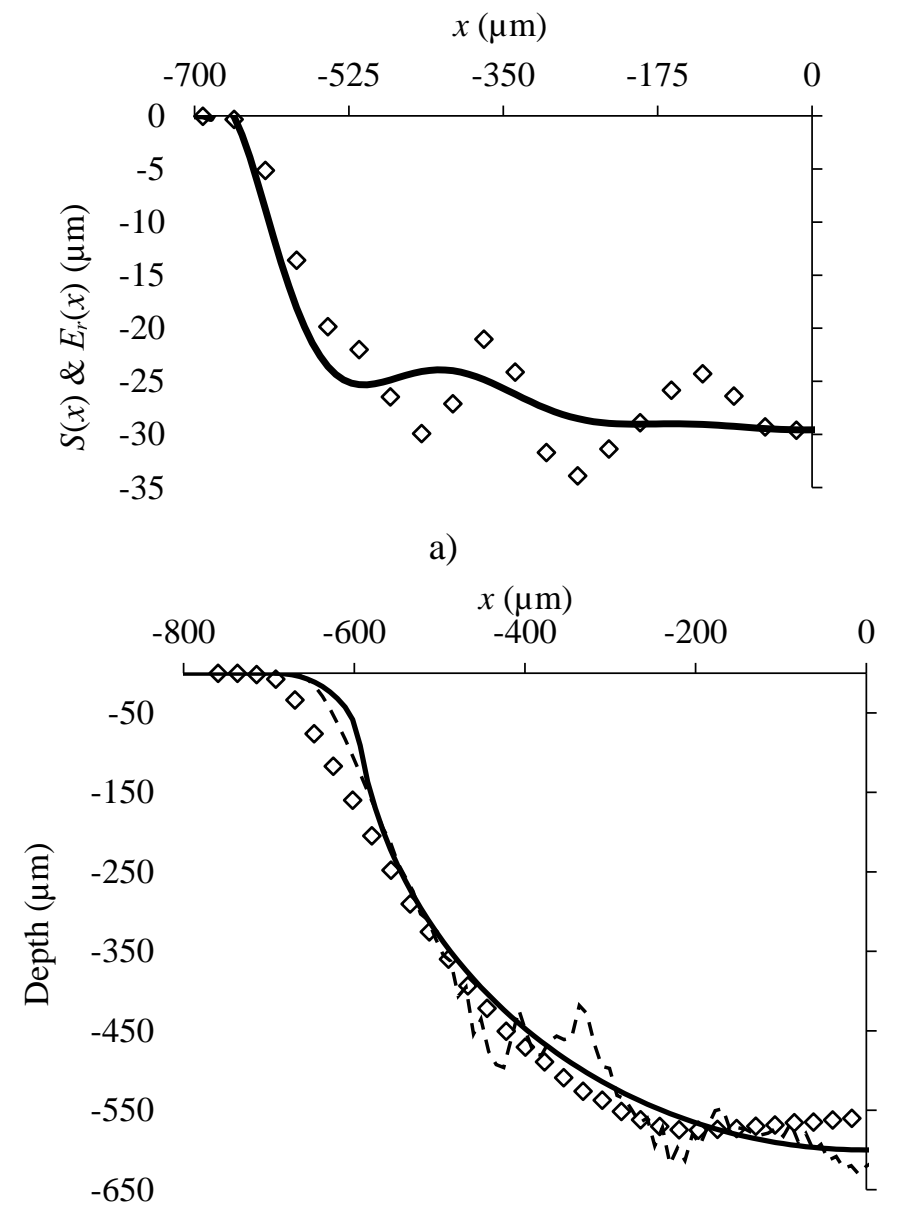

b)

Figure 3.5.1.5: (a) Predicted $E_{r}(x)$ (solid line), and approximate $S(x)$ (dashed line) superimposed scaled erosive efficacy required to produce (b) desired, $D(x)$ (solid line), $600 \mu \mathrm{m}$ radius semi-circular channel, with predicted, $\xi(x)$ (dashed line) and measured final profiles ( $\diamond$ markers). Nine sources were used to create the profile. Only half the symmetric channel is shown.

The profile of the $600 \mu \mathrm{m}$ radius circle machined with 9 sources did indeed significantly improve the fit to the desired over the first $500 \mu \mathrm{m}$ but was approximately half a source width too wide (as evident in Figure 3.5.1.5b). This was because 9 adjacent passes were difficult to fit within the $600 \mu \mathrm{m}$ radius circle. Overall, the average difference in depth between the target and the experiment was below $10.5 \%$.

Even though efforts were made to reduce the effect of the predicted cusps by rounding the desired $D(x)$ (radius of curvature $=100 \mu \mathrm{m}$, as described in Section 2.8) where the semicircle meets the uneroded flat surface, the $E_{r}(x)$ values still exhibit such cusps. Nevertheless, the approximation to the superimposed/approximate scaled erosive efficacy $S(x)$ had the effect of smoothing these cusps (Figure 3.5.1.4a-Figure 3.5.1.8a) so that the resulting machined channels 
(Figure 3.5.1.4b-Figure 3.5.1.8b) were relatively close to those desired. As an example, Figure 3.5.1.9 shows an SEM of the machined channel for the $800 \mu \mathrm{m}$ radius case.

Another successfully machined semicircle is that in Figure 3.5.1.6. The required scaled erosive efficacy and the superposition of adjacent scaled erosive efficacies created by ten sources are presented in Figure 3.5.1.6a. According to the Equation 2-3 for a brittle target, the average difference between $\xi(\mathrm{x})$ and $D(\mathrm{x})$ was $1.1 \%$ (found through 72 iterations of the algorithm), when the sample was eroded under the required scaled erosive efficacy after 24 repeats.

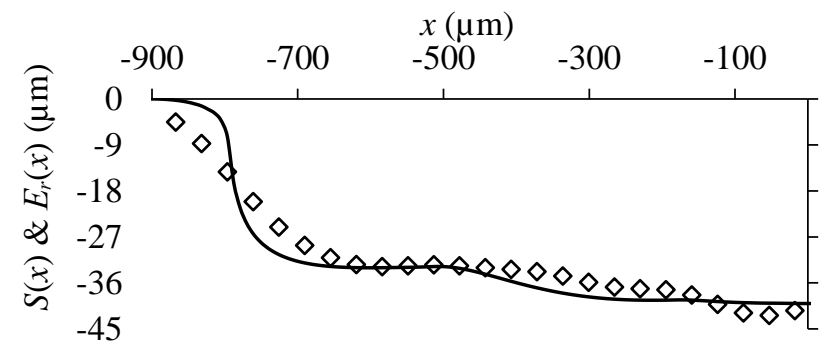

a)

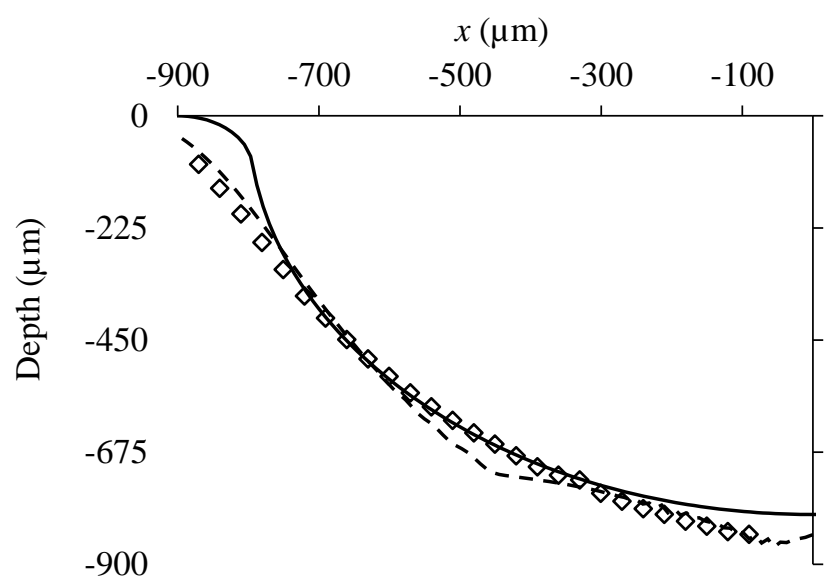

b)

Figure 3.5.1.6: (a) Predicted $E_{r}(x)$ (solid line), and approximate $S(x)(\diamond$ markers) scaled erosive efficacy required to produce (b) desired, $D(x)$ (solid line), $800 \mu \mathrm{m}$ radius semi-circular channel, with predicted, $\xi(x)$ (dashed line) and measured final profiles ( $\diamond$ markers). Ten sources were used to create the profile. Only half the symmetric channel is shown.

Figure 3.5.1.6b demonstrates that increasing the radius of the desired semi-circle to 800 $\mu \mathrm{m}$ resulted in a very good fit between machined and desired (the average difference was less than $5.5 \%$ of the semicircle's depth). In this case, the channel was sufficiently wide to accommodate the ten sources necessary to achieve a good machined resolution.

An additional set of algorithm runs and experiments was undertaken using the number of sources with the highest accurate results (10) adjacent passes to create $700 \mu \mathrm{m}$ and $900 \mu \mathrm{m}$ radius semi-circular cross-sections. The algorithm predicted that the $700 \mu \mathrm{m}$ radius semicircle could be machined after 20 repeats, given the required scaled erosive efficacy seen in Figure 3.5.1.7a, after adjusting the scaled erosive efficacy 55 times. This prediction resulted in only $1 \%$ relative error between $\xi(x)$ and $D(x)$. This scaled erosive efficacy was created using respectively ten sources, $S_{I}(x)$, and eight sources, $S_{2}(x)$. 


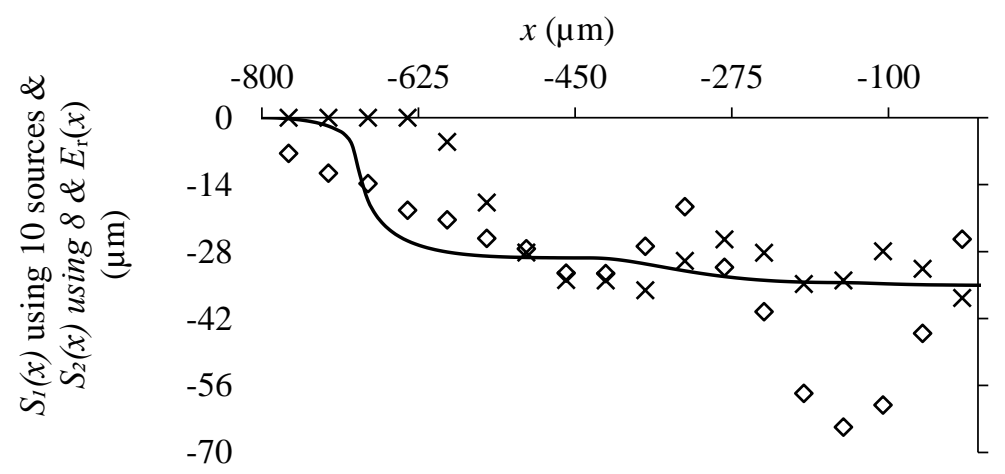

a)

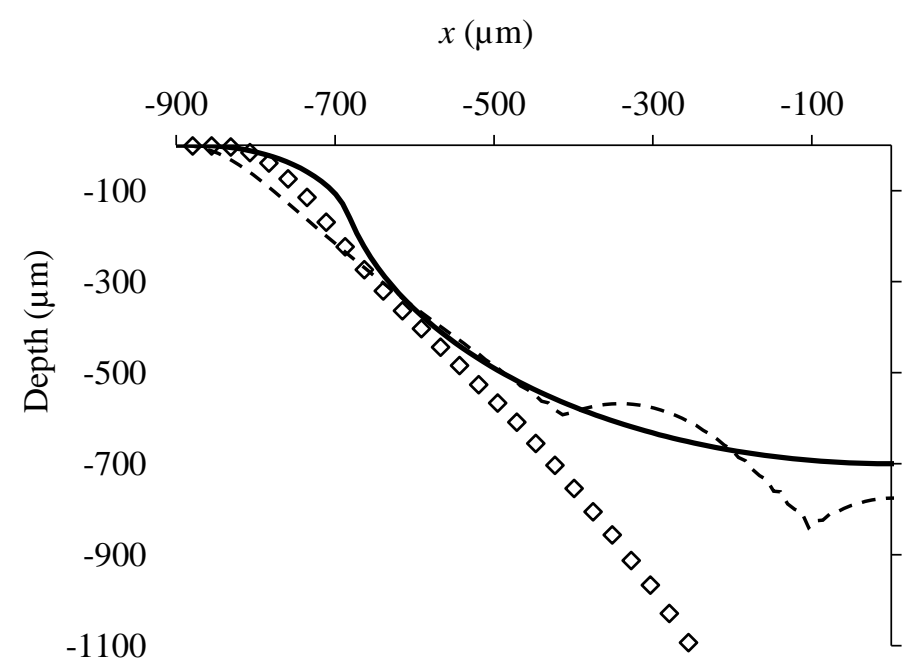

b)

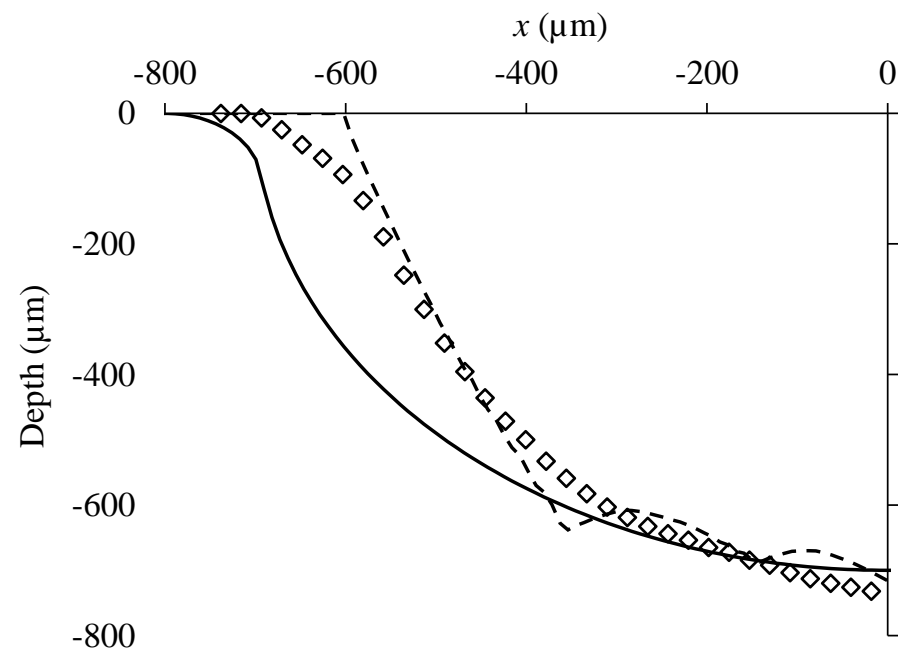

c)

Figure 3.5.1.7: (a) Predicted $E_{r}(x)$ (solid line), and approximate $S_{I}(x)(\diamond$ markers) superimposed scaled erosive efficacy created using 10 sources and $S_{2}(x)$ ( $\times$ markers), using 8 sources required to produce desired, $D(x)$ (solid line), $700 \mu \mathrm{m}$ radius semi-circular channel, with predicted, $\xi(x)$ (dashed line) and measured final profiles ( $\diamond$ markers) using (b) ten and (c) eight sources. Only half the symmetric channels are shown. 
Two results are presented in Figure 3.5.1.7b and Figure 3.5.1.7c using $S_{1}(x)$ and $S_{2}(x)$, respectively. The machined channel seen in Figure 3.5.1.7b was found to dramatically deviate from both the desired and predicted at the center.

However, although the absolute average difference in depth between the desired and measured profiles was $37 \%$, the difference was much smaller at the periphery. For example, it was only $1.6 \%$ between $x=-900 \mu \mathrm{m}$ and $x=-500 \mu \mathrm{m}$ in Figure 3.5.1.7b.

The fact that the predicted and desired final profiles show fairly good agreement points to an issue with the experiment, rather than the modeling. It is likely that this misfit is due to increased particle ricochets from the sidewalls to strike the channel center when a larger number of sources was used. This hypothesis was further strengthened by reducing the number of sources to 8, resulting in the required scaled erosive efficacies of Figure 3.5.1.7c which reduced the average difference in depth for the $700 \mu \mathrm{m}$ radius sample to $9.3 \%$ (Figure 3.5.1.7c). The effect of these particle second-strikes is discussed further in Section 3.6.

Another experiment which emphasized the considerations presented in Section 3.6. was a semicircle of $900 \mu \mathrm{m}$ radius (presented in Figure 3.5.1.8). The required scaled erosive efficacy was predicted by the algorithm after 43 iterations reducing the predicted average difference between $D(x)$ and $\xi(x)$ evolved by $E_{r}(x)$ to $0.73 \%$ if repeated 22 times. 


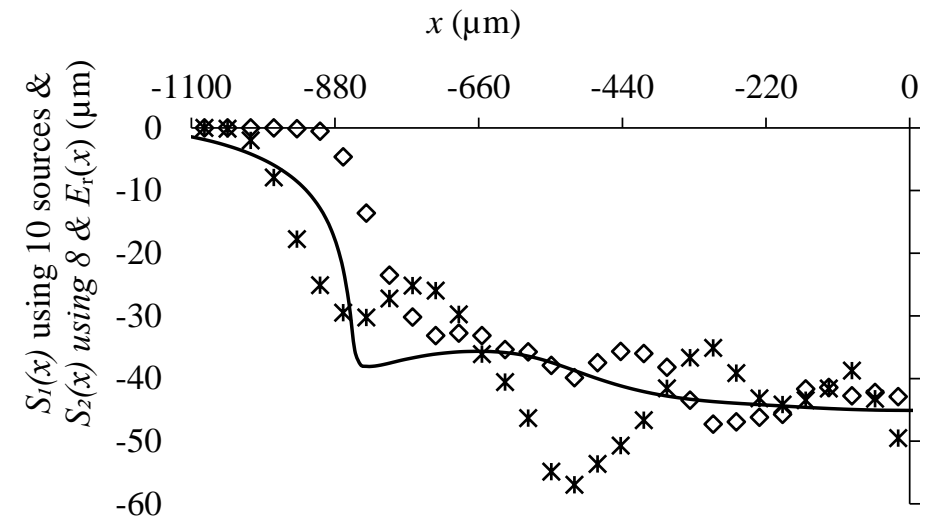

a)

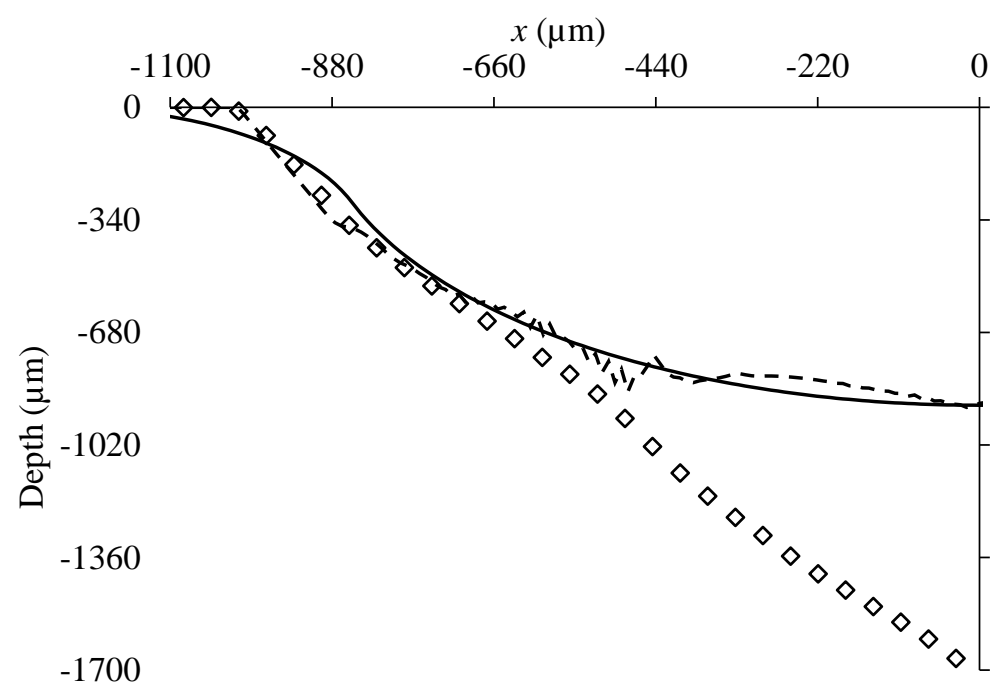

b)

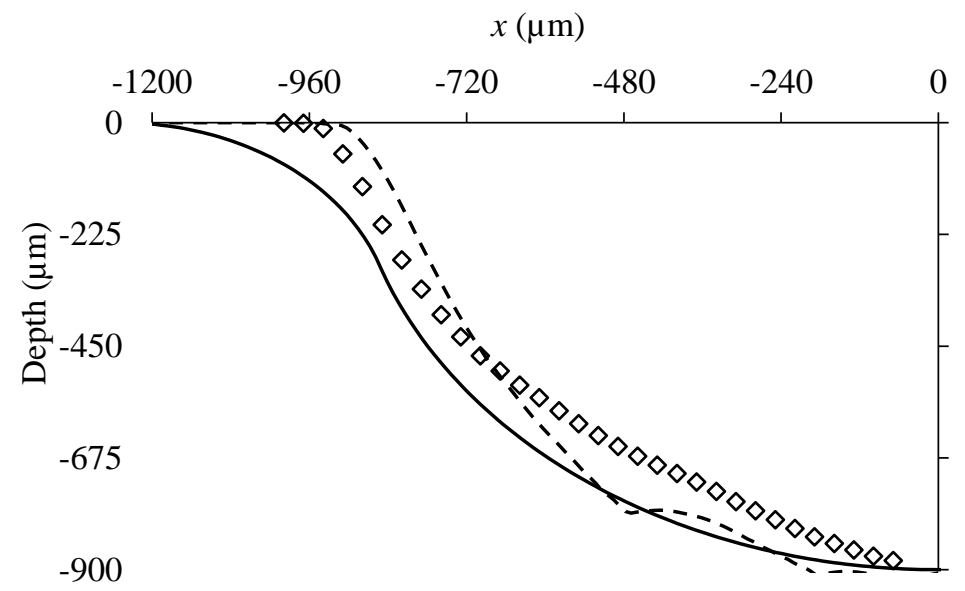

c)

Figure 3.5.1.8: (a) Predicted $E_{r}(x)$ (solid line), and approximate $S_{I}(x)(\diamond$ markers) superimposed scaled erosive efficacy created using 10 sources and $S_{2}(x)$ ( $\times$ markers), using 8 sources required to produce desired, $D(x)$ (solid line), $900 \mu \mathrm{m}$ radius semi-circular channel, with predicted, $\xi(x)$ (solid line) and measured final profiles ( $\diamond$ markers) using (b) 10 and (c) 8 sources. Only half the symmetric channels are shown. 
The results can be compared in Figure 3.5.1.8b and Figure 3.5.1.8c with and without deviations at the center, respectively. The average difference in the experiment presented in Figure 3.5.1.8b was $42 \%$, which was reduced to only $10.9 \%$ after reducing the number of sources.

In summary, despite the tendency for profiles to evolve to have shallow sloped walls in brittle materials, it has been demonstrated that the presented algorithm allows curved surfaces to be machined in such materials. Figure 3.5.1.9 presents the successfully machined $800 \mu \mathrm{m}$ radius semicircle).

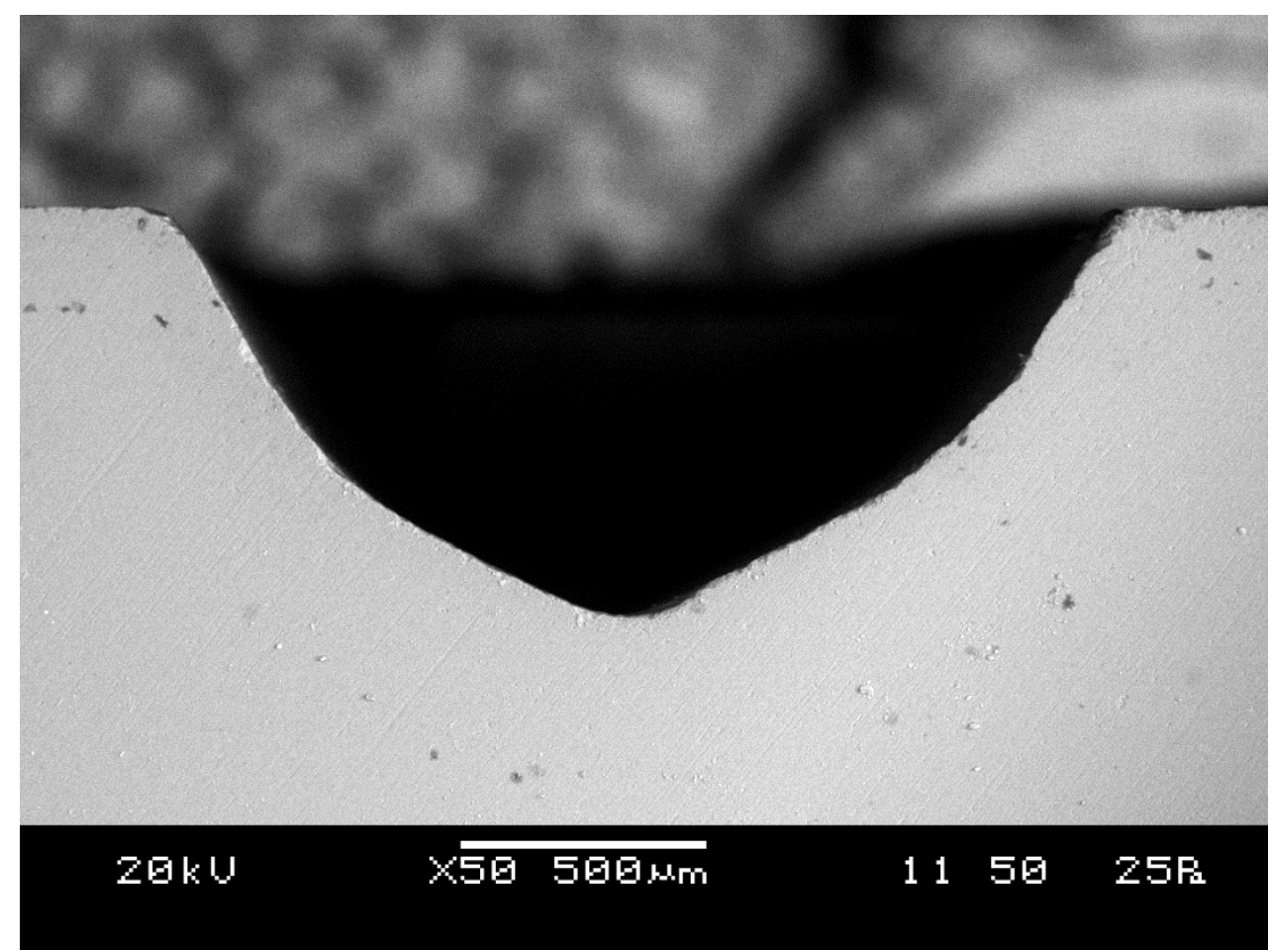

Figure 3.5.1.9: SEM of semicircular cross section channel (radius=800 $\mu \mathrm{m}$ ) machined in the glass the scaled erosive efficacy of which could be described in section 3.3.

\subsubsection{Semicircular Protrusions}

In addition to machining recessed features, it was of interest to determine whether the inverse algorithm could also be used to machine protrusions. As an illustrative example, a semicircular protrusion was chosen as the desired target.

The desired profile shown in Figure 3.5.1.10 was a semicircle of $600 \mu \mathrm{m}$ radius separated from the sidewalls by a flat region $(1400 \mu \mathrm{m}$ wide) with every sharp edge rounded according to the criterion presented in 2.8 The flat region existed only to prevent the sidewalls of the eroded region from restricting the control over the convex topography.

The required scaled erosive efficacy, seen in Figure 3.5.1.10a, was predicted by the algorithm after 100 iterations which reduced the absolute average difference between $\xi(\mathrm{x})$ and $D(\mathrm{x})$ to $0.7 \%$. The predicted $E_{r}(x)$ was simulated using 22 sources with 18 repeats and the scan speeds and offsets seen in Table A-2. 

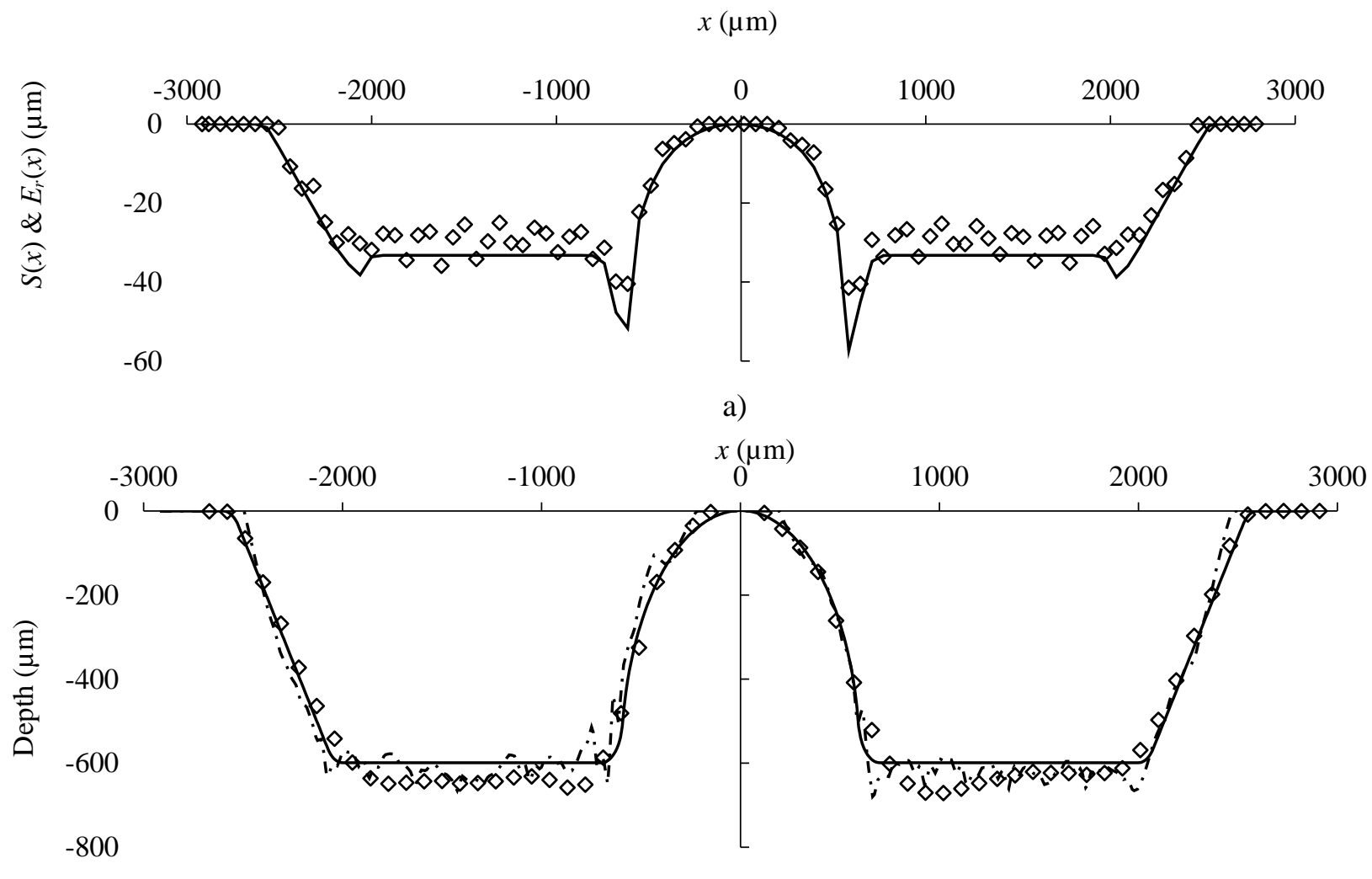

b)

Figure 3.5.1.10: (a) Predicted $E_{r}(x)$ (solid line), and approximate $S(x)$ ( $\diamond$ markers) superimposed scaled erosive efficacy required to produce (b) desired, $D(x)$ (solid line), $600 \mu \mathrm{m}$ radius semi-circular protrusion in the channel, with predicted, $\xi(x)$ (dashed line) and measured final profiles ( $\diamond$ markers). 22 sources were used to create the profile. Only half the symmetric channel is shown.

The experimental, predicted, and desired profiles are shown in Figure 3.5.1.10b. The resulting error between $\xi(x)$ and $D(x)$ was only $4.5 \%$ of the semicircle's radius. The significantly improved accuracy suggested that accuracy in the semi-circular channels of Section 3.5.1.3. was limited by the sidewalls of the channel approaching one other.

Another protruding semicircle, this time with an $800 \mu \mathrm{m}$ radius and $800 \mu \mathrm{m}$ flat region was machined using the inverse method. Figure 3.5.1.11a depicts $S(x)$ and $E_{r}(x)$ which resulted in the average difference between $D(x)$ and $\xi(x)$ evolved by $E_{r}(x)$ to $0.5 \%$ of the semicircle's radius after 100 iterations, using 15 machining repeats. 


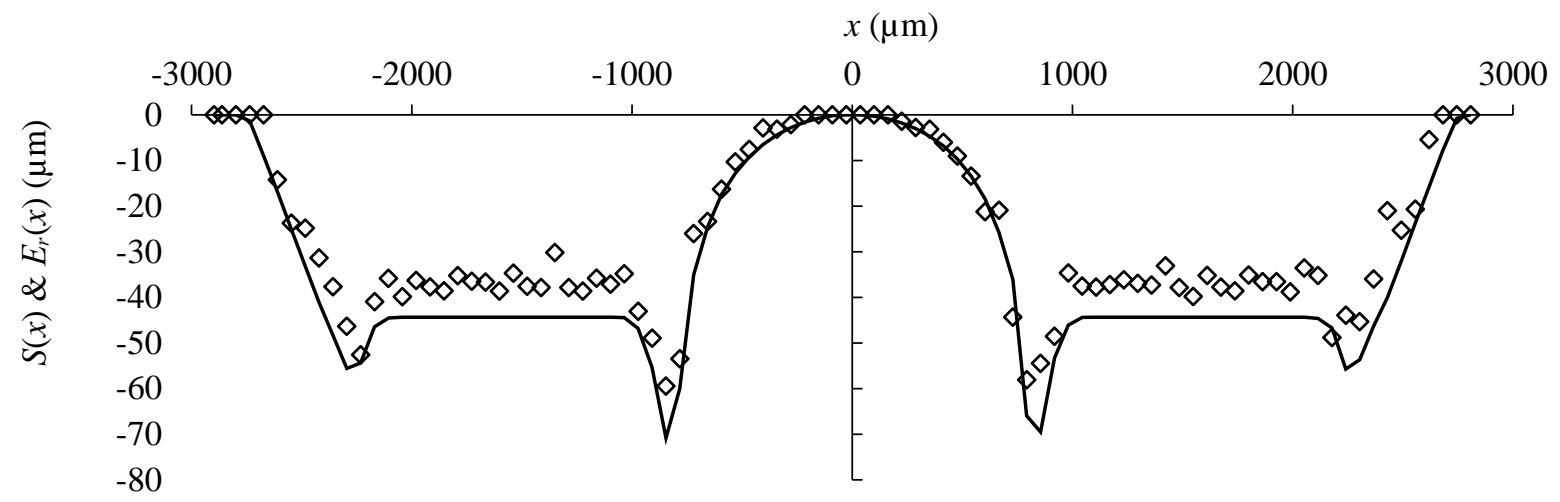

a)

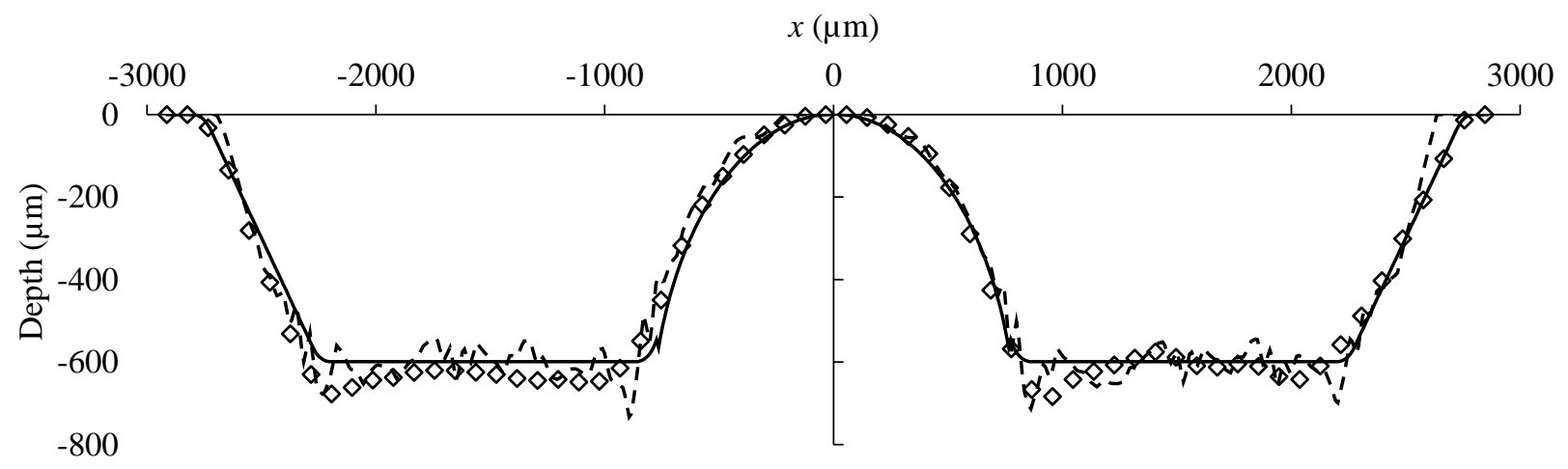

b)

Figure 3.5.1.11: (a) Predicted $E_{r}(x)$ (solid line), and approximate $S(x)(\diamond$ markers) superimposed scaled erosive efficacy required to produce (b) desired, $D(x)$ (solid line), $800 \mu \mathrm{m}$ radius semi-circular protrusion in the channel, with predicted, $\xi(x)$ (dashed line) and measured final profiles ( $\diamond$ markers). 26 sources were used to create the profile. Only half the symmetric channel is shown.

Figure 3.5.1.11b shows the improved accuracy in the machined channel when compared to the measured profile, $D(x)$ or $\xi(x)$ (the average absolute difference in depth was only $3.3 \%$ in the range of $x=-1000 \mu \mathrm{m}$ to $\mathrm{x}=1000 \mu \mathrm{m})$.

Finally, a semicircle protrusion of radius $600 \mu \mathrm{m}$ was machined within a trapezoid of depth $800 \mu \mathrm{m}$ and width $5.6 \mathrm{~mm}$ to ensure that the significant improvement in accuracy of the inverse prediction was not only due to both the center and the edges of the protrusion being in the vicinity of a flat region (over which the depth changed linearly with time). The results can be seen in Figure 3.5.1.12b as eroded under the $E_{r}(x)$ seen in Figure 3.5.1.12a after 18 repeats using 24 sources. 


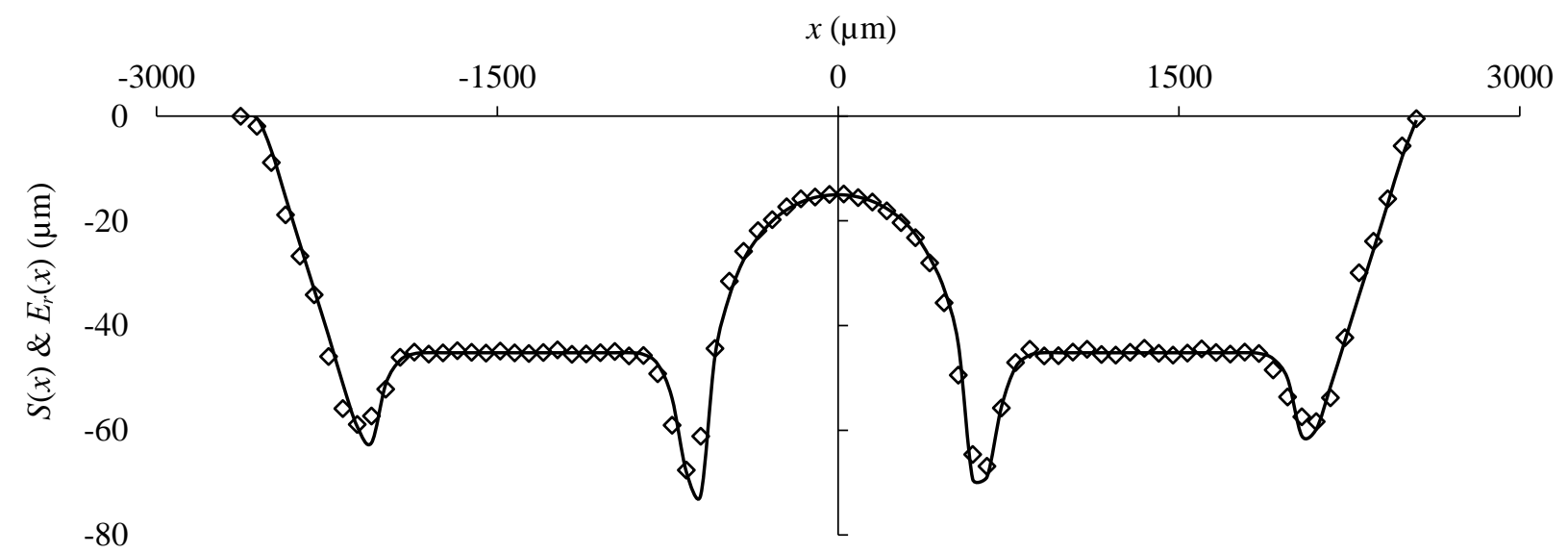

a)

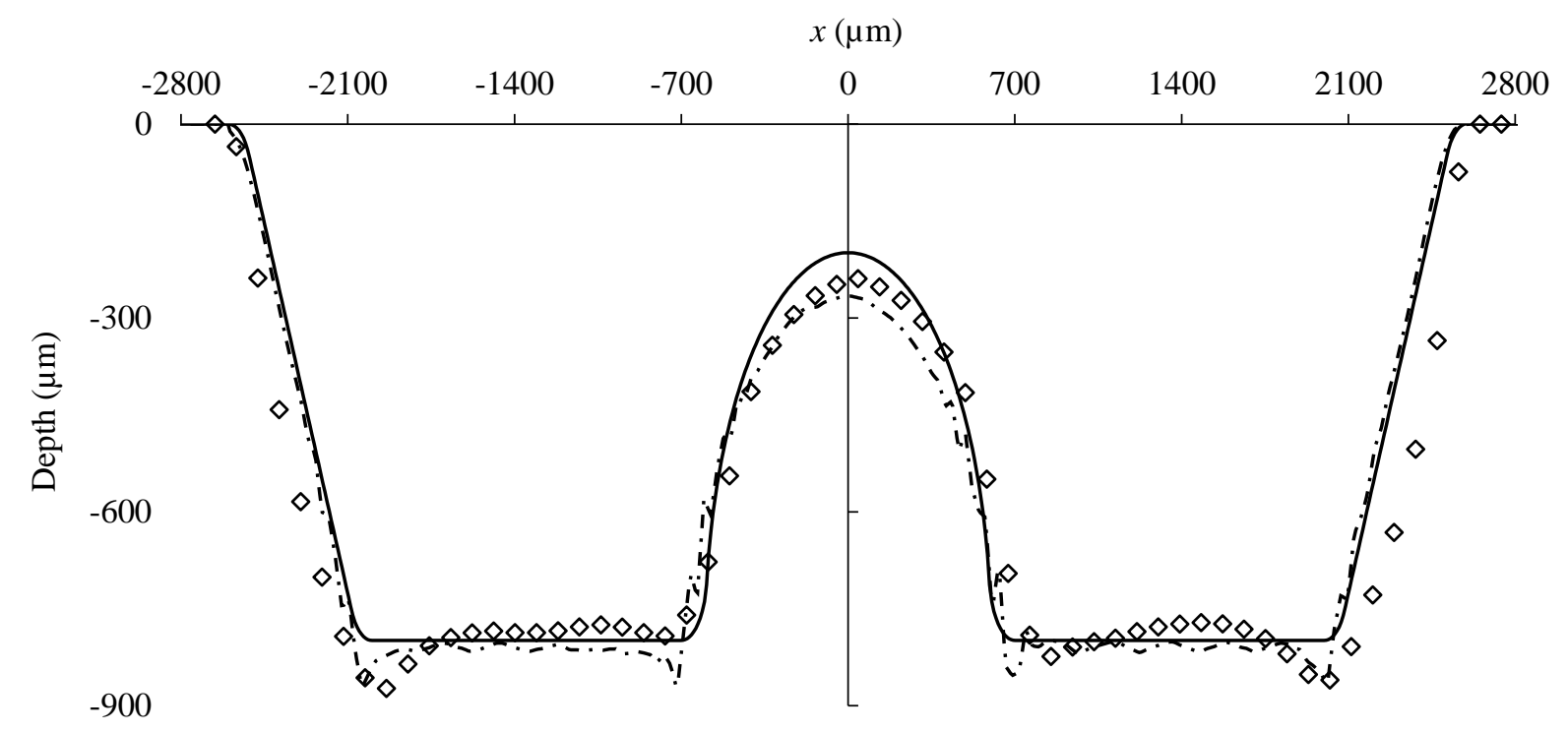

b)

Figure 3.5.1.12: (a) Predicted $E_{r}(x)$ (solid line), and approximate $S(x)(\diamond$ markers) scaled erosive efficacy required to produce $(b)$ desired, $D(x)$ (solid line), $600 \mu \mathrm{m}$ radius semi-circular protrusion in the channel, with predicted, $\xi(x)$ (dashed line) and measured final profiles ( $\diamond$ markers). 22 sources were used to create the profile. Only half the symmetric channel is shown.

The resulting average deviation from $D(x)$ was only $8.2 \%$ of the semicircle's radius.

In summary, the results presented in Figure 3.5.1.10, Figure 3.5.1.11 and Figure 3.5.1.12 provide demonstrated that the inverse method can be used to control the radius of curvature, slope, and depth of machined protruding features. Furthermore, the accurate prediction of the inverse method in creating the final machined profile could be since (compared to Section 3.5.1.3.) the protruded features were located sufficiently far from the sidewalls of the channel. Alternatively, this could be interpreted as an increase in resolution with more sources across the channel, improving the accuracy.

The resolution could be enhanced by using smaller particles or a source with a smaller footprint. Alternatively, in the future, a shadow mask may be integrated into the setup spraying the abrasive to limit the source's width. 


\subsubsection{Channels in PMMA}

\subsubsection{Symmetric Wedges}

The approximated required superimposed/approximate scaled erosive efficacy, $S(x)$, to machine a symmetric wedge in PMMA shown in Figure 3.5.2.1a. It was produced using the 5 adjacent passes with the offsets and scan speeds found in Table A-3 in the appendix. The machining procedure was repeated $n=24$ times to machine the measured channel shown in Figure 3.5.2.1b. The general shape of the scaled erosive efficacy in Figure 3.5.2.1a reflects the ductile erosive nature of PMMA, i.e. a much higher scaled erosive efficacy is required at the center, where particle impacts occur perpendicular to the surface and erosion is relatively low (Figure 3.5.1), than at the periphery, where particles strike at shallower angles, and the erosion is much higher.

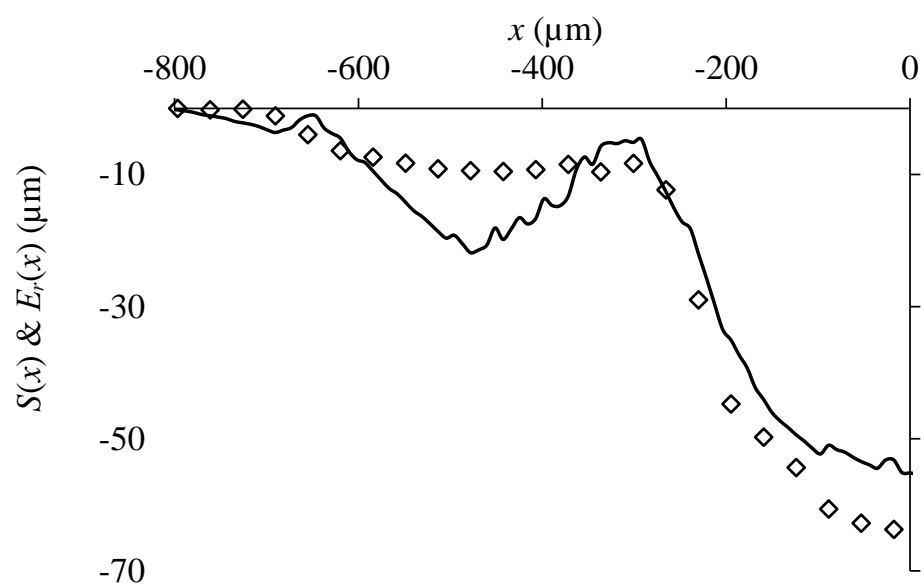

$-70$

a)

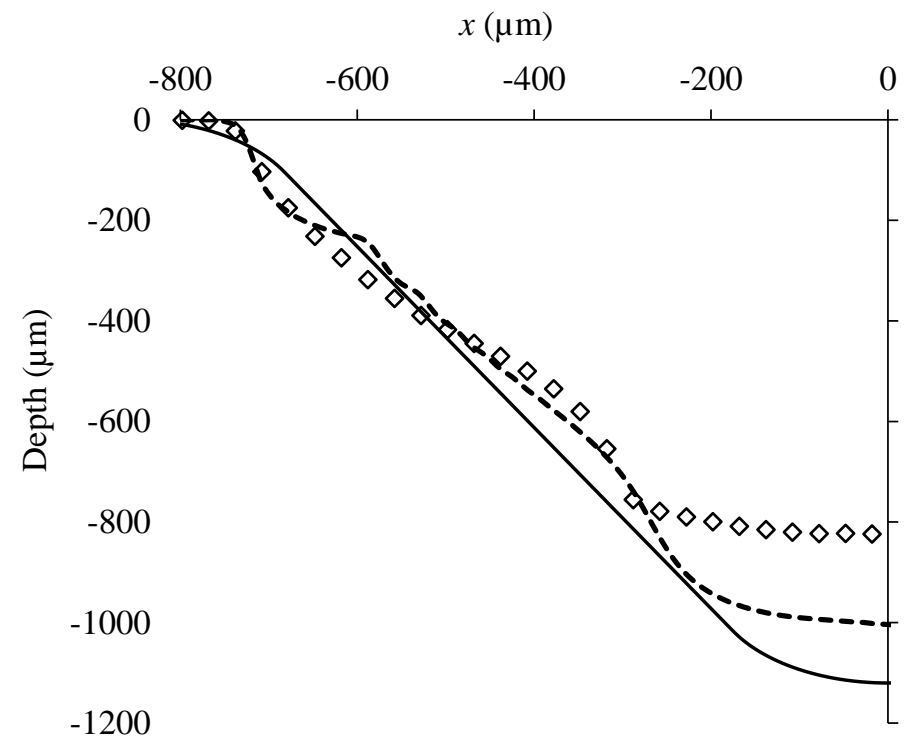

b)

Figure 3.5.2.1: (a) Predicted $E_{r}(x)$ (solid line), and approximate $S(x)(\diamond$ markers) scaled erosive efficacy required to produce (b) desired, $D(x)$ (solid line), symmetric wedge with predicted, $\xi(x)$ (dashed line) and measured final profiles ( $\diamond$ markers). Only half the symmetric channel is shown. 
The average difference between the measured and desired profiles in Figure 3.5.2.1b was $11.2 \%$. The discrepancy is likely mainly due to the fluctuations in the mass flow rate delivered to the wedge's centerline. Since the erosion rate of PMMA is much lower than in the glass [65], a large number (24) of repeats was required, and the level of the abrasive powder in the abrasive hopper significantly reduced, and, as noted in Ref. [77], this significantly decreased the mass flow rate of the abrasive. The cross-section of the final machined channel is shown in Figure 3.5.2.2 below.

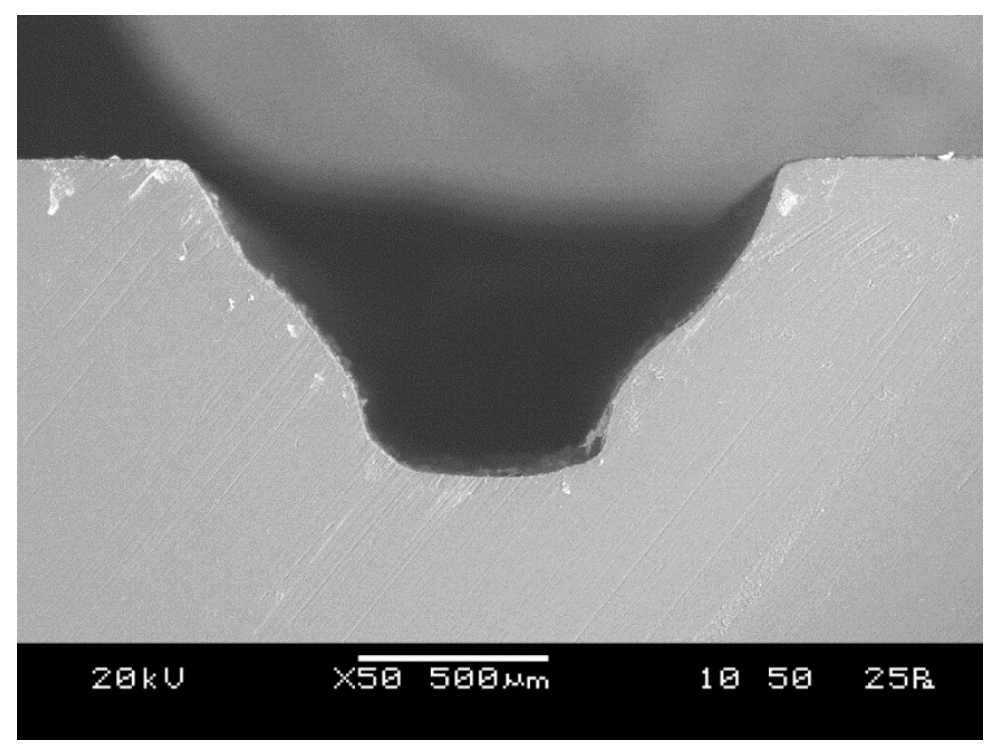

Figure 3.5.2.2: A channel with a wedge-shaped cross-section machined in PMMA

Overall, the relatively good fit of the measured to the desired final profiles demonstrates that the present technique allows sloped symmetric features to be machined in PMMA, despite the natural tendency for very steep walls to form rapidly when machining with one source.

\subsubsection{Asymmetric Wedges}

As mentioned previously, channels machined using a single AJM source in ductile materials such as PMMA evolve to be U-shaped [68] with very steep sidewalls. The slope can be to an extent controlled by superposition of sources with varying scan speeds, however, the steep slope immediately after the edge could not be avoided, since only a single source pass is used at the periphery. In this case, the smallest contribution of the peripheral source to erosion at the periphery was limited by the maximum speed of the positioning stages. Hence, each desired profile hitherto was offset from the un-eroded surface by more than $200 \mu \mathrm{m}$.

An asymmetric wedge with the slope of $20^{\circ}$, which was offset in depth by $230 \mu \mathrm{m}$ and its edges rounded by a minimum radius of $200 \mu \mathrm{m}$, was selected as the desired profile, and the inverse algorithm was used in combination with the Equation 2-4 for a ductile target. $E_{r}(x)$ is presented in Figure 3.5.2.3a, found after 73 iterations resulting in a $0.5 \%$ absolute average difference between $D(x)$ and $\xi(x)$ evolved by $E_{r}(x)$. In addition, the Figure 3.5.2.3a includes the profile made by the superposition of 10 sources (further details are found in Table A-3) which was predicted to erode the surface to the desired shape after 20 repeats. 


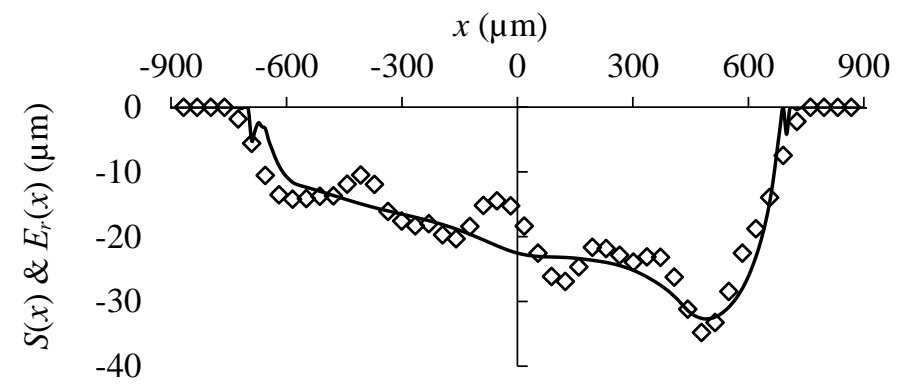

a)

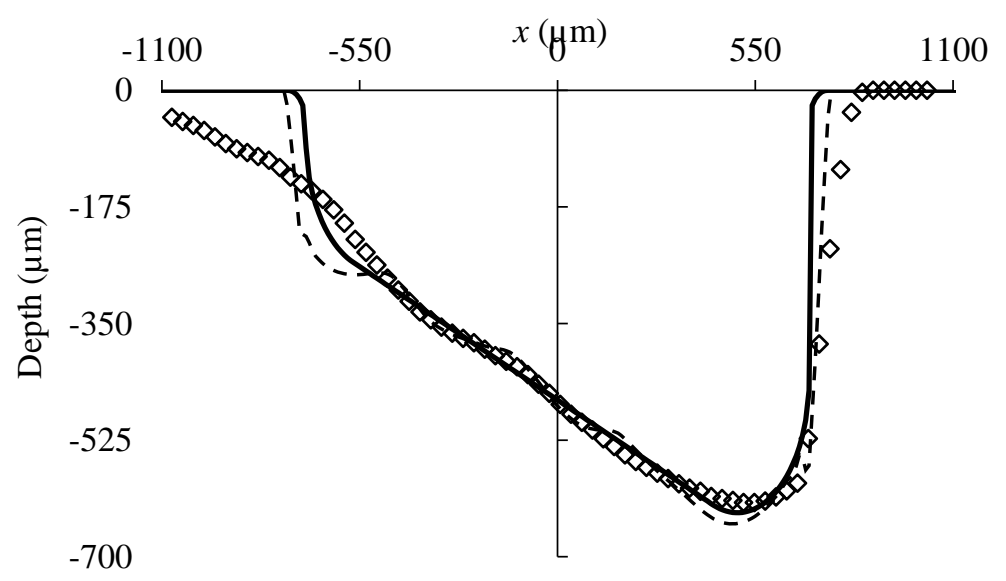

b)

Figure 3.5.2.3: (a) Predicted $E_{r}(x)$ (solid line), and approximate $S(x)(\diamond$ markers) scaled erosive efficacy required to produce (b) desired, $D(x)$ (solid line), $20^{\circ}$ asymmetric wedges with predicted, $\xi(x)$ (dashed line) and measured final profiles $(\diamond$ markers).

The result of erosion under the specified scaled erosive efficacy is displayed in Figure 3.5.2.3b with the predicted and desired target. The average deviation from depth across the channel was $6.3 \%$ of the target's depth (measured from $x=-450 \mu \mathrm{m}$ to $x=1000 \mu \mathrm{m}$ ) proving relative accuracy.

Despite the general shape of the scaled erosive efficacy itself approaching an asymmetric wedge, of an extremely small slope, the proximity of the right sidewall featured a 'bulge' (with a higher radius of curvature compared to the edges). This was reflective of the fact that the slope must very slowly develop in order to ensure it counteracts the natural tendency to rapidly increase in ductile materials where erosion is maximum at oblique particle incidence. The rounded 'bulge' in the scaled erosive efficacy towards the right-hand periphery reflects the much larger scaled erosive efficacy required where the desired profile needed to be flatter, given the much lower erosion rate of flat than sloped surfaces that occurs in ductile materials.

The next asymmetric desired wedge had a slope of $30^{\circ}$ with rounded edges, $250 \mu \mathrm{m}$ initial depth offset and a 'bulge' radius of $400 \mu \mathrm{m}$ (this radius may be increased so that the right edge would form a semi-flat area as wide as a single nozzle's scaled erosive efficacy in PMMA). $S(x)$, created using ten sources, and $E_{r}(x)$ are seen in Figure 3.5.2.4a. With 10.3\% deviation between $D(x)$ and $\xi(x)$ evolved by $E_{r}(x), E_{r}(x)$ was derived after 40 iterations, requiring 20 repeats. 


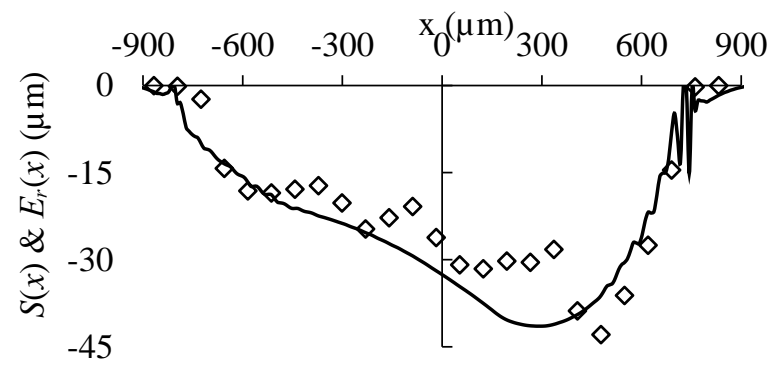

a)

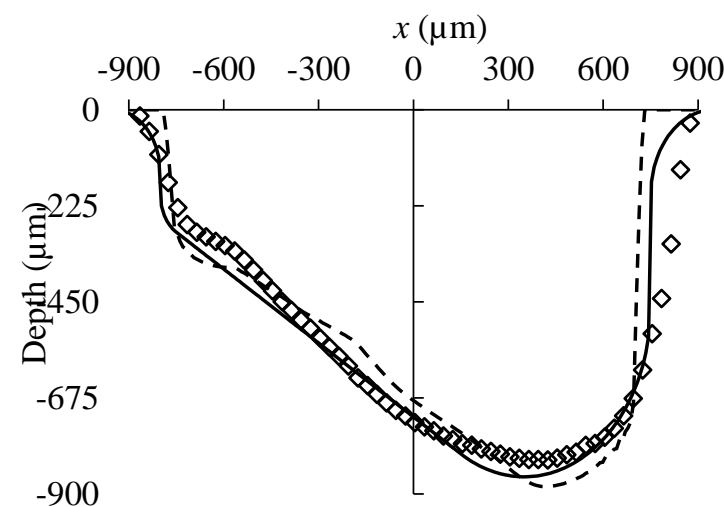

b)

Figure 3.5.2.4: (a) Predicted $E_{r}(x)$ (solid line), and approximate $S(x)$ ( $\diamond$ markers) scaled erosive efficacy required to produce (b) desired, $D(x)$ (solid line), $20^{\circ}$ asymmetric wedges with predicted, $\xi(x)$ (dashed line) and measured final profiles $(\diamond$ markers).

The measured profile, shown in Figure 3.5.2.5b, demonstrated agreement with both the predicted and the desired profile (the average error between the measured profile and the target was only $8.3 \%$ of the target's maximum depth). Figure 3.5.2.5 shows an SEM picture of the machined channel's cross-section.

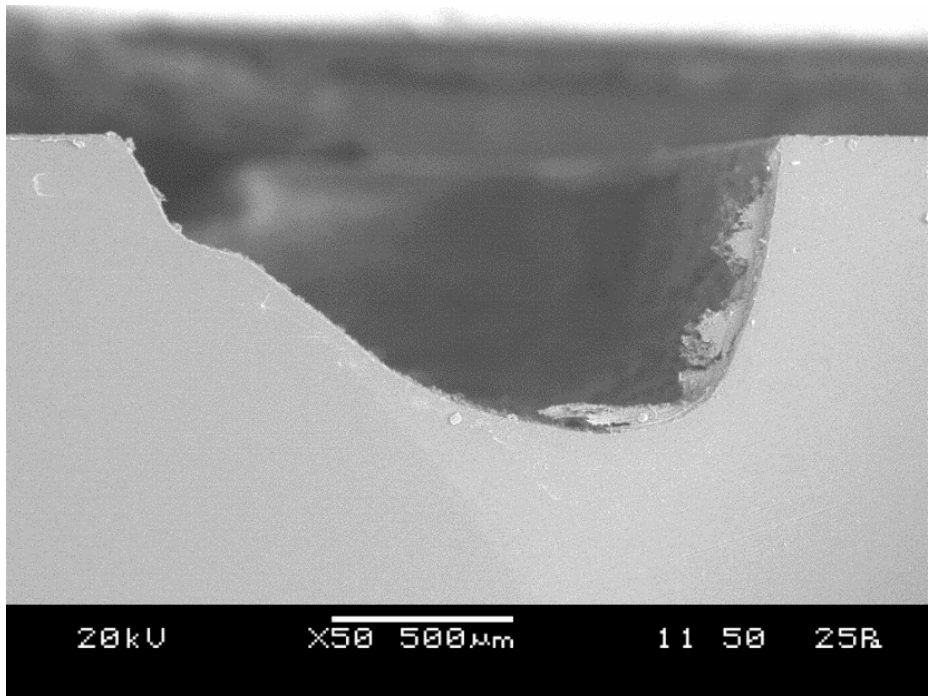

Figure 3.5.2.5: The asymmetric wedges cross-section with a $30^{\circ}$ incline machined in PMMA. Note, the articles at the right side of the channel were created in the cutting process as burs and were not the result of AJM machining. 
Finally, the asymmetric wedge seen in Figure 3.5.2.6 was machined, having an incline of $45^{\circ}$ with rounded corners, a depth offset of $120 \mu \mathrm{m}$ and a radius of curvature equal to $400 \mu \mathrm{m}$ to describe the flat region close to the right sidewall. Figure 3.5.2.6a includes $E_{r}(x)$, created by applying the algorithm through 100 iterations, and $S(x)$ which was the superimposed/approximate scaled erosive efficacy of 9 sources as listed in Table A-3.

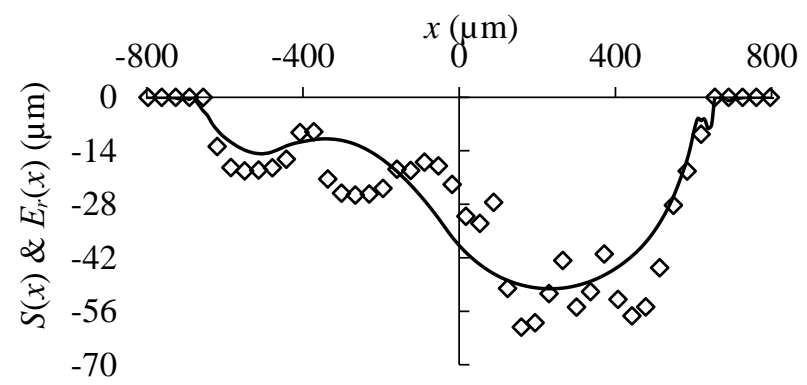

a)

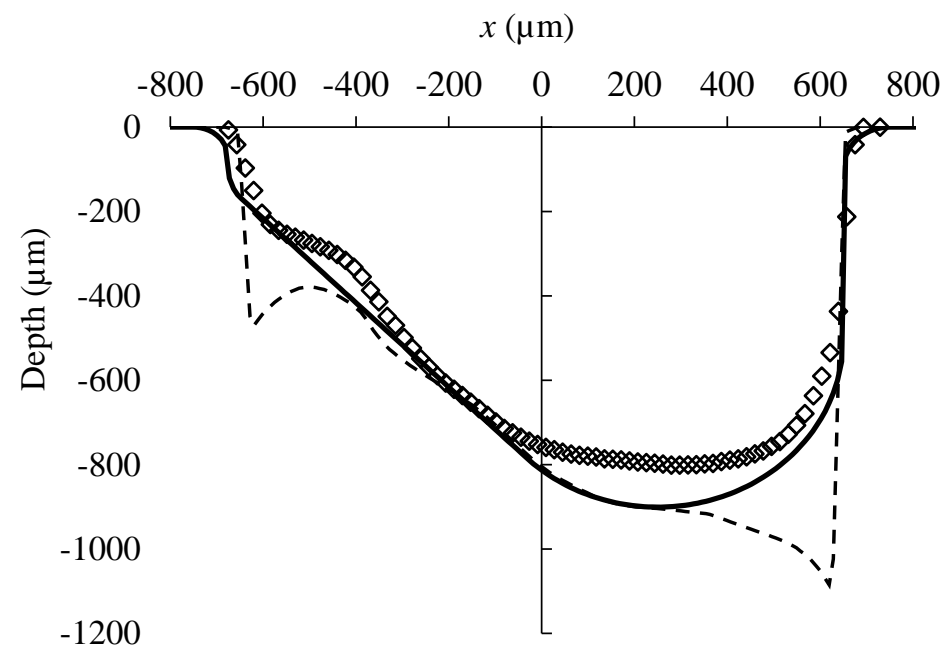

b)

Figure 3.5.2.6: (a) Predicted $E_{r}(x)$ (solid line), and approximate $S(x)(\diamond$ markers) scaled erosive efficacy required to produce (b) desired, $D(x)$ (solid line), $45^{\circ}$ asymmetric wedges with predicted, $\xi(x)$ (dashed line) and measured final profiles $(\diamond$ markers).

The profile in Figure 3.5.2.6b which only deviated by $6.2 \%$ average in depth from the desired profile, was machined. At the points of maximum depth, the overestimation of depth and formation of sharp cusps in the predictions were as a result of limitations of the surface evolution model and not the algorithm. Hence, a slight change from the scaled erosive efficacy to the superposition of the sources, from $E_{r}(x)$ to $S(x)$, resulted in presence of sharp cusps in $\xi(x)$ and overestimation of depth, which could be seen in Figure 3.5.2.6, in the proximity of each sidewall (in practice, the trenches near the sidewalls were not machined using the same $S(x)$ ). 


\subsection{Second-Strike Limitation}

As mentioned in Section 3.5.1.3, particle second-strikes can deleteriously affect the predictions of the algorithm. It was therefore of interest to determine under which condition second-strike would be probable and how it would contribute to erosion. Thus, a ray-tracing analysis was performed to determine the locations of second-strikes. Although this phenomenon was studied, unfortunately, the calculations did not yield a general all-encompassing criterion.

The range over which the particles would strike a second time was found between each repeat. This was done by tracing every particle, assumed to be incident vertically from a source immediately above a lateral location $x_{0}$. Using the classical rigid body dynamics analysis of Brach [79], the angle of incidence of this particle with the evolved surface predicted by Equation 2-3 was found. Then the coefficient of restitution and the coefficient of Coulomb friction $(0.5$ and the range 0.5-0.7, respectively, from Slikkerveer and in't Veld [23]), were used to find the rebound angle and velocity (appendix A.3). Using this information, the location of the second-strike of the rebounding particle was determined (assuming interaction of the particle with the incoming air was negligible) by ray-tracing. $S(x)$ was then modified, using the ratio of the particle's speed after the first impact over that of prior to the impact, to consider the oblique erosion at the secondstrike's location and projected along the vertical to find the contribution of the second-strike to the material removal per repeat.

This formed the algorithm seen in the Figure A.2. This consideration was made for the location of the surface at every repeat up to $n_{f}$ and a range of second-strikes was calculated. When applied to the $900 \mu \mathrm{m}$ and the $700 \mu \mathrm{m}$ semicircles, it was observed that the range over which second-strike occurred was reduced by $72.2 \%$ if $S_{2}(x)$ was used instead of $S_{1}(x)$.

\subsection{Summary}

Chapter 2 employed the inverse algorithm which predicted the required erosive footprint in chapter 2 to machine features ranging from a trapezoid of $300 \mu \mathrm{m}$ depth, semicircles of radii $500 \mu \mathrm{m}-900 \mu \mathrm{m}$, semicircular protrusions with $600 \mu \mathrm{m}$ and $800 \mu \mathrm{m}$ radius with the former being machined $200 \mu \mathrm{m}$ below the free surface of the brittle target (borosilicate glass). Overall, the accuracy of the machined features was such that the maximum average deviation from depth was only $10.9 \%$, proving the algorithm's application as an inverse method for AJM using adjacent passes. Also, the ductile target (PMMA) was machined to create a symmetric ramp and three different asymmetric wedges of inclines $20^{\circ}, 30^{\circ}$ and $45^{\circ}$ with the maximum average deviation in depth measured to be as low as $8.3 \%$. 


\section{Conclusion and Future Work}

\subsection{Conclusion}

This thesis introduced and experimentally verified an inverse method to predict the required erosive efficacy to machine desired topographies of high A.R. in both brittle and ductile targets. The predicted profiles $\xi(x, n)$ closely matched the desired targets with only less than $8 \%$ average difference in depth predicted for the glass targets and $0.4 \%$ difference for the PMMA.

The major conclusions can be summarized as:

- The algorithm's output accurately predicted the $E_{r}(x)$ for desired profiles of aspect ratios close to 0.5 (previously reported to be the limit of surface evolution application). It allowed machining round profiles in brittle materials, and sloped ones, even asymmetric targets, in ductile materials, contrary to the natural tendency of surface evolution in those materials.

- Features located farther from the edges of the channel could be machined with higher accuracy.

- The decrease of the source's width relative to that of the desired profile improved the resolution since the optimization process could more flexibly distribute the scaled erosive efficacies.

- The increment in the number of sources optimized and used to machine the surface was limited by the second-strike effect which resulted in secondary erosion, not predicted by the surface evolution method.

- In a limited fashion, a guideline was provided in section 3.6, which predicted the lateral range over which the sources could be distributed that consequently suggested fewer sources to be applied to machine the desired feature.

- The algorithm performed better for the ductile (PMMA) features compared to the brittle (glass) ones because the ductile ones naturally developed steeper sidewalls. The higher erosion near the profile edges for ductile materials meant that the sharp edges could be more easily defined in that case.

- The desired profile requiring a higher number of repeats (the $45^{\circ}$ asymmetric wedge) deviated the most from the model's prediction where it was deepest, due to a drop in the average mass flow rate after multiple repeats. 


\subsection{Future Work}

Despite its accuracy and proven flexibility, the inverse could improve with minor adjustments in the future:

* It seems plausible that the algorithm's accuracy would improve if a modified surface evolution model was used which predicted the erosion due to second-strike. The algorithm seems able to counter the accumulation of second-strike causing the material's eroded surface to be much deeper than $D(x)$ since the section 2.5's procedure of adjusting $E_{r}(x)$ has proven capable of reducing the scaled erosive efficacy where an ill-suited initial guess caused a similar deviation.

* The algorithm adjusts the required machining parameters independent of the surface evolution model, suggesting that it could be employed to machine features in processes similar to AJM, namely AWJM and EDM.

* The resolution could be enhanced by using smaller particles or a source with a smaller footprint. Alternatively, a shadow mask may be integrated into the setup spraying the abrasive to limit the source's width.

* Hypothetically, this method could be used to machine 3D features by sectioning the feature in multiple parallel planes, applying the inverse algorithm to each individual profile and finding the optimal path for a pre-determined number of sources creating each profile's $S(x)$.

* If optimization was to be applied directly to $S(x)$, the predictions of the required scaled erosive efficacy would benefit from creating an automated condition for the viscous smoothness factor (see section 2.3) for each iteration's $S(x)$ such that cusps would not appear (see section 2.13.1).

* The roughness of some of the machined channels could be improved by post-processing methods such as post blasting, use of water jet passes or, in case of glass, using HF wet etching.

\subsection{Contributions}

The main novel contributions of this thesis were:

> Introducing a novel algorithm that uses the semi-analytical surface evolution model, for both ductile and brittle targets, to predict the required distribution of the scaled erosive efficacy to machine high A.R. channels and protrusions.

$>$ Implementation of the GRG-nonlinear optimization model in combination with the surface evolution to predict the required position and strength of the sources required to machine a desired high A.R. feature using adjacent pass AJM.

$>$ Verifying the algorithm for several unmachined cross-sectional channel shapes. 


\section{Appendix:}

\section{A.1. Table of Machining Parameters for Channels in Glass}

Table A-1 includes the location of the abrasive sources, their scan speed, the mass flow rate and the number of iterations to determine their $E_{r}(x)$ using the inverse method (introduced in chapter 2) for eroding the semicircular profiles of Section 3.5.1.3. in borosilicate glass using the experimental setup described in section.

Table A-1: The machining parameters for creating the semicircular features in the brittle glass

\begin{tabular}{|c|c|c|c|c|c|c|c|c|c|c|c|}
\hline Feature & Parameter & \multicolumn{10}{|c|}{ Source } \\
\hline \multirow{2}{*}{ 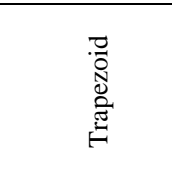 } & Offsets $(\mu \mathrm{m})$ & 0 & 160 & 290 & 417 & 581 & & & & & \\
\hline & Number of repeats & \multicolumn{10}{|c|}{6} \\
\hline \multirow{2}{*}{$\begin{array}{l}\Xi \\
\frac{3}{8} \\
8 \\
\frac{0}{0}\end{array}$} & Scan speed $\left(v_{s}\right)_{K}(\mathrm{~mm} / \mathrm{s})$ & 5 & 4.545 & 2.564 & 4.545 & 5 & - & - & - & - & - \\
\hline & Offsets $(\mu \mathrm{m})$ & 0 & 100 & 330 & 560 & 660 & - & - & - & - & - \\
\hline \multirow{4}{*}{$\frac{\Xi}{8} \frac{0}{0} \frac{0}{0}$} & Scan speed $\left(v_{s}\right)_{K}(\mathrm{~mm} / \mathrm{s})$ & 5.28 & 8.181 & 7.688 & 8.125 & 3.816 & 8.125 & 7.688 & 8.181 & 5.28 & - \\
\hline & Offsets $(\mu \mathrm{m})$ & 0 & 132 & 262 & 293 & 492 & 692 & 722 & 852 & 985 & - \\
\hline & Mass flow rate (g/min) & \multicolumn{10}{|c|}{$\begin{array}{r}1 \\
5.4\end{array}$} \\
\hline & Number of repeats & \multicolumn{10}{|c|}{22} \\
\hline \multirow{2}{*}{$\frac{\Xi}{8} \frac{0}{\mathscr{C}}$} & Scan speed $\left(v_{s}\right)_{K}(\mathrm{~mm} / \mathrm{s})$ & 5.822 & 8.333 & 6.250 & 5.263 & 5.263 & 10 & 6.667 & 9.901 & 5.263 & 5.556 \\
\hline & Offsets $(\mu \mathrm{m})$ & 0 & 150 & 300 & 475 & 650 & 750 & 900 & 990 & 1170 & 1385 \\
\hline \multirow{2}{*}{ 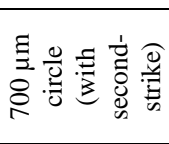 } & Mass flow rate $(\mathrm{g} / \mathrm{min})$ & \multicolumn{10}{|c|}{14.5} \\
\hline & Number of repeats & \multicolumn{10}{|c|}{20} \\
\hline \multirow{4}{*}{ 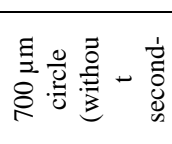 } & Scan speed $f_{i}(\mathbf{m m} / \mathbf{s})$ & 11.429 & 17.778 & 59.259 & 15.595 & 15.595 & 59.259 & 17.778 & 11.429 & - & - \\
\hline & Offsets $(\mu \mathrm{m})$ & 0 & 190 & 250 & 375 & 525 & 650 & 710 & 900 & - & - \\
\hline & Mass flow rate (g/min) & \multicolumn{10}{|c|}{16} \\
\hline & Number of repeats & \multicolumn{10}{|c|}{20} \\
\hline \multirow{4}{*}{ 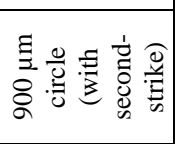 } & Scan speed $\mathbf{f}_{\mathrm{i}}(\mathrm{mm} / \mathrm{s})$ & 11.429 & 8.889 & 6.914 & 8 & 12.5 & 12.5 & 8 & 6.914 & 8.889 & 11.429 \\
\hline & Offsets $(\mu \mathrm{m})$ & 0 & 230 & 400 & 625 & 810 & 890 & 1075 & 1300 & 1470 & 1700 \\
\hline & Mass flow rate $(\mathrm{g} / \mathrm{min})$ & \multicolumn{10}{|c|}{14.5} \\
\hline & Number of repeats & \multicolumn{10}{|c|}{22} \\
\hline \multirow{3}{*}{ 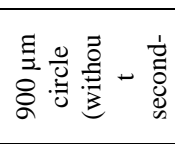 } & Scan speed $\mathrm{f}_{\mathrm{i}}(\mathrm{mm} / \mathrm{s})$ & 9.524 & 8.889 & 6.667 & 14.815 & 14.815 & 6.667 & 8.889 & 9.524 & - & - \\
\hline & Offsets $(\mu \mathrm{m})$ & 0 & 240 & 490 & 720 & 800 & 1030 & 1280 & 1520 & - & - \\
\hline & Mass flow rate (g/min) & \multicolumn{10}{|c|}{$\begin{array}{c}1 \\
17.9 \\
\end{array}$} \\
\hline
\end{tabular}

Table A-2 includes the parameters used to machine the semicircular protrusions in the glass, as well as the number of iterations in the inverse algorithm to define $E_{r}(x)$. 
Table A-2: The machining parameters and the algorithm iterations for creating the semicircular protrusions in the brittle glass

\begin{tabular}{|c|c|c|c|c|c|c|c|c|c|c|c|c|c|c|c|c|c|c|c|c|c|c|c|c|c|c|c|}
\hline \multirow{2}{*}{$\begin{array}{c}\text { Featur } \\
\text { e }\end{array}$} & \multirow{2}{*}{ Parameter } & \multicolumn{26}{|c|}{ Source } \\
\hline & & 1 & 2 & 3 & 5 & & 5 & 7 & 8 & 9 & 10 & 11 & 12 & 13 & \begin{tabular}{|l|}
14 \\
\end{tabular} & 15 & 16 & 17 & 18 & 19 & 20 & 21 & 22 & 23 & 24 & 25 & 26 \\
\hline \multirow{4}{*}{ 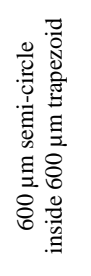 } & $\begin{array}{l}\text { Scan speed } \\
\left(v_{s}\right)_{K}(\mathbf{m m} / \mathbf{s})\end{array}$ & $\begin{array}{l}19 . \\
552\end{array}$ & $\begin{array}{l}10 . \\
618\end{array}$ & $\begin{array}{c}11 . \\
758\end{array}$ & $\begin{array}{l}11 . \\
855\end{array}$ & $\begin{array}{l}11 . \\
868\end{array}$ & $\begin{array}{l}11 . \\
889\end{array}$ & $\begin{array}{l}11 . \\
760\end{array}$ & $\begin{array}{l}11 . \\
714\end{array}$ & $\begin{array}{l}11 . \\
106\end{array}$ & $\begin{array}{l}18 . \\
231\end{array}$ & $\begin{array}{l}62 . \\
406\end{array}$ & $\begin{array}{l}62 . \\
406\end{array}$ & $\begin{array}{l}18 . \\
231\end{array}$ & $\begin{array}{l}11 . \\
106\end{array}$ & $\begin{array}{l}11 . \\
714\end{array}$ & $\begin{array}{l}11 . \\
760\end{array}$ & $\begin{array}{l}11 . \\
889\end{array}$ & $\begin{array}{l}11 . \\
868\end{array}$ & $\begin{array}{l}11 . \\
855\end{array}$ & $\begin{array}{l}11 . \\
758\end{array}$ & $\begin{array}{l}10 . \\
618\end{array}$ & $\begin{array}{l}19 . \\
522\end{array}$ & - & - & - & - \\
\hline & $\begin{array}{c}\begin{array}{c}\text { Offsets } \\
(\mu \mathrm{m})\end{array} \\
\end{array}$ & 0 & 208 & 416 & 620 & 821 & $\begin{array}{c}102 \\
0\end{array}$ & $\begin{array}{c}122 \\
3\end{array}$ & $\begin{array}{c}143 \\
0\end{array}$ & $\begin{array}{c}164 \\
2 \\
\end{array}$ & $\begin{array}{c}175 \\
3\end{array}$ & $\begin{array}{c}195 \\
0\end{array}$ & $\begin{array}{c}268 \\
6\end{array}$ & $\begin{array}{c}288 \\
4\end{array}$ & $\begin{array}{c}299 \\
5\end{array}$ & $\begin{array}{c}320 \\
6\end{array}$ & $\begin{array}{c}341 \\
3\end{array}$ & $\begin{array}{c}361 \\
6\end{array}$ & $\begin{array}{c}381 \\
6\end{array}$ & $\begin{array}{c}401 \\
6\end{array}$ & $\begin{array}{c}422 \\
1\end{array}$ & $\begin{array}{c}442 \\
9 \\
\end{array}$ & $\begin{array}{c}763 \\
6 \\
6\end{array}$ & - & - & - & - \\
\hline & $\begin{array}{c}\text { Mass flow } \\
\text { rate } \\
(\mathrm{g} / \mathrm{min})\end{array}$ & \multicolumn{26}{|c|}{3.6} \\
\hline & $\begin{array}{c}\text { Number of } \\
\text { repeats }\end{array}$ & \multicolumn{26}{|c|}{18} \\
\hline \multirow{4}{*}{ 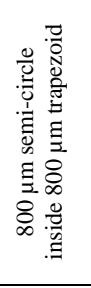 } & $\begin{array}{l}\text { Scan speed } \\
\left(v_{s}\right)_{K}(\mathbf{m m} / \mathbf{s})\end{array}$ & $\begin{array}{l}13 . \\
333\end{array}$ & $\begin{array}{l}7.2 \\
73\end{array}$ & $\begin{array}{l}9.4 \\
12\end{array}$ & $\begin{array}{l}8.8 \\
89\end{array}$ & $\begin{array}{l}8.8 \\
89\end{array}$ & $\begin{array}{l}8.8 \\
89\end{array}$ & $\begin{array}{l}8.8 \\
89\end{array}$ & $\begin{array}{l}25 . \\
000\end{array}$ & $\begin{array}{l}13 . \\
333\end{array}$ & $\begin{array}{l}6.1 \\
54\end{array}$ & $\begin{array}{l}17 . \\
778\end{array}$ & $\begin{array}{l}32 . \\
000\end{array}$ & $\begin{array}{c}107 \\
.23 \\
4\end{array}$ & $\begin{array}{c}107 \\
.23 \\
4\end{array}$ & $\begin{array}{l}32 . \\
000\end{array}$ & $\begin{array}{l}17 . \\
778\end{array}$ & $\begin{array}{l}6.1 \\
54\end{array}$ & $\begin{array}{l}13 . \\
333\end{array}$ & $\begin{array}{l}25 . \\
000\end{array}$ & $\begin{array}{l}8.8 \\
89\end{array}$ & $\begin{array}{l}8.8 \\
89\end{array}$ & $\begin{array}{l}8.8 \\
89\end{array}$ & $\begin{array}{l}8.8 \\
89\end{array}$ & $\begin{array}{l}9.4 \\
12\end{array}$ & $\begin{array}{l}7.2 \\
73\end{array}$ & $\begin{array}{l}13 . \\
333\end{array}$ \\
\hline & $\begin{array}{c}\text { Offsets } \\
(\mu \mathrm{m})\end{array}$ & 0 & 215 & 400 & 600 & 810 & $\begin{array}{c}101 \\
5\end{array}$ & $\begin{array}{c}122 \\
5\end{array}$ & $\begin{array}{c}140 \\
0\end{array}$ & $\begin{array}{c}145 \\
0\end{array}$ & $\begin{array}{c}165 \\
0\end{array}$ & $\begin{array}{c}180 \\
0\end{array}$ & $\begin{array}{c}195 \\
0\end{array}$ & $\begin{array}{c}215 \\
0\end{array}$ & $\begin{array}{c}285 \\
0\end{array}$ & $\begin{array}{c}305 \\
0\end{array}$ & $\begin{array}{c}320 \\
0\end{array}$ & $\begin{array}{c}335 \\
0\end{array}$ & $\begin{array}{c}355 \\
0\end{array}$ & $\begin{array}{c}360 \\
0\end{array}$ & $\begin{array}{c}377 \\
5\end{array}$ & $\begin{array}{c}398 \\
5\end{array}$ & $\begin{array}{c}419 \\
0\end{array}$ & $\begin{array}{c}440 \\
0\end{array}$ & $\begin{array}{c}460 \\
0\end{array}$ & $\begin{array}{c}478 \\
5\end{array}$ & $\begin{array}{c}500 \\
0\end{array}$ \\
\hline & $\begin{array}{c}\text { Mass flow } \\
\text { rate } \\
\text { (g/min) }\end{array}$ & \multicolumn{26}{|c|}{18.7} \\
\hline & $\begin{array}{c}\text { Number of } \\
\text { repeats }\end{array}$ & \multicolumn{26}{|c|}{15} \\
\hline \multirow{4}{*}{ 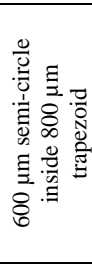 } & $\begin{array}{l}\text { Scan Speed } \\
\left(v_{s}\right)_{K}(\mathrm{~mm} / \mathrm{s})\end{array}$ & $\begin{array}{l}6.3 \\
71 \\
\end{array}$ & $\begin{array}{l}2.7 \\
63 \\
\end{array}$ & $\begin{array}{l}3.6 \\
22 \\
\end{array}$ & $\begin{array}{l}3.6 \\
05 \\
\end{array}$ & $\begin{array}{l}3.5 \\
98 \\
\end{array}$ & $\begin{array}{l}3.5 \\
69 \\
\end{array}$ & $\begin{array}{l}3.4 \\
99 \\
\end{array}$ & $\begin{array}{l}3.0 \\
59 \\
\end{array}$ & $\begin{array}{l}6.0 \\
62 \\
\end{array}$ & $\begin{array}{l}15 . \\
302 \\
\end{array}$ & $\begin{array}{l}10 . \\
137\end{array}$ & $\begin{array}{l}10 . \\
137\end{array}$ & $\begin{array}{r}15 . \\
302 \\
\end{array}$ & $\begin{array}{l}6.0 \\
62 \\
\end{array}$ & $\begin{array}{l}3.0 \\
59 \\
\end{array}$ & $\begin{array}{l}3.4 \\
99 \\
\end{array}$ & $\begin{array}{l}3.5 \\
69 \\
\end{array}$ & $\begin{array}{l}3.5 \\
98 \\
\end{array}$ & $\begin{array}{l}3.6 \\
05 \\
\end{array}$ & $\begin{array}{l}3.6 \\
22 \\
\end{array}$ & $\begin{array}{l}2.7 \\
63 \\
\end{array}$ & $\begin{array}{l}6.3 \\
71 \\
\end{array}$ & $\begin{array}{l}6.3 \\
71 \\
\end{array}$ & $\begin{array}{l}2.7 \\
63 \\
\end{array}$ & - & - \\
\hline & $\begin{array}{c}\text { Offsets } \\
(\mu \mathrm{m})\end{array}$ & 0 & 332 & 539 & 748 & 952 & $\begin{array}{c}115 \\
2 \\
\end{array}$ & $\begin{array}{c}135 \\
2 \\
\end{array}$ & $\begin{array}{c}155 \\
5 \\
\end{array}$ & $\begin{array}{c}176 \\
2 \\
\end{array}$ & $\begin{array}{c}197 \\
3 \\
\end{array}$ & $\begin{array}{c}208 \\
5 \\
\end{array}$ & $\begin{array}{c}208 \\
5 \\
\end{array}$ & $\begin{array}{c}228 \\
2 \\
\end{array}$ & $\begin{array}{c}301 \\
8 \\
\end{array}$ & $\begin{array}{c}321 \\
5 \\
\end{array}$ & $\begin{array}{c}321 \\
5 \\
\end{array}$ & $\begin{array}{c}332 \\
7 \\
\end{array}$ & $\begin{array}{c}353 \\
8 \\
\end{array}$ & $\begin{array}{c}374 \\
5 \\
\end{array}$ & $\begin{array}{c}394 \\
8 \\
\end{array}$ & $\begin{array}{c}414 \\
8 \\
\end{array}$ & $\begin{array}{c}434 \\
8 \\
\end{array}$ & $\begin{array}{c}455 \\
2 \\
\end{array}$ & $\begin{array}{c}476 \\
1 \\
\end{array}$ & - & - \\
\hline & $\begin{array}{c}\text { Mass flow } \\
\text { rate } \\
(\mathrm{g} / \mathrm{min})\end{array}$ & \multicolumn{26}{|c|}{7.8} \\
\hline & $\begin{array}{c}\text { Number of } \\
\text { repeats }\end{array}$ & \multicolumn{26}{|c|}{18} \\
\hline
\end{tabular}




\section{A.2. Table of Machining Parameters for Channels in PMMA}

Table A-3 lists the location of the nozzle and its scan speed at each point, the mass flow rate and with number of iterations through the inverse algorithm used to predict $E_{r}(x)$.

Table A-3: The machining parameters and the iterations through the inverse algorithm used to machine the specified channels in PMMA

\begin{tabular}{|c|c|c|c|c|c|c|c|c|c|c|c|}
\hline \multirow{2}{*}{ Feature } & \multirow{2}{*}{ Parameter } & \multicolumn{10}{|c|}{ Source } \\
\hline & & 1 & 2 & 3 & 4 & 5 & 6 & 7 & 8 & 9 & 10 \\
\hline \multirow{4}{*}{$\begin{array}{l}\text { Symmetric } \\
\text { Wedge }\end{array}$} & Scan Speed $\left(v_{s}\right)_{\kappa}(\mathrm{mm} / \mathrm{s})$ & $\begin{array}{c}13.33 \\
3\end{array}$ & $\begin{array}{c}66.66 \\
7\end{array}$ & 3.333 & $\begin{array}{c}66.66 \\
7\end{array}$ & $\begin{array}{c}13.33 \\
3\end{array}$ & - & - & - & - & - \\
\hline & Offsets $(\mu \mathrm{m})$ & 0 & 225 & 475 & 725 & 950 & & & & & \\
\hline & Mass flow rate $(\mathrm{g} / \mathrm{min})$ & \multicolumn{10}{|c|}{5.8} \\
\hline & $\begin{array}{l}\text { Number of Algorithm } \\
\text { Iterations }\end{array}$ & \multicolumn{10}{|c|}{65} \\
\hline \multirow{4}{*}{$\begin{array}{l}\text { Unsymetric } \\
\text { Wedge } 20^{\circ}\end{array}$} & Scan Speed $\left(v_{s}\right)_{\kappa}(\mathrm{mm} / \mathrm{s})$ & $\begin{array}{c}13.70 \\
4\end{array}$ & $\begin{array}{c}10.79 \\
7\end{array}$ & $\begin{array}{c}45.68 \\
2\end{array}$ & $\begin{array}{c}20.00 \\
0\end{array}$ & $\begin{array}{c}16.00 \\
0\end{array}$ & $\begin{array}{c}22.84 \\
1\end{array}$ & $\begin{array}{c}22.84 \\
1\end{array}$ & $\begin{array}{c}38.06 \\
8\end{array}$ & $\begin{array}{c}34.26 \\
1\end{array}$ & $\begin{array}{c}11.42 \\
0\end{array}$ \\
\hline & Offsets $(\mu \mathrm{m})$ & 0 & 300 & 490 & 570 & 710 & 775 & 950 & 975 & 1020 & 1100 \\
\hline & Mass flow rate $(\mathrm{g} / \mathrm{min})$ & \multicolumn{10}{|c|}{9.2} \\
\hline & $\begin{array}{c}\text { Number of Algorithm } \\
\text { Iterations }\end{array}$ & \multicolumn{10}{|c|}{73} \\
\hline \multirow{4}{*}{$\begin{array}{l}\text { Unsymetric } \\
\text { Wedge } 30^{\circ}\end{array}$} & Scan Speed $\left(v_{s}\right)_{k}(\mathrm{~mm} / \mathrm{s})$ & $\begin{array}{c}17.65 \\
0\end{array}$ & $\begin{array}{c}18.65 \\
9\end{array}$ & $\begin{array}{c}38.41 \\
5\end{array}$ & $\begin{array}{c}21.76 \\
9\end{array}$ & $\begin{array}{c}18.65 \\
9\end{array}$ & $\begin{array}{c}26.12 \\
2\end{array}$ & $\begin{array}{c}26.12 \\
2\end{array}$ & $\begin{array}{c}130.6 \\
11\end{array}$ & $\begin{array}{c}130.6 \\
11\end{array}$ & $\begin{array}{c}11.87 \\
4\end{array}$ \\
\hline & Offsets $(\mu \mathrm{m})$ & 0 & 275 & 400 & 575 & 680 & 850 & 940 & 1000 & 1025 & 1100 \\
\hline & Mass flow rate $(\mathrm{g} / \mathrm{min})$ & \multicolumn{10}{|c|}{15.2} \\
\hline & $\begin{array}{c}\text { Number of Algorithm } \\
\text { Iterations }\end{array}$ & \multicolumn{10}{|c|}{40} \\
\hline \multirow{4}{*}{$\begin{array}{l}\text { Unsymetric } \\
\text { Wedge } 45^{\circ}\end{array}$} & Scan Speed $\left(v_{s}\right)_{K}(\mathrm{~mm} / \mathrm{s})$ & 4.901 & 3.676 & 5.251 & 5.251 & 2.101 & 5.655 & 5.251 & 3.869 & - & - \\
\hline & Offsets $(\mu \mathrm{m})$ & 0 & 270 & 500 & 630 & 740 & 920 & 940 & 1020 & - & - \\
\hline & Mass flow rate ( $\mathrm{g} / \mathrm{min})$ & \multicolumn{10}{|c|}{4.4} \\
\hline & $\begin{array}{c}\text { Number of Algorithm } \\
\text { Iterations }\end{array}$ & \multicolumn{10}{|c|}{100} \\
\hline
\end{tabular}




\section{A.3. Second-Strike Calculations based on Ray-tracing and Rigid Body Dynamics}

The calculations presented in this section trace a particle and its contribution to erosion after ricocheting to a different location.

Figure A.1, traces a particle colliding with the instantaneous predicted surface at the $n$th repeat in coordinates $\left(x_{0}+r_{p} \cdot \sin \alpha, \xi\left(x_{0}+r_{p} \cdot \sin \alpha, n\right)+r_{p} \cdot \cos \alpha\right)$. This scenario assumes the particle to be spherical and accounts for its dimension to find the location of the second impact at coordinates $\left(x_{2 n d}+r_{p} \cdot \sin \mathrm{B}, \xi\left(x_{2 n d}+r_{p} \cdot \sin \mathrm{B}, n\right)+r_{p} \cdot \cos \mathrm{B}\right)$.

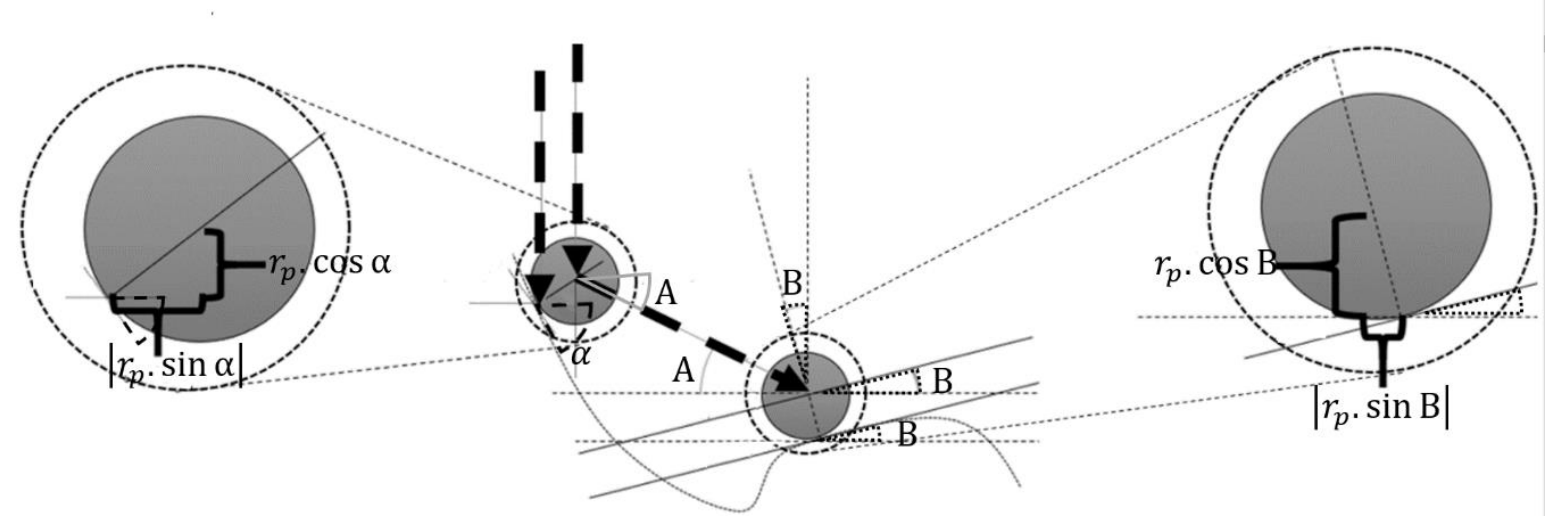

Figure A.1: Schematics of a ricocheting particle with the angles of interest with the dashed arrows showing the trajectory of points on the sphere.

According to the schematics above (Figure A.1), the trigonometric ratios for the angle $\alpha$ between the instantaneous surface and the horizon at the primary point of contact could be calculated, in any scenario as seen in set A-1:

$\left\{\begin{array}{l}\cos \alpha=\frac{1}{\sqrt{1+\left(\frac{\partial \xi\left(x_{0}+r_{p} \cdot \sin \alpha, n\right)}{\partial x}\right)^{2}}} \\ \sin \alpha=\frac{\frac{\partial \xi\left(x_{0}+r_{p} \cdot \sin \alpha, n\right)}{\partial x}}{\sqrt{1+\left(\frac{\partial \xi\left(x_{0}+r_{p} \cdot \sin \alpha, n\right)}{\partial x}\right)^{2}}}\end{array}\right.$

Also, $\tan \mathrm{A}$ is the slope of the second trajectory of the particle and $\tan \alpha$ is the slope of the tangent to the semicircular channel's profile at the point of impact. 
According to Brach [79], if the impulse ratio $\mu$ was below the critical impulse ratio $\mu_{c}$, the kinematics at the COM are dictated by set A-2:

$$
\left\{\begin{array}{c}
\bar{V}_{n}=-e \cdot v_{n} \\
\bar{V}_{t}=v_{t}-\mu(1+e) v_{n} \\
\Omega=\omega+2 \mu(1+e) \cdot \frac{v_{n}}{r_{p}}
\end{array}\right.
$$

where, $e$ is the coefficient of restitution, $\mu$ the coefficient of Coulomb friction which were extracted according to Slikkerveer and in't Veld's [23] previous findings for borosilicate glass and alumina (0.5 and 0.7). $v_{t}$ and $v_{n}$ are the initial tangential and normal components of velocity at COM before the impact and $\bar{V}_{t}$ and $\bar{V}_{n}$ the tangential and normal components of velocity at COM after the impact. The angular speed before the impact is $\omega($ assumed $01 / s)$ and that of after the impact is $\Omega$ and $r_{p}$ is the radius of the particle. The critical impulse ratio $\mu_{c}$, is given by $\mu_{c}=\frac{1}{1+\lambda} \cdot \frac{r}{1+e}[79]$. The last two coefficients, $\lambda$, and $r$, for a spherical particle would be reduced to [79]:

$$
\left\{\begin{array}{c}
\lambda=\frac{k_{G}^{2}}{r_{p}^{2}}=\frac{\left(\frac{r_{p}}{\sqrt{2}}\right)^{2}}{r_{p}^{2}}=\frac{1}{2} \\
r=\frac{v_{t}-r_{p} \cdot \omega}{v_{n}}=\frac{v_{t}}{v_{n}}=\frac{-\sin \alpha}{\cos \alpha}
\end{array}\right.
$$

where $k_{G}$ was the radius of gyration of the particle about its COM.

The change in momentum of the center of mass for a spherical particle is calculated by the impulse-momentum equation and combined with the definition of impulse ratio (which was the change in tangential over normal momentum) leading to Equation A-4 [79]:

$$
\frac{\left|\bar{V}_{t}-v_{t}\right|}{\left|-e . v_{n}-v_{n}\right|}=\mu
$$


By substituting the expression for each component of velocity, after the first strike "at the point of contact", one could rewrite the first two equations in set A-2 for a sphere as:

$$
\left\{\begin{array}{c}
\bar{V}_{n}=-e \cdot v\left(x_{0}\right) \cdot \cos \left(\tan ^{-1}\left(\frac{\partial \xi\left(x_{0}+r_{p} \cdot \sin \alpha, n\right)}{\partial x}\right)\right) \\
\bar{V}_{t}=v\left(x_{0}\right) \cdot \sin \left(\tan ^{-1}\left(\frac{\partial \xi\left(x_{0}+r_{p} \cdot \sin \alpha, n\right)}{\partial x}\right)\right)-\mu(1+e) \cdot v\left(x_{0}\right) \cdot \cos \left(\tan ^{-1}\left(\frac{\partial \xi\left(x_{0}+r_{p} \cdot \sin \alpha, n\right)}{\partial x}\right)\right)
\end{array}\right.
$$

where $v\left(x_{0}\right)$ was the original speed of the projected particle before impact.

The trajectory of the ricocheting particle can be found given its point of impact $\left(x_{0}+r_{p}\right.$. $\sin$ $\left.\alpha, \xi\left(x_{0}, n\right)+r_{p} \cdot \cos \alpha\right)$ and the slope of its trajectory, $\tan \mathrm{A}$, found by the equation set A-6:

$$
\left\{\begin{array}{c}
\tan \mathrm{A}=\frac{\Lambda+\tan (\alpha)}{1-\tan (\alpha)}=\frac{\Lambda+\frac{\partial \xi\left(x_{0}+r_{p} \cdot \sin \alpha\right)}{\partial x}}{1+\Lambda \cdot \frac{\partial \xi\left(x_{0}+r_{p} \cdot \sin \alpha\right)}{\partial x}} \\
\Lambda=\frac{-e \cdot v_{n}}{v_{t}-\mu(1+e) \cdot v_{n}} \rightarrow \Lambda=\frac{-e}{\tan \alpha-\mu(1+e)}=\frac{-e}{\frac{\partial \xi\left(x_{0}+r_{p} \cdot \sin \alpha\right)}{\partial x}-\mu(1+e)}
\end{array}\right.
$$

where $\Lambda$ is the ratio $\left(\frac{\bar{V}_{n}}{\bar{V}_{t}}\right)$.

The particle's size was taken into consideration when finding the trajectory of the particle's center of mass. With this information, $x_{2 n d}$, the lateral location of the particle's center of mass when second-strike occurs, can be found. This occurrence was identified if the trajectory of the ricocheting particle came closer than $r_{p}$ to the instantaneous surface $\xi(x, n)$, using the algorithm shown in Figure A.2.

If the distance abruptly reduced to a value below $r_{p}$, the meshes were not fine enough. in contrast, the distance would remain more than $r_{p}$ through the particle's trajectory if it never hit the surface. 


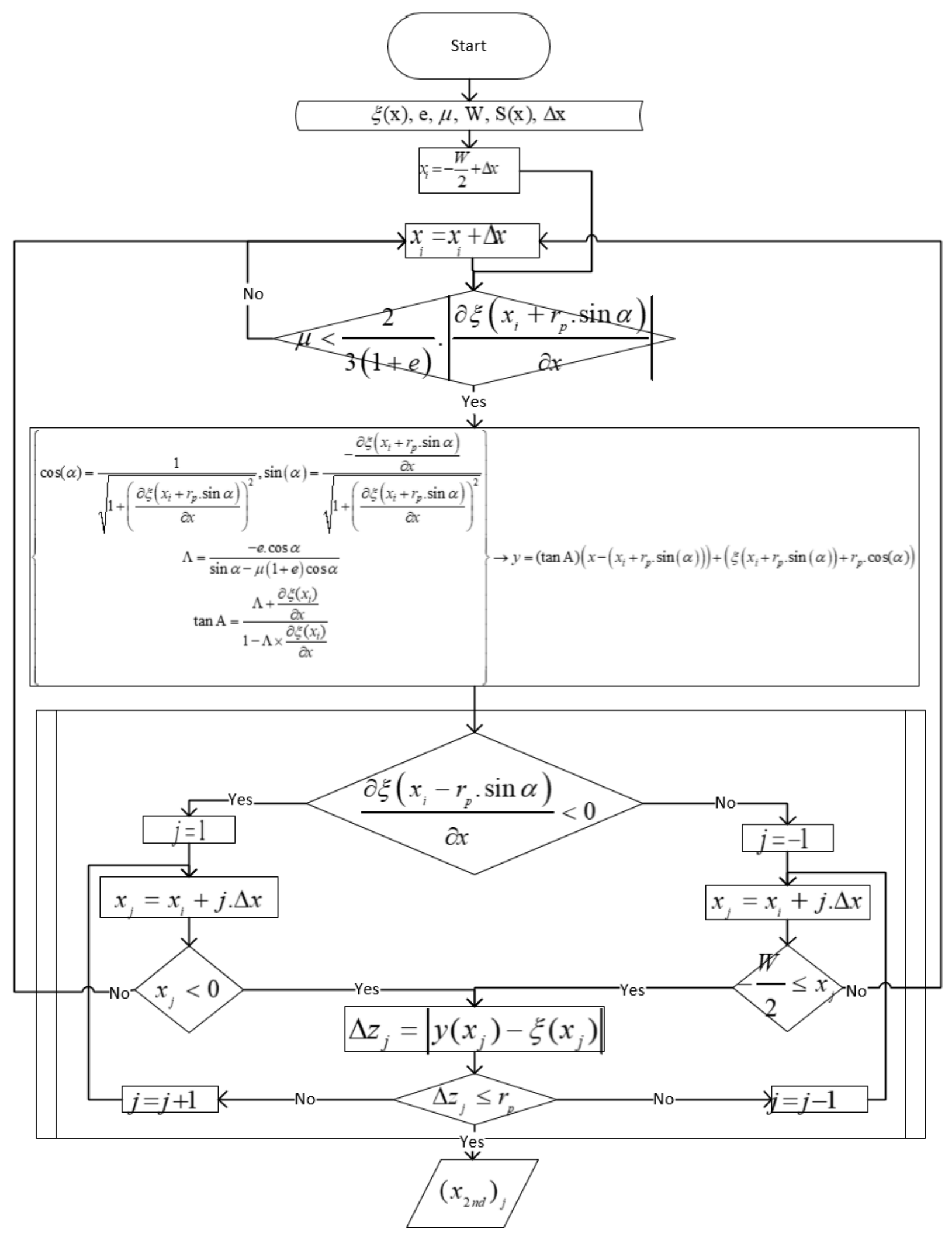

Figure A.2: The algorithm used to identify the location of second-strike 
To find the normal component of velocity at the point of second-strike, consider Figure A.1, in which the angle between normal to the instantaneous surface and the second-strike's projectile would be $\frac{\pi}{2}-(B-A)$. Thus, if the speed of the center of mass at second-strike was denoted by $\bar{V}$, one could write the normal component of velocity at the second-strike location with $-\bar{V} \cdot \cos \left(\frac{\pi}{2}+\mathrm{A}-\mathrm{B}\right)=-\bar{V} \cdot \sin (\mathrm{B}-\mathrm{A})$, and, the angles $\mathrm{A}$ and $\mathrm{B}$ could be found by A-7:

$$
\left\{\begin{array}{c}
\mathrm{B}=\tan ^{-1}\left(\frac{\partial \xi\left(x_{2 n d}+r_{p} \cdot \sin \mathrm{B}, n\right)}{\partial x}\right) \\
\tan ^{-1}\left(\frac{\bar{V}_{n}}{\bar{V}_{t}}\right)=\mathrm{A}-\alpha \rightarrow \mathrm{A}=\alpha+\tan ^{-1}\left(\frac{\bar{V}_{n}}{\bar{V}_{t}}\right)=\alpha+\tan ^{-1}\left(\frac{-e}{\frac{\partial \xi\left(x_{0}+r_{p} \cdot \sin \alpha\right)}{\partial x}-\mu(1+e)}\right) \mathrm{A}-7
\end{array}\right.
$$

Given the equation 2-3 and the superposed erosive efficacy at the first location of impact $S\left(x_{0}\right)$ one can find the oblique contribution to erosion at $\left(x_{2 n d}+r_{p} \cdot \sin \mathrm{B}, \xi\left(x_{2 n d}+r_{p} \cdot \sin \mathrm{B}, n\right)+r_{p} \cdot \cos \right.$ B) which can be projected along the vertical, yielding Equation A-8:

$$
E_{2 n d}\left(x_{2 n d}+r_{p} \cdot \sin \mathrm{B}, n\right)=\left(\frac{\bar{V}}{v\left(x_{0}\right)}\right)^{k} \cdot \sin (\mathrm{B}-\mathrm{A}) \cdot \cos \mathrm{B} \cdot S\left(x_{0}\right)
$$

Through every repeat, this second-strike contribution along the normal was calculated and added to find the final contribution of second-strike to erosion.

If sliding does not occur, the kinetic equations in set A-2 become:

$$
\left\{\begin{array}{c}
\bar{V}_{n}=-e \cdot v_{n} \\
\bar{V}_{t}=r_{p} \cdot \Omega \\
\Omega=\omega+\frac{v\left(x_{0}\right) \cdot \sin \alpha}{r_{p}}
\end{array}\right.
$$

However, such a particle would not travel far enough for the possibility of its erosion at a location other than where it primarily struck the surface to be significant. 


\section{References:}

[1] Y.-J. Chiu, C. Cho Sung Hwan, Z. Mei, V. Lien, T.-F. Wu, and L. Lo Yu-Hwa, "Universally applicable three-dimensional hydrodynamic microfluidic flow focusing," Lab. Chip, vol. 13, no. 9, pp. 183-189, Apr. 2013.

[2] H. Y. Wu and P. Cheng, "An experimental study of convective heat transfer in silicon microchannels with different surface conditions," Int. J. Heat Mass Transf., vol. 46, pp. 2547-2556, 2003.

[3] G. Kucukturk and C. Cogun, "A New Method for Machining of Electrically Nonconductive Workpieces Using Electric Discharge Machining,” Mach. Sci. Technol., vol. 14, no. 2, pp. 189-207, Aug. 2010.

[4] H. A. G. El-Hofy, Advanced Machining Processes: Nontraditional and Hybrid Machining Processes. McGraw Hill Professional, 2005, 2005.

[5] Sookhak Lari, M.R.; Papini, M., "Inverse methods to gradient etch three-dimensional features with prescribed topographies using abrasive jet micro-machining: Part I - Modelling," Precis. Eng., vol. 45, pp. 272-284, 2016.

[6] D. Axinte, J. Billingham, and A. B. Guillerna, "New models for energy beam machining enable accurate generation of free forms," Sci. Adv., pp. 1-8, 2017.

[7] K. . van Dijke, G. Veldhuis, C. G. P. . Schroën, and R. . Boom, "Parallelized edge-based droplet generation (EDGE) devices," Lab. Chip, vol. 9, no. 19, pp. 2824-2830, 2009.

[8] Y. Jun et al., "Microfluidics-generated pancreatic islet microfibers for enhanced immunoprotection," Biomaterials, vol. 34, no. 33, pp. 8122-8130, 2013.

[9] M. Roederer, J. M. Brenchley, M. R. Betts, and S. C. De Rosa, "Flow cytometric analysis of vaccine responses: How many colors are enough?," Clin. Immunol., vol. 110, no. 3, pp. 199-205, 2004.

[10] C. D. Jennings and K. A. Foon, "Flow cytometry: recent advances in diagnosis and monitoring leukemia," Cancer Invest., vol. 15, no. 4, pp. 384-399, 1997.

[11] A. K. Lehmann, S. Sørnes, and A. Halstensen, "Phagocytosis: Measurement by flow cytometry," $J$. Immunol. Methods, vol. 243, no. 1, pp. 229-242, 2000.

[12] M. Keeney, I. Chin-Yee, K. Weir, J. Popma, R. Nayar, and D. R. Sutherland, "Single platform flow cytometric absolute CD34+ cell counts based on the ISHAGE guidelines," Cytometry, vol. 34, no. 2, pp. 61-70, Apr. 1998.

[13] C. Iliescu, B. Chen, and J. Miao, "On the wet etching of Pyrex glass," Sens. Actuators Phys., vol. 143, no. 1, pp. 154-161, 2008.

[14] X. Cheng, Z. Zhigang, K. Nakamoto, and K. Yamazaki, "A study on the micro tooling for micro/nano milling,” Int. J. Adv. Manuf. Technol., vol. 53, no. 5, pp. 523-533, Mar. 2011.

[15] H. T. Liu, Y. Z. Sun, D. B. Shan, and C. X. Zhang, "Experimental research of brittle-ductile transition conditions and tool wear for micromilling of glass material," Int. J. Adv. Manuf. Technol., vol. 68, no. 5, pp. 1901-1909, Sep. 2013.

[16] Z.-P. Zheng, J.-K. Lin, F.-Y. Huang, and B.-H. Yan, "Improving the machining efficiency in electrochemical discharge machining (ECDM) microhole drilling by offset pulse voltage," $J$. Micromechanics Microengineering, vol. 18, no. 2, pp. 1-6, Feb. 2008.

[17] H. Ogura and Y. Yoshida, "Hole Drilling of Glass Substrates with a $\mathrm{CO}_{2}$ Laser," Jpn. J. Appl. Phys., vol. 42, no. 5, p. 2881, May 2003.

[18] A. Sayah, P.-A. Thivolle, V. K. Parashar, and M. A. M. Gijs, "Fabrication of microfluidic mixers with varying topography in glass using the powder-blasting process," J. Micromechanics Microengineering, vol. 19, p. 085024, Aug. 2009.

[19] J. R. Anderson et al., "Fabrication of topologically complex three-dimensional microfluidic systems in PDMS by rapid prototyping," Anal. Chem., vol. 72, no. 14, pp. 3158-3164, Jul. 2000. 
[20] A. Ghobeity, T. Krajac, T. Burzynski, M. Papini, and J. K. Spelt, "Surface evolution models in abrasive jet micromachining," Wear, vol. 264, no. 3-4, pp. 185-198, Feb. 2008.

[21] E. Belloy, A.-G. Pawlowski, A. Sayah, and M. A. M. Gijs, "Microfabrication of high-aspect ratio and complex monolithic structures in glass," J. Microelectromechanical Syst., vol. 11, no. 5, pp. 521-527, 2002.

[22] R. Haj Mohammad Jafar, J. K. Spelt, and M. Papini, "Surface roughness and erosion rate of abrasive jet micro-machined channels: Experiments and analytical model," Wear, vol. 303, no. 1-2, pp. 138-145, Jun. 2013.

[23] P. J. Slikkerveer and F. int Veld, "Model for patterned erosion," Wear, vol. 233, pp. 377-386, 1999.

[24] H. Z. Li and J. M. Fan, "Analysis and modelling of particle velocities in micro-abrasive air jet," Int. J. Mach. Tools Manuf., vol. 49, pp. 850-858.

[25] I. Finnie, "Erosion of surfaces by solid particles," Wear, vol. 3, no. 2, pp. 87-103, 1960.

[26] D. . MARSHALL, B. . LAWN, and A. . EVANS, "Elastic/Plastic Indentation Damage in Ceramics: The Lateral Crack System," J. Am. Ceram. Soc., vol. 65, no. 11, pp. 561-566, Nov. 1982.

[27] A. Ghobeity, D. Ciampini, and M. Papini, "An analytical model of the effect of particle size distribution on the surface profile evolution in abrasive jet micromachining," J. Mater. Process. Technol., vol. 209, no. 20, pp. 6067-6077, Nov. 2009.

[28] J. Y. Sheikh-Ahmad, Machining of Polymer Composites. Springer, 2009.

[29] H. Getu, J. K. Spelt, and M. Papini, "Conditions leading to the embedding of angular and spherical particles during the solid erosion of polymers," Wear, vol. 292-293, pp. 159-168, Jul. 2012.

[30] G. P. Tilly and W. Sage, "The interaction of particle and material behaviour in erosion processes," Wear, vol. 16, no. 6, pp. 447-465, Dec. 1970.

[31] I. Finnie, "Some observations on the erosion of ductile metals," Wear, vol. 19, no. 1, pp. 81-90, 1972.

[32] G. . Tilly, "A two stage mechanism of ductile erosion," Wear, vol. 23, no. 1, pp. 87-96, 1973.

[33] I. . Hutchings and R. . Winter, "Particle erosion of ductile metals: A mechanism of material removal," Wear, vol. 27, no. 1, pp. 121-128, 1974.

[34] P. J. Slikkerveer, P. C. P. Bouten, and H. Scholten, "Erosion and damage by sharp particles," Wear, vol. 217, no. 2, pp. 237-250, 1998.

[35] Y. I. Oka, H. Ohnogi, T. Hosokawa, and M. Matsumura, "The impact angle dependence of erosion damage caused by solid particle impact," Wear, vol. 203-204, pp. 573-579, Mar. 1997.

[36] G. L. Sheldon and I. Finnie, "On the Ductile Behavior of Nominally Brittle Materials During Erosive Cutting," J. Eng. Ind., vol. 88, no. 4, pp. 387-392, Nov. 1966.

[37] A. . Gradeen, M. Papini, and J. K. Spelt, "The effect of temperature on the cryogenic abrasive jet micro-machining of polytetrafluoroethylene," Wear, vol. 317, no. 1-2, pp. 170-178, Sep. 2014.

[38] J. Takadoum and H. Houmid Bennani, "Influence of substrate roughness and coating thickness on adhesion," Surf. Coat. Technol., vol. 96, pp. 272-282, 1997.

[39] H. Yandong, C. Werner, and D. Li, "Influence of Three-Dimensional Roughness on PressureDriven Flow Through Microchannels," J. Fluids Eng., vol. 125, no. 5, pp. 871-879, Oct. 2003.

[40] I. M. Hutchings, "Ductile-brittle transitions and wear maps for the erosion and abrasion of brittle materials," J. Phys. Appl. Phys., vol. 25, p. A212, 1992.

[41] M. Papini and S. Dhar, "Experimental verification of a model of erosion due to the impact of rigid single angular particles on fully plastic targets," Int. J. Mech. Sci., vol. 48, no. 5, pp. 469-482, 2006.

[42] R. H. M. Jafar, J. K. Spelt, and M. Papini, "Numerical simulation of surface roughness and erosion rate of abrasive jet micro-machined channels," Wear, vol. 303, no. 1-2, pp. 302-312, Jun. 2013.

[43] H. Wensink, S. Schlautmann, M. H. Goedbloed, and M. C. Elwenspoek, "Fine tuning the roughness of powder blasted surfaces," J. Micromechanics Microengineering, vol. 12, no. 5, pp. 616-620, 2002.

[44] R. H. M. Jafar, J. K. Spelt, and M. Papini, "Surface Finishing of Micro-channels Using Low Kinetic Energy Abrasives,” Int. J. Mech. Eng. Mechatron., vol. 2, pp. 43-50, 2014. 
[45] T. Burzynski and M. Papini, "Analytical model of particle interference effects in divergent erosive jets," Tribol. Int., vol. 43, no. 3, pp. 554-567, 2010.

[46] L. Zhang, T. Kuriyagawa, Y. Yasutomi, and J. Zhao, "Investigation into micro abrasive intermittent jet machining,” Int. J. Mach. Tools Manuf., vol. 45, no. 7-8, pp. 873-879, 2005.

[47] H. Getu, A. Ghobeity, J. K. Spelt, and M. Papini, "Abrasive jet micromachining of polymethylmethacrylate," Wear, vol. 263, no. 7-12, pp. 1008-1015, Sep. 2007.

[48] A. Noohi, M. R. Sookhak Lari, J. K. Spelt, and M. Papini, "Implementation of a shadow mask for direct writing in abrasive jet micro-machining," J. Mater. Process. Technol., vol. 223, pp. 232-239, 2015.

[49] A. Ghobeity, M. Papini, and J. K. Spelt, "Computer simulation of particle interference in abrasive jet micromachining," Wear, vol. 263, no. 1-6, pp. 265-269, Sep. 2007.

[50] D. Solignac, A. Sayah, S. Constantin, R. Freitag, and M. A. M. Gijs, "Powder blasting for the realisation of microchips for bio-analytic applications," Sens. Actuators Phys., vol. 92, no. 1-3, pp. 388-393, 2001.

[51] A. Ghobeity, H. J. Crabtree, M. Papini, and J. K. Spelt, "Characterisation and comparison of microfluidic chips formed using abrasive jet micromachining and wet etching," J. Micromechanics Microengineering, vol. 22, no. 2, p. 025014, Feb. 2012.

[52] K. Pang, J. Wang, and T. Nguyen, "Mechanisms of microhole formation on glasses by an abrasive slurry jet," J. Appl. Phys., vol. 105, no. 4, pp. 044906-044906-4, Feb. 2009.

[53] M. R. Sookhak Lari, A. Ghazavi, and M. Papini, "A rotating mask system for sculpting of threedimensional features using abrasive jet micro-machining," J. Mater. Process. Technol., vol. 243, pp. 62-74, May 2017.

[54] Sharma, D.; Singh, P.P.; Garg, H., "Comparative study of rectangular and trapezoidal microchannels using water and liquid metal," Procedia Eng., vol. 51, pp. 791-796, 2013.

[55] D. S. Park, M. W. Cho, H. Lee, and W. S. Cho, "Micro-grooving of glass using micro-abrasive jet machining," J. Mater. Process. Tech, vol. 146, no. 2, pp. 234-240, 2004.

[56] A. Ghobeity, T. Krajac, T. Burzynski, M. Papini, and J. K. Spelt, "Surface evolution models in abrasive jet micromachining," Wear, vol. 264, no. 3-4, pp. 185-198, Feb. 2008.

[57] M. R. Sookhak Lari and M. Papini, "Inverse methods to gradient etch three-dimensional features with prescribed topographies using abrasive jet micro-machining: Part I - Modeling," Precis. Eng., vol. 45, pp. 272-284, Mar. 2016.

[58] D. R. Andrews, "An analysis of solid particle erosion mechanisms," J Phys Appl Phys, vol. 14, no. 11, pp. 1979-1991, Nov. 1981.

[59] D. S. Park, M. W. Cho, and H. Lee, "Effects of the impact angle variations on the erosion rate of glass in powder blasting process," Int. J. Adv. Manuf. Technol., vol. 23, no. 5, pp. 444-450, 2004.

[60] D. B. Marshall, A. G. Evans, M. E. Gulden, J. L. Routbort, and R. O. Scattergood, "Particle size distribution effects on the solid particle erosion of brittle materials," Wear, vol. 71, no. 3, pp. 363373, 1981.

[61] I. Finnie and D. H. McFadden, "On the velocity dependence of the erosion of ductile metals by solid particles at low angles of incidence," Wear, vol. 48, no. 1, pp. 181-190, 1978.

[62] J. Bitter, "A study of erosion phenomena part I," Wear, vol. 6, no. 1, pp. 5-21, 1963.

[63] I. Finnie, G. R. Stevick, and J. R. Ridgely, "The influence of impingement angle on the erosion of ductile metals by angular abrasive particles," Wear, vol. 152, no. 1, pp. 91-98, 1992.

[64] J. H. M. ten Thije Boonkkamp and J. K. M. Jansen, "An analytical solution for mechanical etching of glass by powder blasting,” J. Eng. Math., vol. 43, no. 2, pp. 385-399, 2002.

[65] H. Getu, A. Ghobeity, J. K. Spelt, and M. Papini, "Abrasive jet micromachining of acrylic and polycarbonate polymers at oblique angles of attack," Wear, vol. 265, no. 5-6, pp. 888-901, Aug. 2008.

[66] S. Ally, J. K. Spelt, and M. Papini, "Prediction of machined surface evolution in the abrasive jet micro-machining of metals," Wear, vol. 292-293, pp. 89-99, Jul. 2012. 
[67] P. J. Slikkerveer, P. C. P. Bouten, and H. Scholten, "Erosion and damage by sharp particles," Wear, vol. 217, no. 2, pp. 237-250, 1998.

[68] A. Ghobeity, D. Ciampini, and M. Papini, "An analytical model of the effect of particle size distribution on the surface profile evolution in abrasive jet micromachining," J. Mater. Process. Technol., vol. 209, no. 20, pp. 6067-6077, Nov. 2009.

[69] T. Burzynski and M. Papini, "Level set methods for the modelling of surface evolution in the abrasive jet micromachining of features used in MEMS and microfluidic devices," $J$. Micromechanics Microengineering, vol. 20, no. 8, p. 085004, Aug. 2010.

[70] D. Ciampini and M. Papini, "A cellular automata and particle-tracking simulation of abrasive jet micromachining that accounts for particle spatial hindering and second strikes," J. Micromechanics Microengineering, vol. 20, no. 4, p. 045025, Apr. 2010.

[71] A. Ghobeity, M. Papini, and J. K. Spelt, "Abrasive jet micro-machining of planar areas and transitional slopes in glass using target oscillation," J. Mater. Process. Technol., vol. 209, no. 11, pp. 5123-5132, Jun. 2009.

[72] M. R. Sookhak Lari, A. Ghazavi, and M. Papini, "A rotating mask system for sculpting of threedimensional features using abrasive jet micro-machining," J. Mater. Process. Technol., vol. 243, pp. 62-74, May 2017.

[73] J. Billingham, C. B. Miron, D. A. Axinte, and M. C. Kong, "Mathematical modelling of abrasive waterjet footprints for arbitrarily moving jets: Part II-Overlapped single and multiple straight paths," Int. J. Mach. Tools Manuf., vol. 68, pp. 30-39, May 2013.

[74] N. Tamannaee, J. K. Spelt, and M. Papini, "Abrasive slurry jet micro-machining of edges, planar areas and transitional slopes in a talc-filled co-polymer," Precis. Eng., pp. 52-62, 2016.

[75] R. D. Ja“" ggi, R. Sandoz, and C. S. Effenhauser, "Microfluidic depletion of red blood cells from whole blood in high-aspect-ratio microchannels," Microfluid Nanofluid, vol. 3, pp. 47-53, 2007.

[76] A. Ghobeity, H. Getu, M. Papini, and J. K. Spelt, "Surface evolution models for abrasive jet micromachining of holes in glass and polymethylmethacrylate (PMMA)," J. Micromechanics Microengineering, vol. 17, no. 11, pp. 2175-2185, Nov. 2007.

[77] A. Ghobeity, H. Getu, T. Krajac, J. K. Spelt, and M. Papini, "Process repeatability in abrasive jet micro-machining," J. Mater. Process. Technol., vol. 190, no. 1-3, pp. 51-60, Jul. 2007.

[78] L. S. Lasdon, R. L. Fox, and M. W. Ratner, "Nonlinear optimization using generalized reduced gradient method," Rev. Fr. Autom. Inform. Rech. Opérationnelle, vol. 3, no. 8, pp. 73-103, 1974.

[79] R. M. Brach, "Formulation of rigid body impact problems using generalized coefficients," Int. J. Eng. Sci., vol. 36, no. 1, pp. 61-71, Jan. 1998. 